COMMUNICATIONS IN

ANALYSIS AND GEOMETRY

Volume 4, Number 3, 339-413, 1996

\title{
Perturbation Series for the Schrödinger Operator with a Periodic Potential near Planes of Diffraction
}

\author{
YU. E. KARPESHINA
}

\begin{abstract}
Perturbation series for Bloch eigenvalues and their spectral projections in a vicinity of von Laue diffraction planes are constructed. They are converging series with respect to a model operator, roughly taking into account the diffraction inside a crystal. The behavior of the isoenergetic surface near von Laue diffraction planes is described.
\end{abstract}

\section{Introduction.}

The motion of a particle in a bulk matter is often described by the Schrödinger operator with a periodic potential. Therefore, it is interesting to have a detailed mathematical analysis of its spectral properties, in particular, to construct perturbation formulae for its eigenvalues and eigenfunctions.

Thus, we consider the operator

$$
H=-\Delta+V
$$

in $L_{2}\left(R^{3}\right)$, where $V$ is the operator of multiplication by a periodic potential, a trigonometric polynomial.

In [1] and [2], perturbation series for Bloch eigenvalues and spectral projections were constructed on a rich set of quasimomenta. On this set, the perturbed and unperturbed eigenvalues and spectral projectors turn out to be close. Our aim here is to study the case when there exists a real refraction in the crystal, i.e., the perturbation is significant. Namely, we construct perturbation formulae for quasimomenta in a vicinity of the Laue diffraction planes. We describe Bloch eigenvalues and their spectral projections of $H$, which are close to those of some model operator, roughly taking into account the refraction inside the crystal.

To simplify notation, we consider the case where the cell of periods is orthogonal. All arguments go through, with obvious modifications, for a 
nonorthogonal lattice. We use a representation of the potential $V(x)$ in the form :

$$
V(x)=\sum_{m \in Z^{3},|m|<R_{0}} v_{m} \exp i\left(\vec{p}_{m}(0), x\right),
$$

where $0<R_{0}<\infty,(\cdot, \cdot)$ is the inner product in $R^{3}$ and $\vec{p}_{m}(0)$ is a vector of the dual lattice:

$$
\vec{p}_{m}(0)=2 \pi\left(m_{1} a_{1}^{-1}, m_{2} a_{2}^{-1}, m_{3} a_{3}^{-1}\right) .
$$

The potential $V$ is real, so $v_{m}=\bar{v}_{-m}$. It may be assumed with no loss of generality that $v_{0}=0$.

I. M. Gelfand [3] showed that the investigation of the spectral properties of $H$ reduces to the study of a family of regular operators $H(t), t \in K$, where $K$ is the elementary cell of the dual lattice,

$$
K=\left[0,2 \pi a_{1}^{-1}\right) \times\left[0,2 \pi a_{2}^{-1}\right) \times\left[0,2 \pi a_{3}^{-1}\right) .
$$

The vector $t$ is called the quasimomentum. Let $Q$ be the elementary cell of the periods of the potential, $Q=\left[0, a_{1}\right) \times\left[0, a_{2}\right) \times\left[0, a_{3}\right)$. The operator $H(t), t \in K$ acts in $L_{2}(Q)$. It is described by formula (1.1) and the quasiperiodic conditions:

$$
\begin{aligned}
& u\left(a_{1}, x_{2}, x_{3}\right)=\exp \left(i t_{1} a_{1}\right) u\left(0, x_{2}, x_{3}\right), \\
& u\left(x_{1}, a_{2}, x_{3}\right)=\exp \left(i t_{2} a_{2}\right) u\left(x_{1}, 0, x_{3}\right), \\
& u\left(x_{1}, x_{2}, a_{3}\right)=\exp \left(i t_{3} a_{3}\right) u\left(x_{1}, x_{2}, 0\right) .
\end{aligned}
$$

The first derivatives with respect to $x_{j}, j=1,2,3$ must also satisfy similar conditions.

The operator $H(t)$ has a discrete, bounded below spectrum $\Lambda(t)$ :

$$
\Lambda(t)=\cup_{n=1}^{\infty} \lambda_{n}(t), \lambda_{n}(t) \rightarrow_{n \rightarrow \infty} \infty .
$$

The spectrum $\Lambda$ of the operator $H$ is the union of the spectra of the operators $H(t)$ :

$$
\Lambda=\cup_{t \in K} \Lambda(t)=\cup_{n \in N, t \in K} \lambda_{n}(t) .
$$


The functions $\lambda_{n}(t)$ are continuous, so $\Lambda$ has a band structure:

$$
\Lambda=\cup_{n=1}^{\infty}\left[q_{n}, Q_{n}\right], q_{n}=\min _{t \in K} \lambda_{n}(t), Q_{n}=\max _{t \in K} \lambda_{n}(t) .
$$

The eigenfunctions of operator $H$ are obtained by quasiperiodic continuation (see (1.3)) of the eigenfunctions of all the operators $H(t)$ to $R^{n}$.

Let $H_{0}(t)$ be the operator corresponding to the zero potential. Its eigenfunctions are the plane waves:

$$
\exp \left(i\left(\vec{p}_{j}(t), x\right)\right), j \in Z^{3}, \vec{p}_{j}(t)=\vec{p}_{j}(0)+t .
$$

The eigenfunction (1.4) corresponds to the eigenvalue $p_{j}^{2}(t) \equiv\left|\vec{p}_{j}(t)\right|^{2}$. Thus, the spectrum of the free operator $H_{0}$ is:

$$
\Lambda_{0}(t)=\left\{p_{j}^{2}(t)\right\}_{j \in Z^{3}}
$$

Using the basis (1.4) we write the matrix of $H(t)$ in the form:

$$
H(t)_{m j}=p_{m}^{2}(t) \delta_{m j}+v_{m-j},
$$

where $\delta_{m j}$ is the Kronecker symbol.

The important problem is to construct perturbation formulae for eigenvalues and spectral projections of $H(t)$ in a high energy region. To explain the difficulties of this problem let us consider the eigenvalues $p_{m}^{2}(t)$ when $m \rightarrow \infty$. It is easy to see that the average distance between them goes to zero. In fact, let us consider $p_{m}^{2}(t)$ in the interval $\left((k-c)^{2},(k+c)^{2}\right)$, when $k \rightarrow \infty, c$ is a constant. The average distance $d$ is given by $d=2 c k / N$, where $2 c k$ is the length of the interval, $N$ is the number of the eigenvalues inside the interval. It is easy to see that $p_{m}^{2}(t)$ is in the interval if and only if $\vec{p}_{m}(t)$ is in the spherical shell $\{k-c<|x|<k+c\}$. Using the fact that $\vec{p}_{m}(t)$ are points of the dual lattice, it is not hard to show that $N$ is of order of the volume of the spherical shell, i.e., $N \approx c_{1} k^{2}$. Thus, $d \approx c_{2} k^{-1}$. The last relation means that the average distance between $p_{m}^{2}(t)$ goes to zero when $|m| \rightarrow \infty$. So, under a perturbation the eigenvalues influence each other strongly. To describe the perturbation of one of them one has to consider the perturbations of its neighbors. It turns out that under perturbation the eigenvalues show a different behavior. So do the corresponding spectral projections. Most of them are stable in the sense that their perturbations are asymptotically small when $|m| \rightarrow \infty$ (see [1], [2]). However, there are 
eigenvalues, which are strongly influenced by the perturbation, and so are their spectral projections. We shall study the latter situation here.

Naturally, the type of behavior of an eigenvalue is determined by $m, t$. For physical applications it is important to consider $m, t$ such that $p_{m}^{2}(t)=$ $k^{2}, k^{2}$ being fixed. According to this, in the physical literature the important concept of the isoenergetic surface $S_{0}(k)$ of the free operator is used (see f.e. [4]). This is the set of $t$ in $K$, for which the free operator $H_{0}(t)$ has an eigenvalue equal to $k^{2}$. It is obvious that $t \in S_{0}(k)$ if and only if the relation $p_{m}^{2}(t)=k^{2}$ for some $m \in Z^{3}$. The set $S_{0}(k)$ is obtained by partitioning the sphere of radius $k$ centered at zero into pieces by the dual lattice. After this the pieces are translated in a parallel manner into $K$.

Let $S_{1}(k)$ be a subset of $S_{0}(k)$. We say that $S_{1}(k)$ has an asymptotically full measure on $S_{0}(k)$ as $k \rightarrow \infty$ if

$$
\frac{s\left(S_{1}(k)\right)}{s\left(S_{0}(k)\right)} \rightarrow_{k \rightarrow \infty} 1
$$

here and below $s(\cdot)$ is the surface area.

In [1] a nonsingular set $S(k, V, \delta) \subset S_{0}(k)$ was constructed, so that for any point $t$ of the $\left(k^{-2-2 \delta}\right)$-neighborhood of it, the perturbed and unperturbed spectral projections and eigenfunctions are close - more precisely, the corresponding perturbation series converge. The nonsingular set has an asymptotically full measure on $S_{0}(k)$ (from this it easily follows that most of the eigenvalues $p_{m}^{2}(t)$ are stable). However, besides the nonsingular set, there exists a part of $S_{0}(k)$, where perturbed and unperturbed eigenvalues and eigenfunctions are, generally speaking, not close. We call it the singular set. To explane the nature of this set let us consider the plane wave $\exp i\left(\vec{p}_{j}(t), x\right)$, such that its momentum $\vec{p}_{j}(t)$ satisfies the von Laue diffraction conditions, i.e., $p_{j}(t)=p_{j+q}(t)$ for some $q \neq 0$. It is wellknown [4] that this wave is refracted by the crystal, the refracted wave being $a \exp i\left(\vec{p}_{j+q}(t), x\right), a \in R$. The latter wave interferes with the initial one and distorts it strongly. Hence, perturbed and unperturbed eigenfunctions obviously are not close, when $t$ satisfies the von Laue diffraction conditions. Thus, the existence of the singular set is connected with diffraction conditions - in the simplest situation with the von Laue diffraction conditions.

The unstable case was studied by J. Feldman, H. Knörrer, E. Trubowitz [5] in the two and three dimensional situations. In the three dimensional case they study the eigenvalues of $H$, which are not close to the unperturbed ones, but can be approximated by eigenvalues of the operator $-\Delta+V_{\gamma}$, where $\gamma$ some vector of the dual lattice and $V_{\gamma}$ is independent of $x$ in the direction 
$\gamma$ i.e.,

$$
V_{\gamma}(x)=V_{\gamma}\left(x-\gamma(x, \gamma)|\gamma|^{-2}\right)=\sum_{j:\left(\vec{p}_{j}(0), \gamma\right)=0} v_{j} \exp i\left(x, \vec{p}_{j}(0)\right) .
$$

It was proved that for arbitrary $\gamma$ of the dual lattice and any eigenvalue of $H_{\gamma}(t)$ corresponding to a sufficiently large momentum in the direction $\gamma$, there exists a close eigenvalue of the operator $H(t)$ with the same quasimomentum, multiplicity being taken into account. The same result was proved for $n=2$. Moreover, in the two-dimensional case it was shown that on the rich set of $t$ the corresponding eigenfunctions are close, too. O.A. Veliev discussed this problem [6].

We use another approach here, which has its own peculiarities. It provides formulae not only for unstable eigenvalues, but also for their spectral projections in the three-dimensional situation. Converging perturbation series, with respect to some model operator roughly describing the refraction, are constructed. Having an asymptotic character in a high energy region, they give an infinite number of asymptotic terms. It is proved that these series can be differentiated any number of times with respect to the quasimomentum and preserve their asymptotic character. From the geometrical point of view, we consider the singular set not only as a part of the whole cell $K$, but more precisely, we consider its "trace" on the isoenergetic surface $S_{0}(k)$ in $K$ of the free operator for a fixed energy $k^{2}$. Namely, we study the perturbations of the eigenvalues $p_{j}^{2}(t)$ and their spectral projections, which satisfy the conditions $k^{2}=p_{j}^{2}(t) \approx p_{j+q}^{2}(t)$, for some given $q$ and $k$. It is proved that for a fixed $q$ and sufficiently large $k$ there exists a rich set of $t$ - an essential part of the singular set - for which perturbation series with respect to the model operator converge. Although the main geometrical problems arise because of fixed $k$-one has to prove that perturbation series converge on a rich subset of the surface in $K$ - the consideration of the singular set with a fixed energy turns out to be very useful. It enables us to describe the perturbed isoenergetic surface near the self-intersections of the isoenergetic surface of the free operator (they obviously are formed by the points satisfying the von Laue diffraction conditions). We can observe how these intersections are transformed into quasi-intersections under the perturbation. This approach also turns out to be effective in solving the semicrystal problem (see forthcoming papers). We approximate unstable eigenvalues and eigenfunctions of $H$ by those of a model finite-dimensional operator. This operator roughly takes into account the diffraction inside a crystal and is directly connected with some periodic Schrödinger operator in 
a one-dimensional space. To clarify the results for the singular set we first describe the results for the nonsingular set.

\section{The Perturbation Formulae for the Nonsingular Set.}

It is proved in [1], [2] that there is a rich set of quasimomenta where perturbed and unperturbed eigenvalues and eigenfunctions are close. That is why we call it the nonsingular set. We constructed the nonsingular set $S(k, V, \delta)$ on the isoenergetic surface $S_{0}(k)$ of the free operator., i.e., $S(k, V, \delta) \subset S_{0}(k)$. It is proved that $S(k, V, \delta)$ has an asymptotically full measure on $S_{0}(k)$. To obtain perturbation formulae for $t \in S(k, V, \delta)$ we considered the unperturbed eigenvalue $p_{j}^{2}(t)=k^{2}$. The index $j$ is uniquely defined by the last relation for $t \in S(k, V, \delta)$. Moreover, it is proved that the nearest point $p_{i}^{2}(t), i \neq j$, is on the distance greater than $k^{-1-2 \delta}$ for such t. Then, we consider a circle of radius $(1 / 2) k^{-1-2 \delta}$ centered at the point $z=p_{j}^{2}(t)$. The main result is that a perturbation series for the resolvent converge with respect to some auxiliary operator $\hat{H}$. Note that the series diverge with respect to $H_{0}$, because there are not only the von Laue diffraction conditions, but also another ones, depending on the potential. From the series for the resolvent we easily get series for the perturbed eigenvalues and spectral projection. Replacing $\hat{H}$ with the free operator $H_{0}$ in a long segments of the series, we obtain simplified formulae valid with a high accuracy. To describe the results rigorously we construct the auxiliary operator $\hat{H}(t)$. We also will use it for the case of the singular set. Thus, we construct perturbation series with respect to an auxiliary operator $\hat{H}(t)$. We describe the operator $\hat{H}(t)$ for a trigonometric polynomial (1.2). Let $\Gamma\left(R_{0}\right)$ be the set of $q, q \in Z^{3},|q|<R_{0}$, such that any vector $\vec{p}_{m}(0),|m|<R_{0}$, is a constant multiplier of one and only one vector of $\Gamma\left(R_{0}\right)$. This means that any vector $\vec{p}_{m}(0),|m|<R_{0}$ can be uniquely represented in the form $\vec{p}_{m}(0)=r \vec{p}_{q}(0)$, where $q \in \Gamma\left(R_{0}\right), r \in Z$. It is easy to see that

$$
V=\sum_{q \in \Gamma\left(R_{0}\right)} V_{q}
$$

where $V_{q}$ depends only on $\left(x, \vec{p}_{q}(0)\right)$,

$$
V_{q}=\sum_{r \in Z,|r q|<R_{0}} v_{r q} \exp i r\left(\vec{p}_{q}(0), x\right) .
$$

Let $0<\delta \ll 1 / 5$. In $Z^{3}$ we consider the subsets: 


$$
\begin{gathered}
\Pi\left(k^{1 / 5-8 \delta}\right)=\cup_{q \in \Gamma\left(R_{0}\right)} \Pi_{q}\left(k^{1 / 5-8 \delta}\right), \\
\Pi_{q}\left(k^{1 / 5-8 \delta}\right)=\left\{j:\left|\left(\vec{p}_{j}(0), \vec{p}_{q}(0)\right)\right|<k^{1 / 5-8 \delta}\right\}, \\
T(k, \delta)=\cup_{q, q^{\prime} \in \Gamma\left(R_{0}\right), q \neq q^{\prime}}\left(\Pi_{q}\left(k^{1 / 5-8 \delta}\right) \cap \Pi_{q^{\prime}}\left(k^{3 / 5}\right)\right)=
\end{gathered}
$$

$\left.\left\{j: \exists q, q^{\prime} \in \Gamma\left(R_{0}\right), q \neq q^{\prime}:\left|\left(\vec{p}_{j}(0), \vec{p}_{q}(0)\right)\right|<k^{1 / 5-8 \delta}\right\},\left|\left(\vec{p}_{j}(0), \vec{p}_{q^{\prime}}(0)\right)\right|<k^{3 / 5}\right\}$.

We define the diagonal projection $P_{q}$ and the auxiliary operator $\hat{H}$ as follows:

$$
\left(P_{q}\right)_{j j}=\left\{\begin{array}{l}
1 \text { if } j \in \Pi_{q}\left(k^{1 / 5-8 \delta}\right) \backslash T(k, \delta) \\
0 \text { otherwise }
\end{array}\right.
$$

$$
\hat{H}(t)=H_{0}(t)+\sum_{q \in \Gamma\left(R_{0}\right)} P_{q} V_{q} P_{q}
$$

Let us introduce the notations:

$$
\left.g_{r}(k, t)=\frac{(-1)^{r}}{2 \pi i r} \operatorname{Tr} \oint_{C_{0}}\left(\left(H_{0}\right)-z\right)^{-1} V\right)^{r} d z
$$

$$
G_{r}(k, t)=\frac{(-1)^{r+1}}{2 \pi i} \oint_{C_{0}}\left(\left(H_{0}(t)-z\right)^{-1} V\right)^{r}\left(H_{0}(t)-z\right)^{-1} d z .
$$

$$
\hat{g}_{r}(k, t)=\frac{(-1)^{r}}{2 \pi i r} \operatorname{Tr} \oint_{C_{0}}\left((\hat{H}(t)-z)^{-1} W\right)^{r} d z
$$




$$
\hat{G}_{r}(k, t)=\frac{(-1)^{r+1}}{2 \pi i} \oint_{C_{0}}\left((\hat{H}(t)-z)^{-1} W\right)^{r}(\hat{H}(t)-z)^{-1} d z
$$

$$
T(m)=\frac{\partial^{|m|}}{\partial t_{1}^{m_{1}} \partial t_{2}^{m_{2}} \partial_{3}^{m_{3}}}, \quad m \in R^{3}, \quad|m|=m_{1}+m_{2}+m_{3} .
$$

Here and below $C_{0}$ is the circle of radius $k^{-1-\delta}$ centered at the point $z=k^{2}$. Let $E_{j}$ be the spectral projection of the free operator $H_{0}(t)$ corresponding to eigenvalue $p_{j}^{2}(t):\left(E_{j}\right)_{n m}=\delta_{j n} \delta_{j m}$. The functions $\hat{g}_{r}(k, t)$ and operator-functions $\hat{G}_{r}(k, t)$ are correctly defined in the $\left(k^{-2-2 \delta}\right)$ neighborhood of the nonsingular set $S(k, \delta, V)$. In [1] the following theorem of expansion into the modified perturbation series is proved.

Theorem 2.1. Suppose $t$ belongs to the $\left(k^{-2-2 \delta}\right)$-neighborhood in $K$ of the 1 nonsingular set $S(k, \delta, V), 0<2 \delta<1 / 100$. Then for sufficiently large $k$, $k>k_{0}(V, \delta)$, the interval $\varepsilon(k, \delta) \equiv\left[k^{2}-k^{-1-\delta}, k^{2}+k^{-1-\delta}\right]$ contains a unique eigenvalue of operator $H$. It is given by the absolutely convergent series:

$$
\lambda(t)=p_{j}^{2}(t)+\sum_{r=2}^{\infty} \hat{g}_{r}(k, t)
$$

where the index $j$ is uniquely determined from the relation $p_{j}^{2 l}(t) \in \varepsilon(k, \delta)$. The spectral projection, corresponding to $\lambda(t)$ is determined by the series

$$
E(t)=E_{j}+\sum_{r=1}^{\infty} \hat{G}_{r}(k, t)
$$

which converges in the class $\vec{S}_{1}$.

For functions $\hat{g}_{r}(k, t)$ and the operator-valued functions $\hat{G}_{r}(k, t)$ the following estimates are satisfied:

$$
\begin{gathered}
\left|T(m) \hat{g}_{r}(k, t)\right|<m ! k^{-1-\delta-\gamma_{1} r+2|m|(1+\delta)}, \\
\left\|T(m) \hat{G}_{r}(k, t)\right\|<m ! k^{-\gamma_{1} r+2|m|(1+\delta)} . \\
\gamma_{1}=1 / 15-20 \delta .
\end{gathered}
$$


Furthermore, in [1] it is shown that $\hat{G}_{r}(k, t)=G_{r}(k, t)$, if $r \leq M(k)$ and $\hat{g}_{r}(k, t)=g_{r}(k, t)$, if $r \leq 2 M(k)$, where $M(k)=k^{1-\delta} R_{0}^{-1}$. The next theorem follows from this.

Theorem 2.2. Under the conditions of Theorem 1 the eigenvalue $\lambda(t)$ and its spectral projection $E(t)$ can be represented in the form

$$
\begin{gathered}
\lambda(t)=p_{j}^{2 l}(t)+\sum_{r=2}^{2 M(k)} g_{r}(k, t)+\varphi(k, t), \\
E(t)=E_{j}+\sum_{r=1}^{M(k)} G_{r}(k, t)+\psi(k, t), \\
M(k)=k^{1-\delta} R_{0}^{-1} .
\end{gathered}
$$

For the functions $g_{r}$ and the operator-valued functions $G_{r}$ estimates (2.15) and (2.16) are fulfilled, while for $\varphi(k, t)$ and $\psi(k, t)$, the following ones hold:

$$
\begin{aligned}
& |T(m) \varphi(k, t)|<m ! k^{-\gamma_{1} R_{0}^{-1} k^{1-2 \delta}} k^{2(1+\delta)|m|}, \\
& \|T(m) \psi(k, t)\|_{1}<m ! k^{-\gamma_{1} R_{0}^{-1} k^{1-2 \delta}} k^{2(1+\delta)|m|}
\end{aligned}
$$

Corollary 2.3. For the perturbed eigenvalue and its spectral projection the following estimates are satisfied:

$$
|T(m)|\left(\lambda(t)-p_{j}^{2}(t)\right) \mid<c m ! k^{-1-\delta-2 \gamma_{1}+2|m|(1+\delta)}
$$

$$
\left\|T(m)\left(E(t)-E_{j}\right)\right\|<c m ! k^{-\gamma_{1}+2|m|(1+\delta)} .
$$

Note that for $r, m$ small enough estimates (2.15), (2.16), (2.21), (2.22) can be improved so that there will be only negative powers of $k$ on the right sides. 


\section{The Perturbation Formulae Near the Planes of Diffraction.}

In the case of the polyharmonic operator with a periodic potential we constructed the perturbation series with respect to the free operator on the nonsingular set [7] and with respect to a model operator on the singular set [8]. This model operator roughly took into account the diffraction inside the crystal. In the case of the Schrödinger operator the modification of the perturbation series $\left(H_{0} \rightarrow \hat{H}\right)$ is needed even for the nonsingular set [1]. The series diverge with respect to $H_{0}$, because there are not only the von Laue diffraction conditions, but also another ones, depending on the potential. Fortunately, it turns out that such modified series converge even on the essential part of the singular set, i.e., it is not necessary to reconstruct additionally these series. The perturbation series for the eigenvalue and its spectral projection converge on the essential part of the singular set, when we take the operator $\hat{H}(t)$ as the initial one. Note that the operator $H(t)$ has a block structure. The diagonal part of $\hat{H}$ coincides with the corresponding part of the free operator: $\left(I-\sum_{q \in \Gamma\left(R_{0}\right)} P_{q}\right) \hat{H}(t)=\left(I-\sum_{q \in \Gamma\left(R_{0}\right)} P_{q}\right) H_{0}(t)$. The blocks of $\hat{H}(t)$ are determined by the orthogonal projections $P_{q}, P_{q} \hat{H}(t)=\hat{H}(t) P_{q}=P_{q} \hat{H}(t) P_{q}$. Each block is a "piece" of the matrix of the Schrödinger operator with the potential $V_{q}$, i.e., $P_{q} \hat{H}(t) P_{q}=P_{q}\left(H_{0}(t)+V_{q}\right) P_{q}$. Thus, each block is simply connected with the matrix of a periodic Schrödinger operator in the one-dimensional space, because $V_{q}$ depends only on $\left(x, \vec{p}_{q}(0)\right)$ In the case of the nonsingular set the perturbed eigenvalue $\lambda(t)$ is asymptotically close $(k \rightarrow \infty)$ to an eigenvalue $p_{j}^{2 l}(t)$ of the diagonal part of $\hat{H}(t)$; i.e, $\lambda(t)$ is close to the eigenvalue $p_{j}^{2 l}(t)$ of the free operator $H_{0}(t)$. Accordingly, the spectral projector of $H(t)$, corresponding to $\lambda(t)$, is close to that of $H_{0}(t)$, corresponding to $p_{j}^{2 l}(t)$. We prove that in the case of the singular set (more precisely of its essential part) eigenvalues and spectral projection of $H(t)$ are close to those of the block part of $\hat{H}(t)$. We construct the perturbation series for an eigenvalue and its spectral projection on the essential part of the singular set, taking $\hat{H}(t)$ as the initial operator. We obtain this result by constructing the converging perturbation series for the resolvent and integrating it over a small contour. Thus, the blocks $P_{q} \hat{H} P_{q}=P_{q}\left(H_{0}(t)+V_{q}\right) P_{q}$ describe roughly the refraction inside the crystal for $t$ of the essential part of the singular set. We call this essential part of the singular set the simple part of the singular set, because on the relatively small rest of the singular set the picture of diffraction is even more complicated. 
The proof of the formulae contains the analytical and geometrical parts. In the analytical part we formulate the conditions of the convergence of the series in the form of the inequalities for quasimomentum $t$ (see (3.7)(3.12). Note that these conditions are similar to those for the nonsingular set up to the replacement of an eigenvalue $p_{j}^{2}(t)$ of the diagonal part of $\hat{H}(t)$ by an eigenvalue of the block part. In the geometrical part we prove that the conditions are satisfied on the essential part of the singular set. Note that the analytical part is similar to that for the nonsingular set up to the mentioned above replacement. The geometrical part is much more technical. The singular set is only a small part of the isoenergetic surface of the free operator. Therefore, to prove that the conditions of convergence can be satisfied on it, one has to make more subtle considerations than in the case of the nonsingular set.

Thus, we consider the spherical layer on the sphere $|x|=k$ :

$$
S_{q}(k,-\delta)=\left\{x:|x|=k,\left.|| x\right|^{2}-\left|x+\vec{p}_{q}(0)\right|^{2} \mid<k^{\delta}\right\},
$$

Let $\mathcal{K}$ be the mapping

$$
\mathcal{K}: R^{3} \rightarrow K, \quad \mathcal{K} \vec{p}_{j}(t)=t .
$$

It is easy to see that the isoenergetic surface of the free operator $S_{0}(k)$ is given by the formula $S_{0}(k)=\mathcal{K} S_{k}$, where $S_{k}$ is the sphere $|x|=k$. It is clear that the translation $\mu_{q}(k, \delta) \equiv \mathcal{K} S_{q}(k,-\delta)$ of the set $S_{q}(k,-\delta)$ to the elementary cell of the dual lattice $K$ belongs to the isoenergetic surface $S_{0}(k)$ of the free operator $H_{0}$, but does not belong to the nonsingular set, described in [1], where perturbed and unperturbed eigenvalues are proved to be close. Next, we denote the eigenvalues of $\hat{H}$ (see (2.6)) as $p_{j}^{2}(t)+\Delta \hat{\Lambda}_{j j}(t)$, $\Delta \hat{\Lambda}_{j j}(t)$ depending continuously on $t$ for every $j$. It is clear that $\Delta \hat{\Lambda}_{j j}=0$ when $j \in \Pi_{q}\left(k^{1 / 5}\right) \backslash T(k, \delta)$. Let us consider also the part $\hat{\mu}_{q}(k, \delta)$ of the isoenergetic surface of $\hat{H}$ situated in the $\left(c k^{-1}\right)$-neighborhood of $\mu_{q}(k, \delta)$. It is described by the formula:

$$
\hat{\mu}_{q}(k, \delta)=\left\{t: \exists j, j \in Z^{3}, p_{j}^{2}(t)+\Delta \hat{\Lambda}_{j j}(t)=k^{2},\left|p_{j}^{2}(t)-p_{j+q}^{2}(t)\right|<k^{\delta}\right\} .
$$

In fact, $\hat{\mu}_{q}(k, \delta)$ is very close to the corresponding part of the isoenergetic surface of the operator $H_{q}=-\Delta+V_{q}$, because $p_{j}^{2}(t)+\Delta \hat{\Lambda}_{j j}(t)$ coincides 
with an eigenvalue $p_{j}^{2}(t)+\Delta \Lambda_{j j}(t)$, of $H_{q}(t)$ up to a value of order $k^{-c k^{1 / 5-8 \delta}}$ (see [1]). All following assertions are also valid for $\hat{\mu}_{q}(k, \delta)$ being the corresponding part of the isoenergetic surface of $H_{q}$.

Note, that $\hat{\mu}_{q}(k, \delta)=\mathcal{K} \hat{S}_{q}(k,-\delta)$, where

$$
\hat{S}_{q}(k,-\delta)=\left\{x:|x|^{2}+\varphi_{0}(x)=k^{2},\left.|| x\right|^{2}-\left|x+\vec{p}_{q}(0)\right|^{2} \mid<k^{\delta}\right\},
$$

$\varphi_{0}(x)$ being uniquely determined from formulae

$$
\varphi_{0}(x)=\Delta \hat{\Lambda}_{j j}(t), \quad x=\vec{p}_{j}(t) .
$$

It is not hard to show that $\hat{S}_{q}(k,-\delta)$ is the union of curve cylinders, because $\Delta \hat{\Lambda}_{j j}$, in fact, depends only on the projection of $\vec{p}_{j}(t)$ on $\vec{p}_{q}(t)$. Note that $\Delta \Lambda_{j j} \neq 0$ in (3.3), because $j \in \Pi_{q}\left(k^{1 / 5}\right)$. We construct perturbation series with respect to $\hat{H}(t)$. In the case of the nonsingular set the corresponding pieces of the isoenergetic surfaces of $H_{0}(t)$ and $\hat{H}(t)$ coincide. Clearly, describing the nonsingular set on the isoenergetic surface of one of them we, of course, describe for the other too. In the case of the nonsingular set these surfaces are not the same, i.e., $\mu_{q}(k, \delta)$ does not coincides with $\hat{\mu}_{q}(k, \delta)$. Therefore, the question is : on which isoenergetic surface the simple part of the singular set should be described? Since we construct perturbation series with respect to $\hat{H}(t)$, it is natural to describe this set on $\hat{\mu}_{q}(k, \delta)$. We formulate the corresponding geometric lemma here. It will be proved in Section 6 . We also describe the simple part of the nonsingular set on $\mu_{q}(k, \delta)$ there. However, the first description is more convenient for applications. Thus, we shall show that there exists a subset $\hat{\chi}_{q}^{0}(k, V, \delta)$ of an asymptotically full measure on $\hat{\mu}_{q}(k, \delta)$, such that for $t \in \hat{\chi}_{q}^{0}(k, V, \delta)$ the perturbation series with respect to $\hat{H}(t)$ converge. For such a $t$ the operator $\hat{H}(t)$ has an eigenvalue which can be represented in the form $p_{j}^{2}(t)+\Delta \hat{\Lambda}(t)_{j j}$, $\mathrm{j}$ being uniquely determined from the relation $p_{j}^{2}(t)+\Delta \hat{\Lambda}(t)_{j j}=k^{2}$. The series for $H(t)$ converge with respect to $\hat{H}(t)$. Now we give the formulation of the main geometric lemma.

Lemma 3.1. Let $0<\delta<1 / 300$. Then for sufficiently large $k, k>k_{0}(V, \delta)$, there exists a set $\hat{\chi}_{q}^{0}(k, V, \delta) \subset \hat{\mu}_{q}(k, \delta)$, such that for any $t$ of this set there is a unique $j \in Z^{3}$ such that $p_{j}^{2}(t)+\Delta \hat{\Lambda}(t)_{j j}=k^{2}$,

$$
\left|p_{j}^{2}(t)-p_{j+q}^{2}(t)\right|<k^{\delta}
$$


and the following conditions hold:

$1^{\prime}$

$$
\min _{r \in \Omega_{1}}\left|p_{j}^{2}(t)+\Delta \hat{\Lambda}(t)_{j j}-p_{j+m}^{2}(t)\right|>k^{1-1.0 \delta}
$$

$$
\Omega_{1}=\left\{m: m \in Z^{3},|m|<k^{\delta} ; m \neq 0 ; m \neq n_{0} q, n_{0} \in Z\right\} .
$$

$2^{\prime}$

$$
\left|p_{m+j+i}^{2}(t)-p_{j}^{2}(t)-\Delta \hat{\Lambda}(t)_{j j}\right|>k^{-12 \delta}\left|p_{m+j}^{2}(t)-p_{j}^{2}(t)-\Delta \hat{\Lambda}(t)_{j j}\right|^{-1} .
$$

for all $m:\left|p_{m+j}^{2}(t)-p_{j}^{2}(t)-\Delta \hat{\Lambda}(t)_{j j}\right|<k^{-20 \delta}, m \neq n_{0} q, n_{0} \in Z$, and $|i|<k^{\delta}, i \neq 0$.

$3^{\prime}$

$$
\min _{m \in Z^{3}, m \neq n_{0} q, n_{0} \in Z}\left|p_{m+j}^{2}(t)-p_{j}^{2}(t)-\Delta \hat{\Lambda}(t)_{j j}\right|>2 k^{-1-\delta}
$$

$4^{\prime}$. For any $q^{\prime}$ in $\Gamma\left(R_{0}\right)$ and $m \in \Pi_{q^{\prime}}\left(k^{1 / 5}\right)$ the eigenvalue $p_{m}^{2}(t)+$ $\Delta \hat{\Lambda}(t)_{m m}$ of the operator $\hat{H}_{q}(t)$ lies sufficiently far from the point $p_{j}^{2}(t)+$ $\Delta \hat{\Lambda}(t)_{j j}$, namely,

$$
\left|p_{m}^{2}(t)+\Delta \hat{\Lambda}(t)_{m m}-p_{j}^{2}(t)-\Delta \hat{\Lambda}(t)_{j j}\right|>\left|\left(\vec{p}_{m}(0), \vec{p}_{q^{\prime}}(0)\right)\right|^{-1+\delta} .
$$

$5^{\prime}$ If $m \in T(k, \delta)$, then

$$
\left|p_{m}^{2}(t)-p_{j}^{2}(t)-\Delta \hat{\Lambda}(t)_{j j}\right|>k^{1 / 5-9 \delta} .
$$

These properties hold in a small neighbourhood of $\hat{\chi}_{q}^{0}(k, V, \delta)$ : if $t$ is in the $\left(k^{-2-2 \delta}\right)$-neighbourhood of $\left.\hat{\chi}_{q}^{0}(k, V, \delta)\right)$, then there is a unique $j \in Z^{3}$ such that $\left|p_{j}^{2}(t)+\Delta \hat{\Lambda}(t)_{j j}-k^{2}\right|<k^{-1-2 \delta}$, inequality (3.6) and conditions $1^{\prime}-5^{\prime}$ are satisfied. 
The set $\hat{\chi}_{q}^{0}(k, V, \delta)$ has an asymptotically full measure on $\hat{\mu}_{q}(k, \delta)$; moreover the inequality

$$
\frac{s\left(\hat{\mu}_{q}(k, \delta) \backslash \hat{\chi}_{q}^{0}(k, V, \delta)\right)}{s\left(\hat{\mu}_{q}(k, \delta)\right)}<k^{-3 \delta}
$$

is valid.

Conditions $1^{\prime}-5^{\prime}$ coincide with conditions $1^{0}-5^{0}$ of the convergence of the series in [1] up to replacement of $p_{j}^{2}(t)$ by $p_{j}^{2}(t)+\Delta \hat{\Lambda}(t)_{j j}$. Note, that inequalities (3.10) and (3.11) yield that the eigenvalue $p_{j}^{2}(t)+\Delta \hat{\Lambda}(t)_{j j}$ of operator $\hat{H}(t)$ is simple. We denote its spectral projection by $\hat{E}_{j}$. Let

$$
\hat{g}_{r}^{\prime}(k, t)=\frac{(-1)^{r}}{2 \pi i r} \operatorname{Tr} \oint_{C_{1}} \hat{A}(t)^{r} d z
$$

$$
\hat{G}_{r}^{\prime}(k, t)=\frac{(-1)^{r+1}}{2 \pi i} \oint_{C_{1}}(\hat{H}(t)-z)^{-1 / 2} \hat{A}^{r}(t)(\hat{H}(t)-z)^{-1 / 2} d z,
$$

where $C_{1}$ is the circle of radius $k^{-1-\delta}$ centered at the point $\lambda=p_{j}^{2}(t)+$ $\Delta \hat{\Lambda}(t)_{j j}$,

$$
\hat{A}(z, t, W)=(\hat{H}(t)-z)^{-1 / 2} W(\hat{H}(t)-z)^{-1 / 2},
$$

$W$ being given by formula (2.7).

Theorem 3.2. Suppose $0<\delta<1 / 300, t$ is in the $\left(k^{-2-2 \delta}\right)$-neighborhood of set $\hat{\chi}_{q}^{0}(k, V, \delta)$. Then for sufficiently large $k, k>k_{0}(V, \delta)$, there exists a unique eigenvalue of the operator $H(t)$ in the $\left(k^{-1-2 \delta}\right)$-neighborhood of the point $\lambda=p_{j}^{2}(t)+\Delta \hat{\Lambda}(t)_{j j}$. It is given by the absolutely converging series:

$$
\hat{\lambda}(t)=p_{j}^{2}(t)+\Delta \hat{\Lambda}(t)_{j j}+\sum_{r=2}^{\infty} \hat{g}_{r}^{\prime}(k, t)
$$

The spectral projection corresponding to $\hat{\lambda}(t)$ is determined by the series:

$$
\hat{E}(t)=\hat{E}_{j}+\sum_{r=1}^{\infty} \hat{G}_{r}^{\prime}(k, t)
$$


which converges in the class $\vec{S}_{1}$. For the functions $\hat{g}_{r}^{\prime}(k, t)$ and the operatorvalued functions $\hat{G}_{r}^{\prime}(k, t)$ the following estimates hold:

$$
\begin{gathered}
\left|\hat{g}_{2}^{\prime}(k, t)\right|<k^{-8 / 5+7 \delta}, \\
\left\|\hat{G}_{1}^{\prime}(k, t)\right\|_{1}<k^{-1+4 \delta}, \\
\left\|\hat{G}_{2}^{\prime}(k, t)\right\|_{1}<k^{-7 / 5+6 \delta}, \\
\left|\hat{g}_{r}(k, t)\right|<k^{-1-\delta-\gamma_{1} r}, \\
\left\|\hat{G}_{r}(k, t)\right\|_{1}<k^{-\gamma_{1} r}
\end{gathered}
$$

\section{Corollary 3.3.}

$$
\begin{gathered}
\left|\hat{\lambda}(t)-p_{j}^{2}(t)-\Delta \hat{\Lambda}(t)_{j j}\right|<c k^{-1-2 \gamma_{1}}, \\
\left\|\hat{E}(t)-E_{j}\right\|<c k^{-3 \gamma_{1}}, c \neq c(k) .
\end{gathered}
$$

Theorem 3.4. Under the conditions of Theorem 3 the functions $\hat{g}_{r}^{\prime}(k, t)$ and the operator-valued functions $\hat{G}_{r}^{\prime}(k, t)$ depend analytically on $t$ in the complex $\left(k^{-2-2 \delta}\right)$-neighborhood of each simply connected component of $\hat{\chi}_{q}^{0}(k, V, \delta)$. They satisfy the estimates:

$$
\begin{gathered}
\left|T(m) \hat{g}_{2}^{\prime}(k, t)\right|<m ! k^{-8 / 5+7 \delta+2(1+\delta)|m|}, \\
\left\|T(m) \hat{G}_{1}^{\prime}(k, t)\right\|<m ! k^{-1+4 \delta+2(1+\delta)|m|}, \\
\left\|T(m) \hat{G}_{2}^{\prime}(k, t)\right\|<m ! k^{-7 / 5+6 \delta+2(1+\delta)|m|}, \\
\left|T(m) \hat{g}_{r}^{\prime}(k, t)\right|<m ! k^{-1-\delta-\gamma_{1} r+2(1+\delta)|m|}, \\
\left\|T(m) \hat{G}_{r}^{\prime}(k, t)\right\|<m ! k^{-\gamma_{1} r+2(1+\delta)|m|},
\end{gathered}
$$


Corollary 3.5. The function $\hat{\lambda}(t)$ and the operator-valued function $\hat{E}(t)$ analytically depend on $t$ in the complex $\left(k^{-2-2 \delta}\right)$-neighborhood of each simply connected component of the set $\hat{\chi}_{q}^{0}(k, V, \delta)$. They satisfy the following estimates:

$$
\begin{gathered}
\left|T(m)\left(\hat{\lambda}(t)-p_{j}^{2}(t)-\Delta \hat{\Lambda}(t)_{j j}\right)\right| \leq c m ! k^{-1-2 \gamma_{1}+2(1+\delta)|m|} \\
\left\|T(m)\left(\hat{E}(t)-\hat{E}_{j}\right)\right\|_{1} \leq c m ! k^{-3 \gamma_{1}+2(1+\delta)|m|}
\end{gathered}
$$

Note that for $r, m$ small enough, estimates (3.25) - (3.31) can be improved so that there will be only negative powers of $k$ on the right sides. Theorems 3 and 4 enable us to conclude, that the points, satisfying the equation $\hat{\lambda}(t)=$ $k^{2}$, are situated near the isoenergetic surface of the operator $\hat{H}$.

The similar results hold for $\mu_{q}(k, \delta)$, which belongs to the isoenergetic surface of the free operator.

Lemma 3.6. Let $0<\delta<1 / 300$. Then for sufficiently large $k, k>k_{0}(V, \delta)$, there exists a set $\hat{\chi}_{q}^{\prime}(k, V, \delta) \subset \mu_{q}(k, \delta)$, such that for any $t$ in its $\left(k^{-2-2 \delta}\right)$ neighborhood there exists a unique $j \in Z^{3}$ such that

$$
\left|p_{j}^{2}(t)-k^{2}\right|<k^{-1-\delta} \quad\left(p_{j}^{2}(t)=k^{2} \text { for } t \text { of } \hat{\chi}_{q}^{\prime}(k, V, \delta)\right),
$$

inequality (3.6) and the conditions $1^{\prime}-5^{\prime}$ are satisfied: The set $\hat{\chi}_{q}^{\prime}(k, V, \delta)$ has an asymptotically full measure on $\mu_{q}(k, \delta)$; moreover the inequality

$$
\frac{s\left(\mu_{q}(k, \delta) \backslash \hat{\chi}_{q}^{\prime}(k, V, \delta)\right)}{s\left(\mu_{q}(k, \delta)\right)}<k^{-3 \delta}
$$

holds.

The proof of this lemma is similar to that of Lemma 1. Naturally, for $\hat{\chi}_{q}^{\prime}(k, V, \delta)$ the theorems similar to Theorems 3 and 4 hold:

Theorem 3.7. Suppose $0<\delta<1 / 300$ and $t$ is in the $\left(k^{-2-2 \delta}\right)$ neighborhood of the set $\hat{\chi}_{q}^{\prime}(k, V, \delta)$ Then, for sufficiently large $k, k>k_{0}(V)$ there exists a unique eigenvalue $\hat{\lambda}(t)$ of operator $H(t)$ in the $\left(k^{-2-2 \delta}\right)$ neighborhood of the point $p_{j}^{2}(t)$. It is given by the absolutely converging series (3.16), $j$ being uniquely determined by the relation (3.32). The corresponding spectral projection is given by formula (3.17). Estimates (3.18)(3.22) hold. 
Corollary 3.8. If $t$ is in the $\left(k^{-2-2 \delta}\right)$-neighborhood of $\hat{\chi}_{q}^{\prime}(k, V, \delta)$, then estimates (3.23)-(3.24) hold.

Theorem 3.9. Under the conditions of Theorem 5 the functions $\hat{g}_{r}^{\prime}(k, t)$ and the operator-valued functions $\hat{G}_{r}^{\prime}(k, t)$ depend analytically on $t$ in the complex $\left(k^{-2-2 \delta}\right)$-neighborhood of each simply connected component of the set $\hat{\chi}_{q}^{\prime}(k, V, \delta)$. They satisfy estimates (3.25) - (3.29).

Corollary 3.10. The function $\hat{\lambda}(t)$ and the operator-valued function $\hat{E}(t)$ analytically depend on $t$ in the complex $\left(k^{-2-2 \delta}\right)$-neighborhood of each simply connected component of the set $\hat{\chi}_{q}^{\prime}(k, V, \delta)$. They satisfy the estimates (3.30), (3.31).

\section{The behavior of the isoenergetic surface in a vicinity of the singular set.}

Since the eigenvalues and the spectral projections of operator $H$ are close to those of $\hat{H}$ on the essential part of the singular set, the isoenergetic surface of $H$ is close to that of $\hat{H}$. We denote by $\hat{\mu}_{q}(k, t)_{j}$ the part of $\hat{\mu}_{q}(k, \delta)$, corresponding to some given $j$; i.e., point $t$ belongs to $\hat{\mu}_{q}(k, \delta)_{j}$ if and only if

$$
p_{j}^{2}(t)+\Delta \hat{\Lambda}(t)_{j j}=k^{2} .
$$

Let $\vec{p}_{j \perp}(t)$ be the component of the vector $\vec{p}_{j}(t)$ orthogonal to $\vec{p}_{q}(0)$. Equation (4.1) is resolvable with respect to $\left|\vec{p}_{j \perp}(t)\right|$, since $\hat{\Delta} \Lambda(t)_{j j}$ depends only on $\left(\vec{p}_{j}(t), \vec{p}_{q}(0)\right)$ :

$$
\left|\vec{p}_{j \perp}\right|=f_{0}\left(\left(\vec{p}_{j}(t), \vec{p}_{q}(0)\right)\right)
$$

$$
f_{0}\left(\left(\vec{p}_{j}(t), \vec{p}_{q}(0)\right)\right)=\left(k^{2}-\Delta \hat{\Lambda}(t)_{j j}-\left(\vec{p}_{j}(t), \vec{p}_{q}(0)\right)^{2} p_{q}(0)^{-2}\right)^{1 / 2}
$$

Thus, point of $\hat{S}_{q}(k,-\delta)$ can be represented by formula for a curve cylinder:

$$
x_{\perp}=f_{0}\left(x_{\|}\right), \quad x_{\|}=\left(x, \vec{p}_{q}(0)\right) p_{q}^{-1}(0) .
$$

To obtain $\hat{\mu}_{q}(k, \delta)$ one has to break this curve cylinder into pieces by the dual lattice and to translate all pieces into elementary cell of the dual lattice. According to Theorem 3 there exists a subset $\hat{\chi}_{q}^{0}(k, V, \delta)$ of an asymptotically 
full measure on $\hat{\mu}_{q}(k, \delta)$ on which the perturbation series converge. We denote by $\hat{\chi}_{q}^{0}(k, V, \delta)_{j}$ the intersection of $\hat{\chi}_{q}^{0}(k, V, \delta)$ with $\hat{\mu}_{q}(k, \delta)_{j}$. In Lemma 6 we will prove that the $\left(k^{-2-2 \delta}\right)$-neighborhoods of pieces $\hat{\chi}_{q}^{0}(k, V, \delta)_{j}$ do not intersect with each other for different $j$. This means that there are no $\hat{\chi}_{q}^{0}(k, V, \delta)_{i}, i \neq j$ in the $\left(k^{-2-2 \delta}\right)$-neighborhood of $\hat{\chi}_{q}^{0}(k, V, \delta)_{j}$.

Let $\hat{e}(t)$ be the unit vector orthogonal to $\hat{\mu}_{q}(k, \delta)$ at point $t$.

Theorem 4.1. For $0<\delta<1 / 300$ and sufficiently large $k, k>k_{0}(V, \delta)$ there exists a unique piece $\hat{S}_{H}^{\prime}(k)$ of the isoenergetic surface of $H$ in the $\left(k^{-2-2 \delta}\right)$-neighborhood of each simply connected component of $\hat{\chi}_{q}^{0}(k, V, \delta)$. In fact, it is in the smaller $\left(k^{-2-\gamma_{2}}\right)$-neighborhood of $\hat{\chi}_{q}^{0}(k, V, \delta)$ and can be described by the equation

$$
\hat{\lambda}(t)=k^{2},
$$

where the function $\hat{\lambda}(t)$ is determined by the converging series (3.16). The corresponding spectral projection is given by formula (3.17). The terms of these series are satisfied to estimates (3.18) -(3.22), (3.28) and (3.29).

The normal e $(t)$, to $S_{H}^{\prime}(k)$ at a point $t$ satisfies the asymptotic:

$$
e(t)=\hat{e}(t)+O\left(k^{-2 \gamma_{1}}\right) .
$$

Thus, $\hat{S}_{H}^{\prime}$ is close to the corresponding part of the isoenergetic surface of the Schrödinger operator $H_{q}=H_{0}+V_{q}$, where $V_{q}$ depends only on $\left(x, \vec{p}_{q}(0)\right)$ (see (2.1)).

The short version of results is already announced in [9]. Here we represent the proofs.

\section{Proof of the Perturbation Formulae on the Singular Set.}

The formal construction of the perturbation series on the singular set is quite similar to that on the nonsingular set. The proof of convergence is based on the conditions $1^{\prime}-5^{\prime}$, which are analogous to the conditions $1^{0}-5^{0}$ on the nonsingular set, and so it is similar to the proof of Theorem 1 in [1]. In this section we prove Lemma 4, which, in fact, asserts the convergence of the perturbation series for the resolvent of the operator $H(t)$ with respect to the model operator $\hat{H}(t)$. The validity of estimates (3.18)-(3.20) is verified in Lemma 5. Basing on these two lemmas we prove Theorems 3 and 4.

Lemma 5.1. If $t$ is in the $\left(k^{-2-2 \delta}\right)$-neighborhood of the set $\hat{\chi}_{q}^{0}(k, V, \delta)$, then the operator $\hat{H}(t)$ has a unique eigenvalue inside the circle $C_{1}$. For any 
$z \in C_{1}$ the following estimate holds:

$$
\left\|(\hat{H}(t)-z)^{-1 / 2}\right\|<k^{1 / 2+2 \delta} .
$$

Proof. We first prove estimate (5.1). According to Lemma 1 for any $t$ of $\hat{\chi}_{q}^{0}(\overline{k, V, \delta})$ there exists a unique $j \in Z^{3}$ such that $p_{j}^{2}(t)+\Delta \hat{\Lambda}_{j j}(t)=k^{2}$. By the definition of the circle $C_{1}$ the point $k^{2}=p_{j}^{2}(t)+\Delta \hat{\Lambda}_{j j}(t)$ is at its center and

$$
\left|p_{j}^{2}(t)+\Delta \hat{\Lambda}_{j j}(t)-z\right|=k^{-1-\delta}, \quad z \in C_{1} .
$$

Let $P=\sum_{q \in \Gamma\left(R_{0}\right)} P_{q}$. Suppose $i: P_{i i}=0$. Than, using (3.10) and (5.2), we get

$$
\left|p_{i}^{2}(t)-z\right|>k^{-1-\delta}
$$

Since $\hat{H}(t)$ acts as $H_{0}(t)$ in $(I-P) l_{2}^{3}$ and inequality (5.3) holds, we easily obtain

$$
\left\|(I-P)(\hat{H}(t)-z)^{-1 / 2}\right\|<k^{1 / 2+\delta / 2} .
$$

Suppose $i: P_{i i}=1, i \neq j$. It is clear that

$$
\left|p_{i}^{2}(t)+\Delta \hat{\Lambda}_{i i}(t)-z\right|>
$$

$$
\left|p_{i}^{2}(t)+\Delta \hat{\Lambda}_{i i}(t)-p_{j}^{2}(t)-\Delta \hat{\Lambda}_{j j}(t)\right|-\left|p_{j}^{2}(t)+\Delta \hat{\Lambda}_{j j}(t)-z\right| .
$$

Using relations (3.11) and noting that $\left|\left(\vec{p}_{i}(0), \vec{p}_{q^{\prime}}(0)\right)\right|<k^{1 / 5-8 \delta}$ when $q^{\prime} \in$ $\Pi_{q^{\prime}}\left(k^{1 / 5-8 \delta}\right)$ (see (2.3), we get

$$
\left|p_{i}^{2}(t)+\Delta \hat{\Lambda}_{i i}(t)-p_{j}^{2}(t)-\Delta \hat{\Lambda}_{j j}(t)\right|>2 k^{-1 / 5-6 \delta} .
$$

Taking into account (5.2), we easily obtain:

$$
\left|p_{i}^{2}(t)+\Delta \hat{\Lambda}_{i i}(t)-z\right|>k^{-1 / 5-6 \delta} \quad i \neq j .
$$

Note that $p_{i}^{2}(t)+\Delta \hat{\Lambda}_{i i}(t)$ are the eigenvalues of $P \hat{H} P$. Therefore,

$$
\|\left(P(\hat{H}(t)-z)^{-1 / 2} \|<k^{1 / 10+3 \delta} .\right.
$$

Inequalities (5.4), (5.8) together give estimate (5.1).

Now we prove that $\hat{H}$ has a unique eigenvalue inside $C_{1}$. It follows from relations (3.10) and (5.7) that the points $p_{i}^{2}(t), i: P_{i i}=0$ and $p_{i}^{2}(t)+\Delta \hat{\Lambda}_{i i}(t)$, $i: P_{i i}=1, i \neq j$ lie outside the circle $C_{1}$. Since they are all eigenvalues of operator $\hat{H}(t)$, this means that there are an unique eigenvalue of operator $\hat{H}(t)$ inside this circle. It is equal to $p_{j}^{2}(t)+\Delta \Lambda_{j j}(t)$. The lemma is proved. 
Lemma 5.2. Suppose $t$ is in the $\left(k^{-2-2 \delta}\right)$-neighbourhood of the set $\hat{\chi}_{q}^{0}(k, V, \delta), z \in C_{1}$. Then for sufficiently large $k, k>k_{0}(V, \delta)$, the following estimates hold:

$$
\begin{gathered}
\|\hat{A}(z, t)\|<k^{8 \delta}, \\
\left\|\hat{A}^{3}(z, t)\right\|<k^{-1 / 5+31 \delta .}
\end{gathered}
$$

Proof. We consider the operator $A_{1}$ :

$$
A_{1}=A_{0}+A_{1}^{(1)}+A_{1}^{(2)}+A_{3}^{(3)},
$$

where

$$
\begin{gathered}
A_{1}^{(i)}=\left(H_{0}(t)+\Delta \hat{\Lambda}-z\right)^{-1 / 2} B_{1}^{(i)}\left(H_{0}(t)+\Delta \hat{\Lambda}-z\right)^{-1 / 2}, \\
B_{1}^{(1)}=P_{0} \mathcal{E}_{q}\left(I-P_{0}\right) \\
B_{1}^{(2)}=B_{1}^{(1) *}=\left(I-P_{0}\right) \mathcal{E}_{q} P_{0} \\
B_{1}^{(3)}=P_{0} \mathcal{E}_{q} P_{0}
\end{gathered}
$$

$$
\begin{gathered}
A_{0}=\left(H_{0}-z\right)^{-1 / 2} B_{0}\left(H_{0}-z\right)^{-1 / 2}, \\
B_{0}=(I-P) V(I-P),
\end{gathered}
$$

$P_{0}$ being the diagonal one-dimensional projection: $\left(P_{0}\right)_{j j}=1$. The formal description of $\mathcal{E}_{q}$ is given in [1]. Its main properties are the following:

$$
\begin{gathered}
\mathcal{E}_{q}=\mathcal{E}_{q}^{*}, \quad\left\|\mathcal{E}_{q}\right\|<\|V\|, \\
\left(\mathcal{E}_{q}\right)_{i j}=0, \quad \text { if } i-j \neq l_{0} q+l q^{\prime},
\end{gathered}
$$

where $q^{\prime} \neq q, q^{\prime} \in \Gamma\left(R_{0}\right), \quad l_{0}, l \in R, \quad\left|l_{0}\right|<k^{1 / 5-8 \delta}, 1 \leq|l|<R_{0}$, 


$$
P_{k^{2}}\left(k^{1 / 5-8 \delta}\right) \mathcal{E}_{q} P_{k^{2}}\left(k^{1 / 5-8 \delta}\right)=0 .
$$

Here and below $P_{k^{2}}\left(k^{1 / 5-8 \delta}\right)$ is the diagonal projection

$$
P_{k^{2}}\left(k^{1 / 5-8 \delta}\right)_{i i}= \begin{cases}1, & \text { if } 2\left|p_{i}^{2}(t)-k^{2}\right|<k^{1 / 5-8 \delta}, \\ 0, & \text { otherwise. }\end{cases}
$$

To prove estimates (5.9), (5.10) we verify the following relations:

1)

$$
\left\|A_{1}\right\|<k^{7 \delta}
$$

2)

$$
\left\|\hat{A}-A_{1}\right\|<5 k^{-1 / 5+8 \delta}
$$

3)

$$
\left\|A_{1}^{3}\right\|<8 k^{-1 / 5+30 \delta}
$$

First, we check that

$$
A_{1}^{(3)}=B_{1}^{(3)}=0 .
$$

Indeed, from relation (5.20), taking into account that $P_{0} P_{k^{2}}\left(k^{1 / 5-8 \delta}\right)=P_{0}$, we obtain $B_{1}^{(3)}=0$ and, therefore $A_{1}^{(3)}=0$.

1) We prove inequality (5.22). Let us estimate $\left\|A_{0}\right\|$. It is clear that

$$
\left(A_{0}\right)_{m i}=v_{m-i}\left(p_{m}^{2}(t)-z\right)^{-1 / 2}\left(p_{i}^{2}(t)-z\right)^{-1 / 2} .
$$

We prove that

$$
\begin{gathered}
\left|\left(p_{m}^{2}(t)-z\right)\left(p_{i}^{2}(t)-z\right)\right|>k^{-4 \delta} \\
i, m \in Z^{3}, i \neq m,|i-m|<k^{\delta}, P_{m m}=P_{i i}=0 .
\end{gathered}
$$

for all $z \in C_{1}$. Inequality (5.27) is symmetric with respect to $m, i$, so we can assume that $\left|p_{m}^{2}(t)-z\right| \leq\left|p_{i}^{2}(t)-z\right|$. If $\left|p_{m}^{2}(t)-z\right|>k^{-2 \delta}$, then inequality (5.27) is obvious. Suppose

$$
\left|p_{m}^{2}(t)-z\right| \leq k^{-2 \delta}
$$

Let $m \neq j+n_{0} q, n_{0} \in Z$. Using inequalities (3.7)-(3.10) and considering as in the proof of Corollary of the Geometric Lemma in [7] we verify that 
relation (5.27) holds. Next, let $m=j+n_{0} q, n_{0} \in Z$. Suppose, $\left|n_{0}\right|>$ $k^{1 / 5-9 \delta}$. Then $\left|p_{m}^{2}(t)-p_{j}^{2}(t)\right|>c k^{1 / 5-9 \delta}$, because $\left|\left(\vec{p}_{j}(t), \vec{p}_{q}(0)\right)\right|<k^{1 / 5-10 \delta}$. Considering relation (5.2), we obtain that

$$
\left|p_{m}^{2}(t)-z\right|>k^{1 / 5-10 \delta},
$$

where $m=j+n_{0} q, n_{0} \in Z,\left|n_{0}\right|>k^{1 / 5-9 \delta}$. This inequality is in contradiction with the assumption (5.28). Therefore, it remains to consider the case $|n|<$ $k^{1 / 5-9 \delta}$, i.e., $m \in \Pi_{q}\left(k^{1 / 5-9 \delta}\right)$. It is easy to see that $m \notin \Pi_{q}\left(k^{1 / 5-9 \delta}\right) \backslash T(k, \delta)$. Otherwise $P_{m m}=1$, but we consider inequality (5.27) in the case $i, m$ : $P_{m m}=P_{i i}=0$. Thus, $m \in T(k, \delta)$. Hence, inequality (3.12) holds. This inequality contradicts to (5.28). Thus, estimate (5.27) is valid. Taking into account that $R_{0}<k^{\delta / 8}$, we obtain

$$
\left\|A_{0}\right\|<k^{7 \delta} \text {. }
$$

We estimate $A_{1}^{(1)}$. Considering (5.2), we get:

$$
\left\|A_{1}^{(1)}\right\|^{2} \leq k^{1+\delta} \sum_{i}\left(\mathcal{E}_{q}\right)_{i j}^{2}\left|p_{i}^{2}+\Delta \hat{\Lambda}_{i i}-z\right|^{-1} .
$$

We prove the estimate

$$
3\left|p_{i}^{2}+\Delta \hat{\Lambda}_{i i}-z\right|>k^{1-11 \delta} .
$$

By (5.19) $\left(\mathcal{E}_{q}\right)_{i j}$ can differ from zero only if $i-j=l_{0} q+l q^{\prime}$, where $q^{\prime} \in \Gamma\left(R_{0}\right)$, $q^{\prime} \neq q,\left|l_{0}\right|<6 k^{1 / 5-9 \delta}, 1 \leq|l|<R_{0}$. First we estimate $\left|p_{i}^{2}(t)-p_{j}^{2}(t)\right|$. It is easy to see that

$$
\left|p_{i}^{2}(t)-p_{j}^{2}(t)\right|>
$$

$$
|| \vec{p}_{j}(t)+\left.l \vec{p}_{q^{\prime}}(0)\right|^{2}-p_{j}^{2}(t)|-2|\left(\vec{p}_{j}(t)+l \vec{p}_{q^{\prime}}(0), l \vec{p}_{q}(0)\right) \mid-l_{0}^{2} p_{q}^{2}(0) .
$$

Taking into account inequality (3.7) and the relation $\left|\left(\vec{p}_{j}(0), \vec{p}_{q}(0)\right)\right|<$ $k^{1 / 5-8 \delta}$, which holds because $j \in \Pi_{q}\left(k^{1 / 5-8 \delta}\right)$, we obtain

$$
\left|p_{i}^{2}(t)-p_{j}^{2}(t)\right|>k^{1-11 \delta} .
$$

Considering (5.2) and (5.34) we obtain (5.32). From estimates (5.31) and (5.32) taking into account (5.18), we get

$$
\left\|A_{1}^{(1)}\right\|<c k^{6 \delta} .
$$


Similarly we prove estimate

$$
\left\|A_{1}^{(2)}\right\|<c k^{6 \delta} .
$$

Adding estimates (5.30), (5.35), (5.36) and considering that $A_{1}^{(3)}=0$, we get (5.22).

2) Estimate (5.23) is proved similarly to the analogous estimate (5.51) in [1]. The difference is that we use conditions $3^{\prime}$ and $4^{\prime}$ instead of conditions $3^{0}$ and $4^{0}$ and we consider the operator $\left(I-P_{0}\right) \mathcal{E}_{q}\left(I-P_{0}\right)$ instead of $\mathcal{E}_{q}$ in the definition of $\varepsilon_{1 q}$.

3) We prove estimate (5.24). The proof up to some modifications is similar to the proof of relation (5.52) in [1]. In fact, first we verify that

$$
P_{k^{2}}\left(k^{1 / 5-8 \delta}\right) A_{1} P_{k^{2}}\left(k^{1 / 5-8 \delta}\right)=0 .
$$

We represent $A_{1}$ as the sum (5.11) and prove relation (5.37) for each of the operators $A_{0}, A_{1}^{(1)}, A_{1}^{(2)}\left(A_{1}^{(3)}=0\right)$. The relation

$$
P_{k^{2}}\left(k^{1 / 5-8 \delta}\right) A_{0} P_{k^{2}}\left(k^{1 / 5-8 \delta}\right)=0
$$

is proved just as the similar relation (5.54) in [1]. (instead of condition $5^{0}$ we use the condition $\left.5^{\prime}\right)$. The relation

$$
P_{k^{2}}\left(k^{1 / 5-8 \delta}\right) A_{1}^{(1)} P_{k^{2}}\left(k^{1 / 5-8 \delta}\right)=0 .
$$

easily follows from relation (5.20). Similarly,

$$
P_{k^{2}}\left(k^{1 / 5-8 \delta}\right) A_{1}^{(2)} P_{k^{2}}\left(k^{1 / 5-8 \delta}\right)=0 .
$$

Summing equalities (5.38)-(5.40), we get formula (5.37). Further, we represent $A_{1}$ in the form:

$$
M_{+}=P_{k^{2}}\left(k^{1 / 5-8 \delta}\right) A_{1}\left(I-P_{k^{2}}\left(k^{1 / 5-8 \delta}\right)\right)=P_{k^{2}}\left(k^{1 / 5-8 \delta}\right) A_{1},
$$

$$
M_{-}=\left(I-P_{k^{2}}\left(k^{1 / 5-8 \delta}\right)\right) A_{1} P_{k^{2}}\left(k^{1 / 5-8 \delta}\right)=A_{1} P_{k^{2}}\left(k^{1 / 5-8 \delta}\right) .
$$


Obviously,

$$
M_{+}^{2}=M_{-}^{2}=M_{0} M_{+}=M_{-} M_{0}=0 .
$$

From this,

$$
\begin{aligned}
A_{0}^{3}=M_{0}^{3}+M_{0}^{2} M_{-} & +M_{+} M_{0}^{2}+M_{0} M_{-} M_{+}+M_{+} M_{0} M_{+} M_{-} M_{+} M_{0} \\
& +M_{+} M_{-} M_{+}+M_{-} M_{+} M_{-} .
\end{aligned}
$$

Using the definition of $P_{k^{2}}\left(k^{1 / 5-8 \delta}\right)$ it is easy to see that

$$
\left\|M_{0}\right\|<k^{-1 / 5+9 \delta}, \quad\left\|M_{-}\right\|<k^{3 \delta},\left\|M_{+}\right\|<k^{3 \delta} .
$$

From (5.47) we obtain

$$
\begin{gathered}
\left\|M_{0}^{3}\right\|+\left\|M_{0}^{2} M_{-}\right\|+\left\|M_{+} M_{0}^{2}\right\|+\left\|M_{0} M_{-} M_{+}\right\|+ \\
\left\|M_{+} M_{0} M_{-}\right\|+\left\|M_{-} M_{+} M_{0}\right\|<k^{-1 / 5+15 \delta} .
\end{gathered}
$$

Assume that we have proved the inequality

$$
\left\|M_{+} M_{-}\right\|<k^{-1 / 5+26 \delta}
$$

Then, we have

$$
\left\|M_{+} M_{-} M_{+}\right\|,\left\|M_{-} M_{+} M_{-}\right\|<k^{-1 / 5+32 \delta},
$$

and summing the estimates (5.48) and (5.50), we get (5.24). Thus, to prove the necessary relation (5.24) it remains to check the estimate (5.49).

First, we consider the diagonal part of the operator $M_{+} M_{-}$:

$$
\left(M_{+} M_{-}\right)_{i i}=\sum_{|m|<R_{0}} \frac{\left|\left(B_{1}\right)_{i, i+m}\right|^{2}}{\left(p_{i}^{2}(t)+\Delta \hat{\Lambda}_{i i}-z\right)\left(p_{i+m}^{2}(t)+\Delta \hat{\Lambda}_{i+m, i+m}-z\right)},
$$

where

$$
B_{1}=P_{k^{2}}\left(k^{1 / 5-8 \delta}\right)\left(B_{0}+B_{1}^{(1)}+B_{1}^{(2)}\right)\left(I-P_{k^{2}}\left(k^{1 / 5-8 \delta}\right)\right) .
$$


Since $P_{0}\left(I-P_{k^{2}}\left(k^{1 / 5-8 \delta}\right)\right)=0$,

$$
B_{1}=P_{k^{2}}\left(k^{1 / 5-8 \delta}\right)\left(B_{0}+B_{1}^{(1)}\right)\left(I-P_{k^{2}}\left(k^{1 / 5-8 \delta}\right)\right) .
$$

Let us consider different $i$ in (5.51). If $i \neq j, i: P_{i i}=1$, then taking into account the definition of $B_{0}$ (formula (5.13)), we get $\left(M_{+} M_{-}\right)_{i i}=0$. The same is valid if $i: P_{k^{2}}\left(k^{1 / 5-9 \delta}\right)_{i i}=0$. It remains the case $i: P_{i i}=$ $0, P_{k^{2}}\left(k^{1 / 5-9 \delta}\right)_{i i}=1$. It is not hard to show (see Lemma 11 in [1] $\left(4^{0} \rightarrow 4^{\prime}\right)$ ) that formula

$$
\left(M_{+} M_{-}\right)_{i i}=\sum_{|m|<R_{0}} \frac{\left|v_{m}\right|^{2}\left(p_{i}^{2}(t)-z+p_{m}^{2}(0)\right)}{\left(p_{i}^{2}(t)-z\right)\left(p_{i+m}^{2}(t)-z\right)\left(p_{i-m}^{2}(t)-z\right)}
$$

holds. We prove that

$$
2\left|\left(M_{+} M_{-}\right)_{i i}\right|<k^{-1 / 5+25 \delta} .
$$

Let $i:\left|p_{i}^{2}(t)-k^{2}\right|>k^{-1 / 5-4 \delta}$. Considering the definitions of $M_{+}, M_{-}$and $P_{k^{2}}\left(k^{1 / 5-8 \delta}\right)$ we get (5.55). Suppose $i:\left|p_{i}^{2}(t)-k^{2}\right| \leq k^{-1 / 5-4 \delta}$. Considering as in the proof of (5.22), we obtain that

$$
36\left|\left(p_{i}^{2}(t)-z\right)\left(p_{i \pm m}^{2}(t)-z\right)\right|>k^{-4 \delta} .
$$

Using formula (5.54) and the last inequality, it is not hard to show that estimate (5.55) holds.

It remains only to consider the case $i=j$. It is clear that

$$
\left(M_{+} M_{-}\right)_{j j}=\sum_{|m|<R_{0}, m \neq 0} \frac{\left|\left(\mathcal{E}_{q}\right)_{j j+m}\right|^{2}}{\left(p_{j}^{2}(t)+\Delta \hat{\Lambda}_{j j}-z\right)\left(p_{j+m}^{2}(t)+\Delta \hat{\Lambda}_{j+m j+m}-z\right)} .
$$

According to (5.19) $\left(\mathcal{E}_{q}\right)_{j j+m}$ can differ from zero only if $m=l_{0} q+l q^{\prime}$, $\left|l_{0}\right|<k^{1 / 5}, q^{\prime} \in \Gamma\left(R_{0}\right), q^{\prime} \neq q, 1 \leq|l|<R_{0}$. From this we obtain

$$
p_{j+m}^{2}(t)+\Delta \hat{\Lambda}_{j+m, j+m}-z=2 l\left(\vec{p}_{j}(0), \vec{p}_{q^{\prime}}(0)\right)+O\left(k^{2 / 5}\right) .
$$

From relation (3.7) it follows

$$
8\left|\left(\vec{p}_{j}(0), \vec{p}_{q^{\prime}}(0)\right)\right|>k^{1-10 \delta} .
$$


Let

$$
\begin{gathered}
a_{l}=\sum_{m \in \Omega_{l q^{\prime}}}\left|\left(\mathcal{E}_{q}\right)_{j j+m}\right|^{2}, \\
\Omega_{l q^{\prime}}=\left\{m: \vec{p}_{m}(0)=l_{0} q+l q^{\prime}\right\} .
\end{gathered}
$$

Using relations (5.57)-(5.59), it is not hard to show that

$$
\left(M_{+} M_{-}\right)_{j j}=\left(\sum_{l} a_{l}(2 l)^{-1}\right)\left(p_{j}^{2}(t)+\Delta \Lambda_{j j}-z\right)^{-1}\left|\left(\vec{p}_{j}(0), \vec{p}_{q^{\prime}}(0)\right)\right|^{-1}+O\left(k^{-3 / 5+20 \delta}\right.
$$

Using again relations (5.2) and (5.59) we get

$$
\left(M_{+} M_{-}\right)_{j j}=\left(\sum_{l q} a_{l}(2 l)^{-1}\right) O\left(k^{11 \delta}\right)+O\left(k^{-3 / 5+20 \delta}\right) .
$$

We prove that $a_{l}=a_{-l}$. Indeed, recalling the definition of $\mathcal{E}_{q}$ (see [1]), we verify that

$$
a_{l}=\sum_{l_{1}, l_{2}}\left|U_{j, j+l_{1} q} v_{l q^{\prime}} U_{j+l_{1} q+l q^{\prime}+l_{2} q}^{*}\right|^{2}
$$

where $U$ is the unitary operator reducing $\hat{H}$ to the diagonal form. Summing with respect to indexes $l_{1}$ and $l_{2}$, we get:

$$
a_{l}=\left|v_{l q^{\prime}}\right|^{2}=a_{-l} .
$$

Now we see that $\sum_{l} a_{l}(2 l)^{-1}=0$. Therefore,

$$
\left|\left(M_{+} M_{-}\right)_{j j}\right| \leq c k^{-3 / 5+20 \delta} .
$$

Next, we consider non-diagonal elements: $\left(M_{+} M_{-}\right)_{i l}, i \neq l$,

$$
\left(M_{+} M_{-}\right)_{i l}=
$$$$
\sum_{m} \frac{\left(B_{1}\right)_{i m}\left(B_{1}\right)_{m l}}{\left(p_{i}^{2}(t)+\Delta \hat{\Lambda}_{i i}(t)-z\right)^{1 / 2}\left(p_{l}^{2}(t)+\Delta \hat{\Lambda}_{l l}(t)-z\right)^{1 / 2}\left(p_{m}^{2}(t)+\Delta \hat{\Lambda}_{m m}(t)-z\right)}
$$

where

$$
P_{k^{2}}\left(k^{1 / 5-9 \delta}\right)_{i i}=P_{k^{2}}\left(k^{1 / 5-9 \delta}\right)_{l l}=1
$$




$$
P_{k^{2}}\left(k^{1 / 5-9 \delta}\right)_{m m}=0 .
$$

From the last relation it follows that $m \neq j$. Suppose $i=j$, then $l \neq j$ (because we consider non-diagonal elements). It is clear that $j-l=l_{0} q+$ $l q^{\prime},\left|l_{0}\right|<k^{1 / 5-8 \delta}, 1 \leq\left|l_{1}\right|<R_{0}, l_{1} \neq 0$. Since $\left|\left(\vec{p}_{j}(0), \vec{p}_{q^{\prime}}(0)\right)\right|>k^{3 / 5}$, $\left|\left(\vec{p}_{j}(0), \vec{p}_{q}(0)\right)\right|<6 k^{1 / 5-8 \delta}$, we readily obtain

$$
\left|p_{l}^{2}(t)-k^{2}\right|=\left|p_{l}^{2}(t)-p_{j}^{2}(t)\right|>k^{3 / 5-\delta} .
$$

This inequality contradicts relation (5.66). Therefore $i \neq j$. Similarly, we obtain that $l \neq j$, otherwise $\left(M_{+} M_{-}\right)_{i l}=0$ (for $\left.i \neq l\right)$. Since

$$
\left(B_{1}\right)_{i m}=\left(B_{0}\right)_{i m}
$$

for $i \neq j, m \neq j$, we have

$$
\left(M_{+} M_{-}\right)_{i l}=\sum_{m} \frac{\left(B_{0}\right)_{i m}\left(B_{0}\right)_{m l}}{\left(p_{i}^{2}(t)-z\right)^{1 / 2}\left(p_{l}^{2}(t)-z\right)^{1 / 2}\left(p_{m}^{2}(t)-z\right)},
$$

Using relation (5.27), which is valid for all $i, m:|i-m|<k^{\delta}$, we get

$$
6\left|\left(p_{i}^{2}(t)-z\right)\left(p_{l}^{2}(t)-z\right)\right|^{1 / 2}>k^{-2 \delta} \text {. }
$$

Taking into account that $\left|p_{m}^{2}(t)-k^{2}\right|>k^{1 / 5-8 \delta}$, (see (5.67) we arrive at the estimate

$$
\left|\left(M_{+} M_{-}\right)_{i l}\right|<k^{-1 / 5+10 \delta}, \quad i \neq l .
$$

Since $\left(M_{+} M_{-}\right)_{i l}=0$ if $|i-l|>2 R_{0}$, it follows that

$$
\left\|M_{+} M_{-}\right\|<k^{\delta} \max _{i, j}\left|\left(M_{+} M_{-}\right)_{i j}\right| .
$$

Taking into account (5.55), (5.64) and (5.72), we get (5.49). Thus, estimate (5.10) is proved. The lemma is proved.

Lemma 5.3. If $t$ is in the $\left(k^{-2-2 \delta}\right)$-neighborhood of the set $\hat{\chi}_{q}^{0}(k, V, \delta)$ and $z \in C_{1}$, then for sufficiently large $k, k>k_{0}(V, \delta)$ the following estimates hold:

$$
\begin{gathered}
\left\|\hat{G}_{1}^{\prime}(k, t)\right\|_{1}<k^{-1+4 \delta} \\
\left\|\hat{G}_{2}^{\prime}(k, t)\right\|_{1}<k^{-7 / 5+6 \delta} \\
\left|\hat{g}_{2}^{\prime}(k, t)\right|<k^{-8 / 5+8 \delta}
\end{gathered}
$$


Proof. Evaluating the integral $U \hat{G}_{1}^{\prime}(k, t) U^{*}(U$ is the unitary operator, reducing $\hat{H}(t)$ to the diagonal form) by a residue, we obtain $\left(U \hat{G}_{1}^{\prime}(k, t) U^{*}\right)_{i m}=$ 0 , if $i \neq j, m \neq j$ or $i=m=j$ and

$$
\left(U \hat{G}_{1}^{\prime}(k, t) U^{*}\right)_{j i}=\overline{\left.\left(U \hat{G}_{1}^{\prime}(k, t) U^{*}\right)_{i j}\right)}=
$$

$$
B_{j i}\left(p_{i}^{2}(t)+\Delta \hat{\Lambda}_{i i}(t)-p_{j}^{2}(t)-\Delta \hat{\Lambda}_{j j}(t)\right)^{-1},
$$

where $B=B_{0}+\sum_{q} \mathcal{E}_{q}+L+D$, operators $L$ and $D$ being described in [1]. Here we only will use their properties. Taking into account that $\left(B_{0}\right)_{j i}=$ $L_{j i}=0\left(j \in \Pi_{q}\left(k^{\delta}\right)\right)$ and $\left(\mathcal{E}_{q^{\prime}}\right)_{j i}=0$ if $q^{\prime} \neq q$, we get

$$
B_{j i}=\left(\mathcal{E}_{q}\right)_{j i}+D_{j i}
$$

From (5.77), using (5.76), we obtain

$$
\left|\left(U \hat{G}_{1}^{\prime}(k, t) U^{*}\right)_{j i}\right|<a_{i}+c_{i}
$$

where

$$
\begin{gathered}
a_{i}=\left|\left(\mathcal{E}_{q}\right)_{j i}\right|\left|p_{i}^{2}(t)+\Delta \hat{\Lambda}_{i i}(t)-p_{j}^{2}(t)-\Delta \hat{\Lambda}_{j j}(t)\right|^{-1}, \\
c_{i}=\left|D_{j i}\right|\left|p_{i}^{2}(t)+\Delta \hat{\Lambda}_{i i}(t)-p_{j}^{2}(t)-\Delta \hat{\Lambda}_{j j}(t)\right|^{-1}
\end{gathered}
$$

Terms similar to $a_{i}$ were estimated in the proof of Lemma 4 (see formulae (5.31)-(5.36)). Using estimate (5.32) and (5.2) we get

$$
2 a_{i}<\left|\left(\mathcal{E}_{q}\right)_{j i}\right| k^{-1+11 \delta} .
$$

Relations (5.1) and (5.2) yield

$$
c_{i}<\left|D_{j i}\right| k^{1+\delta} .
$$

It is clear that

$$
\left\|\hat{G}_{1}^{\prime}(k, t)\right\|_{1}^{2}=\left\|U \hat{G}_{1}^{\prime}(k, t) U^{*}\right\|_{1}^{2} \leq \sum_{i}\left|\left(U \hat{G}_{1}^{\prime}(k, t) U^{*}\right)_{j i}\right|^{2} .
$$

Considering estimates (5.78)-(5.80) we get

$$
\left\|\hat{G}_{1}^{\prime}(k, t)\right\|_{1} \leq\left\|\mathcal{E}_{q}\right\| k^{-1+3 \delta}+\|D\| k^{1+\delta} .
$$

Taking into account that

$$
\left\|\mathcal{E}_{q}\right\|<\|V\|
$$




$$
\|D\|<k^{-k^{1 / 5-86}}
$$

(see cite1), we arrive at estimate (5.73).

Next, we consider $\left\|\hat{G}_{2}^{\prime}(k, t)\right\|_{1}$. Evaluating the integral by a residue, we get

$$
\left(U \hat{G}_{2}^{\prime}(k, t) U^{*}\right)_{i m}=\left(U \hat{G}_{1}^{\prime}(k, t) U^{*}\right)_{i j}\left(U \hat{G}_{1}^{\prime}(k, t) U^{*}\right)_{j m},
$$

when $i \neq j, m \neq j$ and

$$
\begin{gathered}
\left(U \hat{G}_{2}^{\prime}(k, t) U^{*}\right)_{j m}=\overline{\left.\left(U \hat{G}_{2}^{\prime}(k, t) U^{*}\right)_{m j}\right)}=\tilde{a}_{m}+\tilde{c}_{m}, \\
\tilde{a}_{m}=-B_{j j} B_{j m}\left(p_{m}^{2}(t)+\Delta \hat{\Lambda}_{m m}(t)-p_{j}^{2}(t)-\Delta \hat{\Lambda}_{j j}(t)\right)^{-2}+ \\
\tilde{c}_{m}=
\end{gathered}
$$

$\sum_{i \neq j} B_{j i} B_{i m}\left(p_{i}^{2}(t)+\Delta \hat{\Lambda}_{i i}(t)-p_{j}^{2}(t)-\Delta \hat{\Lambda}_{j j}(t)\right)^{-1}\left(p_{m}^{2}(t)+\Delta \hat{\Lambda}_{m m}(t)-p_{j}^{2}(t)-\Delta \hat{\Lambda}_{j j}(t)\right)^{-1}$

when $m \neq j$ and

$$
\left(U \hat{G}_{2}^{\prime}(k, t) U^{*}\right)_{j j}=-\sum_{m \neq j}\left|B_{j m}\right|^{2}\left(p_{m}^{2}(t)+\Delta \hat{\Lambda}_{m m}(t)-p_{j}^{2}(t)-\Delta \hat{\Lambda}_{j j}(t)\right)^{-2} .
$$

From relations (5.80)-(5.85) we see

$$
\begin{gathered}
\left\|\hat{G}_{2}^{\prime}(k, t)\right\|_{1}^{2} \leq\left\|\hat{G}_{1}^{\prime}(k, t)\right\|_{1}^{2}+2 I, \\
I=\sum_{m}\left|\left(U \hat{G}_{2}^{\prime}(k, t) U^{*}\right)_{j m}\right|^{2} .
\end{gathered}
$$

To estimate $I$ we represent it as a sum $I=I_{1}+I_{2}+I_{3}$, where $I_{k}$ corresponds to the summation over the set $\Omega_{k}$ :

$$
\begin{gathered}
\Omega_{1}=\left\{m: m \in \Pi_{q}\left(\frac{1}{2} k^{1 / 5-8 \delta}\right)\right\}, \\
\Omega_{2}=\left\{m: m \in \Pi_{q}\left(\frac{3}{2} k^{1 / 5-8 \delta}\right) \backslash \Pi_{q}\left(\frac{1}{2} k^{1 / 5-8 \delta}\right)\right\}, \\
\Omega_{3}=\left\{m: m \notin \Pi_{q}\left(\frac{3}{2} k^{1 / 5-8 \delta}\right)\right\} .
\end{gathered}
$$


Suppose $m \in \Omega_{1}$. Then, $L_{i m}=\left(B_{0}\right)_{i m}=0$ and, therefore,

$$
B_{i m}=\left(\mathcal{E}_{q}\right)_{i m}+D_{i m}
$$

Considering as in the proof of (5.73), we obtain

$$
I_{1}<c k^{-2+8 \delta} \text {. }
$$

Suppose $m \in \Omega_{2}$. Using (5.84) we get

$$
\sum_{m \in \Omega_{2}}\left|\left(U \hat{G}_{2}^{\prime}(k, t) U^{*}\right)_{j m}\right|^{2}<\sum_{m \in \Omega_{2}} \tilde{a}_{m}+\sum_{m \in \Omega_{2}} \tilde{c}_{m} .
$$

To estimate $\tilde{a}_{m}$ note that $B_{j m}$ is given by formula (5.77). Considering as in the proof of (5.73) we get

$$
\sum_{m \in \Omega_{2}} \tilde{a}_{m}<k^{-2+8 \delta}
$$

To estimate $\tilde{c}_{m}$ we recall [1] that for any $m \in \Omega_{2}$

$$
\left|p_{m}^{2}(t)+\Delta \hat{\Lambda}_{m m}(t)-p_{j}^{2}(t)-\Delta \hat{\Lambda}_{j j}(t)\right|>c k^{2 / 5} .
$$

Using this inequality and (5.32) we get

$$
\sum_{m \in \Omega_{2}} \tilde{c}_{m}<k^{-7 / 5+8 \delta}
$$

Thus,

$$
I_{2}<c k^{-7 / 5+8 \delta} .
$$

If $m \in \Omega_{3}$, then in formula (5.84) $B_{i m}=\left(B_{0}\right)_{i m}$. This means that $|i-m|<R_{0}$. On the other hand $B_{j i}$ is given by (5.77). We consider that $\vec{p}_{j}(0)-\vec{p}_{i}(0)=l_{0} \vec{p}_{q}(0)+l \vec{p}_{q^{\prime}}(0)$, where $\left|l_{0}\right|<k^{1 / 5-8 \delta}, 1 \leq$ $|l|<R_{0}$, because otherwise $\left(\mathcal{E}_{q}\right)_{j i}=0$ while $D_{j i}$ is estimated by tiny value $k^{-k^{1 / 5-86}}$. Therefore, $\vec{p}_{j}(0)-\vec{p}_{m}(0)=l_{1} \vec{p}_{q}(0)+l_{2} \vec{p}_{q^{\prime \prime}}(0)$, where $\left|l_{1}\right|<k^{1 / 5-8 \delta},\left|l_{2}\right|<2 R_{0}, l_{2} \vec{p}_{q^{\prime \prime}}(0) \neq 0$ (the last relation holds because otherwise $\left.m \in \Pi_{q}\left(3 k^{1 / 5-8 \delta} / 2\right) \not \subset \Omega_{3}\right)$. Considering as in the proof of (5.73) we show that

$$
\begin{gathered}
\left|p_{i}^{2}(t)+\Delta \hat{\Lambda}_{i i}(t)-p_{j}^{2}(t)-\Delta \hat{\Lambda}_{j j}(t)\right|>c k^{1-8 \delta} \\
\left|p_{m}^{2}(t)+\Delta \hat{\Lambda}_{m m}(t)-p_{j}^{2}(t)-\Delta \hat{\Lambda}_{j j}(t)\right|>c k^{1-8 \delta}
\end{gathered}
$$


Using this inequality and the estimate $\|B\| \leq\|V\|$, we get

$$
I_{3}<c k^{-2+8 \delta} \text {. }
$$

Adding estimates (5.88), (5.89) and (5.90), we get

$$
I<c k^{-7 / 5+8 \delta} .
$$

Using formula (5.86) and estimates (5.73), (5.91), we obtain (5.74).

Inequality $(5.75)$ we obtain, calculating $\hat{g}_{2}^{\prime}(k, t)$ by a residue:

$$
\hat{g}_{2}^{\prime}(k, t)=\sum_{m \neq j}\left|B_{j m}\right|^{2}\left(p_{m}^{2}(t)+\Delta \hat{\Lambda}_{m m}(t)-p_{j}^{2}(t)-\Delta \hat{\Lambda}_{j j}(t)\right)^{-1} .
$$

Using formula (5.77) and the inequalities

$$
\begin{gathered}
\|D\|<k^{-k^{1 / 5-8 \delta}}, \\
2\left|p_{m}^{2}(t)+\Delta \hat{\Lambda}_{m m}(t)-p_{j}^{2}(t)-\Delta \hat{\Lambda}_{j j}(t)\right|>k^{-1-\delta}, \quad m \neq j
\end{gathered}
$$

(see inequalities (3.10) and (3.11)), we obtain

$$
\hat{g}_{2}^{\prime}(k, t)=\sum_{m \neq j}\left|\left(\mathcal{E}_{q}\right)_{j m}\right|^{2}\left(p_{m}^{2}(t)+\Delta \hat{\Lambda}_{m m}(t)-p_{j}^{2}(t)-\Delta \hat{\Lambda}_{j j}(t)\right)^{-1}+O\left(k^{-2}\right) .
$$

From the last relation, (5.57) and (5.2) it is easy to see that $\left|\hat{g}_{2}^{\prime}(k, t)\right|=$ $\left|\left(M_{+} M_{-}\right)_{j j}\right| k^{-1-\delta}+O\left(k^{-2}\right)$. Using (5.64), we get estimate(5.75).

The lemma is proved.

The proofs of Theorem 3 and 4 are quite similar to the proofs of Theorems 1 and 2 in [1], Lemma 4 being using instead of Lemma 11 in [1].

Next, we consider the behaviour of the isoenergetic surface in a vicinity of the singular set. Since the eigenvalues and the spectral projections of operator $H$ are close to those of $\hat{H}$ on the essential part of the singular set, the isoenergetic surface of $H$ is close to that of $\hat{H}$.

According to Theorem 3 there exists the subset $\hat{\chi}_{q}^{0}(k, V, \delta)$ of $\hat{\mu}_{q}(k, \delta)$ on which the perturbation series converge. This subset has an asymptotically full measure on $\hat{\mu}_{q}(k, \delta)$. We denote by $\hat{\chi}_{q}^{0}(k, V, \delta)_{j}$ the intersection of $\hat{\chi}_{q}^{0}(k, V, \delta)$ with $\hat{\mu}_{q}(k, \delta)_{j}$ (see (4.1)).

Lemma 5.4. In the $\left(k^{-2-2 \delta}\right)$-neighbourhood of a piece $\hat{\chi}_{q}^{0}(k, V, \delta)_{j}$ of the isoenergetic surface there are no other ones $\hat{\chi}_{q}^{0}(k, V, \delta)_{i}, i \neq j$. 
Proof. Suppose, that in the $\left(k^{-2-2 \delta}\right)$-neighbourhood of point $t, t \in$ $\hat{\chi}_{q}^{0}(k, V, \delta)_{j}$ there exists point $t_{0}, t_{0} \in \hat{\chi}_{q}^{0}(k, V, \delta)_{i}, i \neq j$. Then,

$$
\begin{gathered}
p_{j}^{2}(t)+\hat{\Delta} \Lambda(t)_{j j}=k^{2}, \quad p_{i}^{2}\left(t_{0}\right)+\hat{\Delta} \Lambda\left(t_{0}\right)_{i i}=k^{2}, \\
\left|t-t_{0}\right|<k^{-2-2 \delta} .
\end{gathered}
$$

It is obvious that

$$
\left|p_{i}^{2}(t)+\hat{\Delta} \Lambda(t)_{i i}-p_{i}^{2}\left(t_{0}\right)+\hat{\Delta} \Lambda\left(t_{0}\right)_{i i}\right|<k^{-1-2 \delta} .
$$

Therefore

$$
\left|p_{j}^{2}(t)+\hat{\Delta} \Lambda(t)_{j j}-p_{i}^{2}(t)+\hat{\Delta} \Lambda(t)_{i i}\right|<k^{-1-2 \delta} .
$$

However, it can not be so, because $t \in \hat{\chi}_{q}^{0}(k, V, \delta)$ (see inequalities (3.10), (3.11)).

The lemma is proved.

The proof of Theorem 7. Suppose $t$ belongs to the $\left(k^{-2-2 \delta}\right)$-neighbourhood of $\hat{\chi}_{q}^{0}(k, V, \delta)$. According to Corollaries 2 and 3 the following estimates hold:

$$
\begin{gathered}
\left|\hat{\lambda}(t)-p_{j}^{2}(t)-\Delta \Lambda(t)_{j j}\right|<c k^{-1-2 \gamma_{2}}, \\
\left|\nabla \hat{\lambda}(t)-\nabla\left(p_{j}^{2}(t)-\Delta \Lambda(t)_{j j}\right)\right|<c k^{1-2 \gamma_{2}+\delta} .
\end{gathered}
$$

From the last relation it follows

$$
|\nabla \hat{\lambda}(t)|>c k .
$$

Using estimates (5.95) and (5.97) we obtain that equation (4.3) has a solution in the $\left(k^{-2-2 \gamma_{1}}\right)$-neighbourhood of any point $t_{0}$, belonging to $\hat{\chi}_{q}^{0}(k, V, \delta)$. These solutions form a surface in the $\left(k^{-2-2 \gamma_{1}}\right)$-neighbourhood of $\hat{\chi}_{q}^{0}(k, V, \delta)$, because (5.96) holds.

Next, we prove that equation (4.3) has no other solutions in the $\left(k^{-2-2 \delta}\right)$-neighbourhood of $\hat{\chi}_{q}^{0}(k, V, \delta)$. Indeed, suppose $\tilde{t}$ is in the $\left(k^{-2-2 \delta}\right)$ neighbourhood of $\hat{\chi}_{q}^{0}(k, V, \delta)$ and there exists an eigenvalue $\tilde{\lambda}(\tilde{t})$ of operator $H$, which cannot be represented as the series (3.16), i.e., $\tilde{\lambda}(\tilde{t}) \neq$ $\hat{\lambda}(\tilde{t})$. According to Theorem $4 \hat{\lambda}(\tilde{t})$ is a unique eigenvalue in the interval $\left(k^{2}-k^{-1-1 \delta}, k^{2}+k^{-1-\delta}\right)$. However, $\tilde{\lambda}(\tilde{t})=k^{2}$. From this it follows that $\tilde{\lambda}(\tilde{t})=\hat{\lambda}(\tilde{t})$. The theorem is proved. 


\section{Geometric Constructions on the Singular Set.}

We construct now the subset $\hat{\chi}_{q}^{0}(k, V, \delta)$ of $\hat{\mu}_{q}(k, \delta)$ on which conditions $1^{\prime}--5^{\prime}$ hold. The analytical description of $1^{\prime}--5^{\prime}$ is similar to that of $1^{0}--5^{0}$ for the nonsingular set. Howver, to prove that $1^{\prime}--5^{\prime}$ in fact can be satisfied, we have to develop much more subtle reasoning than in [1]. This is due to the fact that conditions $1^{0}--5^{0}$ were proved to be satisfied for some $t$ on $\mu_{q}$ which is, as a matter of fact, only a small part of $S_{k}$. So more precise estimates and complicated reasoning have to be done. We proved that the nonsingular set has an asymptotically full measure on $S_{k}$. Here we will prove that $\hat{\chi}_{q}^{0}(k, V, \delta)$ has an asymptotically full measure on $\hat{\mu}_{q}(k, \delta)$.

We consider

$$
\hat{\chi}_{q}^{0}(k, V, \delta) \equiv \hat{\mu}_{q}(k, \delta) \backslash \cup_{i=1}^{5} \hat{\Upsilon}_{q}(k, V, \delta)_{i},
$$

where the set $\hat{\Upsilon}_{q}(k, V, \delta)_{i}$ contains all points $t$, for which condition $i^{\prime}$ (see Lemma 1) does not hold. These sets will be described below. The definition of the set $\hat{\chi}_{q}^{0}(k, V, \delta)$ is similar to the definition of the nonsingular set $S(k, V, \delta)$ in [1], where conditions $1^{0}-5^{0}$ are satisfied.

First, we introduce the notations. Let $\Pi_{m}(k, a)$ be the plane layer:

$$
\Pi_{m}(k, a)=\left\{x:\left.|| x\right|^{2}-\left|x+\vec{p}_{m}(0)\right|^{2} \mid<4 k^{-a}\right\}
$$

Let $\hat{\Pi}_{m}(k, a)$ be the curve analog of the plane layer $\Pi_{m}(k, a)$ :

$$
\hat{\Pi}_{m}(k, a)=\left\{x:\left.|| x\right|^{2}+\varphi_{0}(x)-\left|x+\vec{p}_{m}(0)\right|^{2} \mid<4 k^{-a}\right\},
$$

where the function $\varphi_{0}(x)$ is given by formula (3.5).

We determine $\hat{\Upsilon}_{q}(k, V, \delta)_{1}$ by the formula:

$$
\hat{\Upsilon}_{q}(k, V, \delta)_{1}=\mathcal{K}\left(\cup_{r \in \Omega_{1}} \hat{\Pi}_{r}(k,-1+10 \delta) \cap \hat{S}_{q}(k,-\delta)\right),
$$

$\Omega_{1}$ is given by (3.8), $\mathcal{K}$ is defined by (2.4). Next,

$$
\begin{gathered}
\hat{\Upsilon}_{q}(k, V, \delta)_{2}=\mathcal{K}\left(\cup_{m \in \Omega_{2}, i \in \Omega_{3}} \hat{\Upsilon}_{q}(k, V, \delta)_{2}^{m i} \cap \hat{S}_{q}(k,-\delta)\right) \\
\Omega_{2}=\left\{m, m \in Z^{3}, m \neq n_{0} q, n_{0} \in Z\right\} \\
\Omega_{3}=\left\{i, i \in Z^{3}, i \neq 0,|i|<k^{\delta}\right\} \\
\hat{\Upsilon}_{q}(k, V, \delta)_{2}^{m i}=\cup_{n=0}^{N} \hat{\Upsilon}_{q}(k, V, \delta)_{2}^{m i n}, \quad N=[1 / \delta]-19
\end{gathered}
$$




$$
\hat{\Upsilon}_{q}(k, V, \delta)_{2}^{m i n}=\hat{\Pi}_{m}(k, V, 1-\delta n) \cap \hat{\Pi}_{m+i}(k, V,-1+\delta(n+11)) .
$$

Furthermore, let

$$
\begin{gathered}
\hat{\Upsilon}_{q}(k, V, \delta)_{3}=\mathcal{K}\left(\cup_{m \in \Omega_{2}} \hat{\Pi}_{m}(k,-1+\delta) \cap \hat{S}_{q}(k,-\delta)\right), \\
\hat{\Upsilon}_{q}(k, V, \delta)_{4}=\cup_{q^{\prime} \in \Gamma\left(R_{0}\right)} \hat{\Upsilon}_{q}(k, V, \delta)_{4 q^{\prime}},
\end{gathered}
$$

where $\hat{\Upsilon}_{q}(k, V, \delta)_{4 q^{\prime}}$ is set of $t$ in $\hat{\mu}_{q}(k, \delta)$ such that

$$
\left|p_{m}^{2}(t)+\Delta \hat{\Lambda}_{m m}(t)-p_{j}^{2}(t)-\Delta \hat{\Lambda}_{j j}(t)\right|<k^{-1 / 5-5 \delta}
$$

for any $m$ of $\Pi_{q^{\prime}}\left(k^{1 / 5}\right)$. This means that $\hat{\Upsilon}_{q}(k, V, \delta)_{4 q^{\prime}}$ the eigenvalues $p_{m}^{2}(t)+$ $\Delta \hat{\Lambda}_{m m}(t)$ are situated rather close to the point $p_{j}^{2}(t)+\Delta \hat{\Lambda}_{j j}(t)=k^{2}$. Finally,

$$
\hat{\Upsilon}_{q}(k, \delta)_{5}=\mathcal{K} \hat{\pi}(k, 1 / 5,3 / 5,1 / 5-8 \delta),
$$

where $\hat{\pi}$ is determined by the formula:

$$
\begin{gathered}
\hat{\pi}=\cup_{q, q^{\prime} \in \Gamma\left(R_{0}\right), q \neq q^{\prime}} \hat{\pi}_{q q^{\prime}}, \\
\hat{\pi}_{q q^{\prime}}=\left\{x, x \in R^{3}:|| x|-k|<k^{1 / 5},\right. \\
\left.\left|\left(x, \vec{p}_{q}(0)\right)\right|<2 k^{1 / 5-8 \delta},\left|\left(x, \vec{p}_{q^{\prime}}(0)\right)\right|<k^{3 / 5}\right\} .
\end{gathered}
$$

Lemma 6.1. If $t$ belongs to $\hat{\chi}_{q}^{0}(k, V, \delta)$, then there exists a unique $j$, such that $p_{j}^{2}(t)+\Delta \hat{\Lambda}_{j j}(t)=k^{2}$ and conditions $1^{\prime}-5^{\prime}$ hold. For $t$ in the $\left(k^{-2-2 \delta}\right)$-neighbourhood of $\hat{\chi}_{q}^{0}(k, V, \delta)$ there exists a unique $j$ such that $\left|p_{j}^{2}(t)+\Delta \hat{\Lambda}_{j j}(t)-k^{2}\right|<k^{-1-2 \delta}$, and conditions $1^{\prime}-5^{\prime}$ hold.

Proof. Since $t \in \hat{\mu}_{q}(k, \delta)$, there exist at least one $j$ such that $\vec{p}_{j}(t) \in$ $\hat{S}_{q}(k,-\delta)$, i.e., $p_{j}^{2}(t)+\Delta \hat{\Lambda}_{j j}(t)=k^{2}$, and $\left|p_{j}^{2}(t)-p_{j+q}(t)^{2}\right|<k^{\delta}$. Suppose, inequality (3.7) does not hold. Then for $x=\vec{p}_{j}(t)$ and some $r \in \Omega_{1}$ :

$$
\left.|| x\right|^{2}+\varphi_{0}(x)-\left|x+\vec{p}_{m}(0)\right|^{2} \mid<k^{1-10 \delta} .
$$

Thus, we have $x \in \hat{\Pi}_{m}(k,-1+10 \delta) \cap \hat{S}_{q}(k,-\delta)$. Therefore, $t \in \mathcal{K}\left(\hat{\Pi}_{m} \cap\right.$ $\left.\hat{S}_{q}\right) \subset \hat{\Upsilon}_{q}(k, V, \delta)_{1}$. However, $\hat{\chi}_{q}^{0}(k, V, \delta) \cap \hat{\Upsilon}_{q}(k, V, \delta)_{1}=\emptyset$ (see (6.4)). This contradiction proves inequality (3.7). 
Next, suppose condition $3^{\prime}$ does not hold. This means that there exists $m$ such that $m \neq n_{0} q,\left(n_{0} \in Z\right)$ and

$$
\left|p_{m+j}^{2}(t)-p_{j}^{2}(t)-\Delta \hat{\Lambda}(t)_{j j}\right|<2 k^{-1-\delta} .
$$

The last inequality means that for $x=\vec{p}_{j}(t)$ the next relation is valid:

$$
\left.|| x\right|^{2}+\varphi_{0}(x)-\left|x+\vec{p}_{m}(t)\right|^{2} \mid<2 k^{-1-\delta},
$$

i.e., $x \in \hat{\Pi}_{m}(k, 1+\delta) \cap \hat{S}_{q}(k,-\delta)$. Therefore $t \in \mathcal{K}\left(\hat{\Pi}_{m}(k, 1+\delta) \cap \hat{S}_{q}(k,-\delta)\right) \subset$ $\hat{\Upsilon}_{q}(k, V, \delta)_{3}$. However, $\hat{\chi}_{q}^{0}(k, V, \delta) \cap \hat{\Upsilon}_{q}(k, V, \delta)_{3}=\emptyset$. This contradiction proves that condition $3^{\prime}$ holds.

Suppose condition $2^{\prime}$ does not hold. This means that there exists $m, m \neq$ $n_{0} q\left(n_{0} \in Z\right)$, such that

$$
\left|p_{m+j}^{2}(t)-p_{j}^{2}(t)-\Delta \hat{\Lambda}(t)_{j j}\right|<k^{-20 \delta} .
$$

Additionally, there exist some $i,|i|<k^{\delta}, i \neq 0$, such that the inequality opposite to (3.9) is satisfied:

$$
\left|p_{m+j+i}^{2}(t)-p_{j}^{2}(t)-\Delta \hat{\Lambda}(t)_{j j}\right| \leq k^{-12 \delta}\left|p_{m+j}^{2}(t)-p_{j}^{2}(t)-\Delta \hat{\Lambda}(t)_{j j}\right|^{-1} .
$$

Inequalities (6.17) and (3.10) mean that

$$
\vec{p}_{j}(t) \in\left(\cup_{n=0}^{N} \hat{\Pi}_{m}(k, 1-\delta n)\right) \backslash \hat{\Pi}_{m}(k, 1+\delta), N=[1 / \delta]-19 .
$$

The sets $\hat{\Pi}_{m}(k, 1-\delta n), n=0, \ldots, N$, form a sequence of expanding sets. It is clear that

$$
\vec{p}_{j}(t) \in \hat{\Pi}_{m}(k, 1-\delta n) \backslash \hat{\Pi}_{m}(k, 1-\delta(n-1))
$$

for some $n \in\{0, \ldots, N\}$. Relation (6.18) means that

$$
\left|p_{m+i+j}^{2}(t)-p_{j}^{2}(t)-\Delta \hat{\Lambda}(t)_{j j}\right|<k^{1-\delta n-11 \delta} .
$$

i.e., $\vec{p}_{j}(t) \in \Pi_{i+m}(k,-1+\delta n+11 \delta)$. Using relation (6.20) and formula (6.8), we get

$$
\vec{p}_{j}(t) \in \hat{\Upsilon}_{q}(k, V, \delta)_{2}^{m i n} \subset \hat{\Upsilon}_{q}(k, V, \delta)_{2}^{m i} .
$$

Therefore, $t \in \hat{\Upsilon}_{q}(k, V, \delta)_{2}$. However, $\hat{\Upsilon}_{q}(k, V, \delta)_{2} \cap \hat{\chi}_{q}^{0}(k, V, \delta)=\emptyset$. This contradiction proves that condition $2^{\prime}$ is satisfied. 
Suppose, condition $4^{\prime}$ does not hold. Therefore, there exists $m \in \Pi_{0}(k)$, $\Pi_{0}=\cup_{q^{\prime} \in \Gamma\left(R_{0}\right)} \Pi_{q^{\prime}}\left(k^{1 / 5}\right)$ such that

$$
\left|p_{m}^{2}(t)+\Delta \hat{\Lambda}(t)_{m m}-p_{j}^{2}(t)-\Delta \hat{\Lambda}(t)_{j j}\right|<k^{-1 / 5-10 \delta},
$$

i.e., $t \in \hat{\Upsilon}_{q}(k, V, \delta)_{4}$. Since $\hat{\chi}_{q}(k, V, \delta) \cap \hat{\Upsilon}_{q}(k, V, \delta)_{4}=\emptyset$, condition $4^{\prime}$ holds.

Next, suppose condition $5^{\prime}$ is not satisfied. This means that there exists $m \in T(k, \delta)$ such that

$$
\left|p_{m}^{2}(t)-p_{j}^{2}(t)-\Delta \hat{\Lambda}(t)_{j j}\right|<k^{1 / 5-9 \delta} .
$$

By the definition of $T(k, \delta)$ the relation $m \in T(k, \delta)$ means that there exists the pair $q^{\prime}, q^{\prime \prime}$, where $q^{\prime}, q^{\prime \prime} \in \Gamma\left(R_{0}\right), q^{\prime} \neq q^{\prime \prime}$, such that

$$
\left|\left(\vec{p}_{m}(t), \vec{p}_{q^{\prime}}(0)\right)\right|<k^{1 / 5-8 \delta}, \quad\left|\left(\vec{p}_{m}(t), \vec{p}_{q^{\prime \prime}}(0)\right)\right|<k^{3 / 5} .
$$

Let $x \equiv \vec{p}_{m}(t)$. From inequalities (6.24) and (6.25), considering that $\left|\Delta \hat{\Lambda}(t)_{j j}\right|<k^{\delta}$, we obtain the estimates:

$$
\left.|| x\right|^{2}-k^{2}\left|<2 k^{1 / 5},\right|\left(x, q^{\prime}\right)\left|<2 k^{1 / 5-8 \delta},\right|\left(x, q^{\prime \prime}\right) \mid<2 k^{3 / 5} .
$$

This means that $x \in \hat{\pi}_{q^{\prime} q^{\prime \prime}}$ (see (6.13)). Hence, $t \in \mathcal{K} \hat{\pi} \equiv \hat{\Upsilon}_{q}(k, V, \delta)_{5}$. However, $\hat{\chi}_{q}(k, V, \delta) \cap \hat{\Upsilon}_{q}(k, V, \delta)_{5}=\emptyset$. This contradiction proves that condition $5^{\prime}$ holds.

Finally, we prove that $j$ is determined uniquely. Suppose, there exists $m \neq j$, such that $\vec{p}_{m}(t) \in \hat{S}_{q}(k,-\delta)$ and conditions $1^{\prime}-5^{\prime}$ are satisfied. Since $\vec{p}_{m}(t) \in \hat{S}_{q}(k,-\delta)$, we see that $m \in \Pi_{q}\left(k^{1 / 5}\right)$. Therefore, estimate (3.11) holds. But, this contradicts the assumption that $\vec{p}_{m}(t)$ and $\vec{p}_{j}(t)$ belong to $\hat{S}_{q}(k,-\delta)\left(p_{j}^{2}(t)+\Delta \hat{\Lambda}(t)_{j j}=p_{m}^{2}(t)+\Delta \hat{\Lambda}(t)_{m m}=k^{2}\right)$. Estimates (6.14)-(6.26) are stable with respect to a perturbation of order $k^{-2-2 \delta}$. Therefore conditions $1^{\prime}-5^{\prime}$ hold in $\left(k^{-2-2 \delta}\right)$-neighbourhood of $\hat{\chi}_{q}^{0}(k, V, \delta), j$ being uniquely determined from the relation $\left|p_{j}^{2}(t)+\Delta \hat{\Lambda}(t)_{j j}-k^{2}\right|<k^{-2-2 \delta}$. The lemma is proved.

Next, we prove that the set $\hat{\chi}_{q}^{0}(k, V, \delta)$ has an asymptotically full measure on the set $\hat{\mu}_{q}(k, \delta)$ (see Lemma 1). To prove this we verify that each of the sets $\hat{\Upsilon}_{q}(k, V, \delta)_{i}, i=1, \ldots, 5$ has an asymptotically full measure on the set $\hat{\mu}_{q}(k, \delta)$. Lemmas $9-14$ are devoted to the proofs of these assertions. Lemma 8 estimates the area of $\hat{S}_{q}(k,-\delta) \cap \hat{\Pi}_{m}(k, \xi)$. Technically complicated, but nevertheless important parts of the proofs, are carried out to Appendixes at the end of the paper. 
Firstly, we introduce some notations. Let $T_{q}$ be the body of the torus with the radii equal to $k$ and $k^{\prime}, k^{\prime} \equiv \sqrt{k^{2}-p_{q}^{2}(0) / 4-\varphi\left(\vec{p}_{q}(0) / 2\right)}$, the main circle $O_{q}$ of the radius $k^{\prime}$ being centered at the point $\vec{p}_{q}(0) / 2$ and lying in the plane orthogonal to $\vec{p}_{q}(0)$. Thus,

$$
\begin{gathered}
T_{q}=\left\{x:\left|x-x_{s}\right| \leq k, x_{s} \in O_{q}\right\}, \\
O_{q}=\left\{x:\left|x-\vec{p}_{q}(0) / 2\right|=k^{\prime},\left(x, \vec{p}_{q}(0)\right)=0\right\} .
\end{gathered}
$$

It is clear that the sphere $\left|x-\vec{p}_{m}(0)\right|=k$, intersects with the circle $O_{q}$ if and only if $\vec{p}_{m}(0)$ belongs to $T_{q}$. Let $\rho_{q m}$ be the distance from $\vec{p}_{m}(0)$ to the torus ( $\rho_{m q}$ is positive if $\vec{p}_{m}(0)$ is inside torus and negative otherwise).

Let us estimate the area of $\hat{S}_{q}(k,-\delta) \cap \hat{\Pi}_{m}(k, \xi)$. to undestand the structure of $\hat{S}_{q}(k,-\delta) \cap \hat{\Pi}_{m}(k, \xi)$ note that in the case $\varphi_{0}(x)=0$ this is the intersection of the shpere $|x|=k$ and two plane layers: $\Pi_{q}(k,-\delta)$ and $\Pi_{m}(k, \xi)$. So, this is a vicinity on the sphere of two points, which are the intersection of two circles. When $\varphi_{0}(x) \neq 0$, the picture is a little bit "curve". Let us give more precise description of these two points. Using the definitions of $\hat{S}_{q}(k,-\delta)$ and $\Pi_{m}(k, \xi)$ it is easy to show that

$$
\begin{gathered}
\hat{S}_{q}(k,-\delta) \cap \hat{\Pi}_{m}(k, \xi)=\hat{S}_{q}(k,-\delta) \cap \hat{S}_{m}(k, \xi)= \\
\left\{x:|x|^{2}+\varphi_{0}(x)=k^{2},\left.|| x\right|^{2}-\left|x+\vec{p}_{q}(0)\right|^{2} \mid<k^{\delta}\right. \\
\left.\left.|| x\right|^{2}+\varphi_{0}(x)-\left|x+\vec{p}_{m}(0)\right|^{2} \mid<k^{-\xi} \cdot\right\}
\end{gathered}
$$

Thus, $\hat{S}_{q}(k,-\delta) \cap \hat{\Pi}_{m}(k, \xi)$ is a neighbourhood on the surface $|x|^{2}+\varphi_{0}(x)=$ $k^{2}$ of the points defined by the equations

$$
\begin{gathered}
|x|^{2}+\varphi_{0}(x)=k^{2}, \\
|x|^{2}-\left|x+\vec{p}_{q}(0)\right|^{2}=0, \\
\left|x+\vec{p}_{m}(0)\right|^{2}=k^{2} .
\end{gathered}
$$

It is easy to see that this equations define the intersection of the circle $O_{q}$ with the sphere $\left|x+\vec{p}_{m}(0)\right|=k$, which is, obviously, two points. As it is noted above $O_{q}$ intersects with the sphere if and only if $\rho_{q m}$ is positive. Moreover, it turns out that the area of $\hat{S}_{q}(k,-\delta) \cap \hat{\Pi}_{m}(k, \xi)$ essentially depends on $\rho_{q m}$. Naturally, it depends on $k, \delta, \xi$. The following lemma give estimates for the area of $\hat{S}_{q}(k,-\delta) \cap \hat{\Pi}_{m}(k, \xi)$. 
Lemma 6.2. If $8 p_{q}(0)<k, 0<\delta<1,-1<\xi<1$, then the following estimates for $s\left(\hat{S}_{q}(k,-\delta) \cap \hat{\Pi}_{m}(k, \xi)\right)$ hold:

$$
s\left(\hat{S}_{q}(k,-\delta) \cap \hat{\Pi}_{m}(k, \xi)\right) \leq c k^{\delta-\xi} p_{q}^{-1}(0) p_{m}(0)_{\perp}^{-1 / 2} \rho_{m q}^{-1 / 2},
$$

when $\rho_{m q}>k^{-2 \delta}$;

$s\left(\hat{S}_{q}(k,-\delta) \cap \hat{\Pi}_{m}(k, \xi)\right) \leq c k^{\delta / 2-\xi+3 / 2} p_{q}^{-1 / 2}(0) p_{m}(0)^{-3 / 2}\left(4 k^{2}-p_{m}^{2}(0)\right)^{-1 / 4}$,

when $\rho_{m q}<k^{\delta} ; k^{3 \delta}<p_{m}(0)<2 k-k^{-1+3 \delta}$ and

$$
s\left(\hat{S}_{q}(k,-\delta) \cap \hat{\Pi}_{m}(k, \xi)\right) \leq c k^{\delta-\xi / 2} p_{q}^{-1}(0),
$$

when $\rho_{m q}<k^{\delta} ; p_{m}(0)>2 k-k^{\delta}$.

The proof is only a slight modification of Lemma 5 in [8], where we consider the area of $\hat{S}_{q}(k,-\delta) \cap \hat{\Pi}_{m}(k, \xi)$ for $\varphi_{0}=0$, i.e., the case when $\hat{S}_{q}(k,-\delta)$ is a plane-spherical layer and $\hat{\Pi}_{m}(k, \xi)$ is a plane layer. We give it in Appendix 1.

Lemma 6.3. For $0<\delta<10^{-3}$ and sufficiently large $k, k>k_{0}(V, \delta)$ the following estimate holds:

$$
\frac{\left.s\left(\hat{\mu}_{q}(k, \delta) \cap \hat{\Upsilon}_{q}(k, V, \delta)_{1}\right)\right)}{s\left(\hat{\mu}_{q}(k, \delta)\right)}<k^{-3 \delta}
$$

Proof. By the definition of $\hat{\Upsilon}_{q}(k, V, \delta)_{1}$ (see (6.4)) we have

$$
s\left(\hat{\Upsilon}_{q}(k, V, \delta)_{1}\right) \leq \sum_{m \in \Omega_{1}} s\left(\hat{S}_{q}(k, \delta) \cap \hat{\Pi}_{m}(k,-1+10 \delta)\right) .
$$

Taking into account that $\Omega_{1}$ contains less than $c k^{3 \delta}$ elements, we obtain:

$$
s\left(\hat{\Upsilon}_{q}(k, V, \delta)_{1}\right) \leq c k^{3 \delta} \max _{m \in \Omega_{1}} s\left(\hat{S}_{q}(k,-\delta) \cap \hat{\Pi}_{m}(k,-1+10 \delta)\right) .
$$

We prove now that

$$
2 \rho_{m q}>k^{-\delta}
$$


Indeed, by the definition of $\rho_{m q}$, we have

$$
\left(k-\rho_{m q}\right)^{2}=\left(k^{\prime}-r_{m}\right)^{2}+z_{m}^{\prime 2}, \quad z_{m}^{\prime}=z_{m}-p_{q}(0) / 2,
$$

where $r_{m} \equiv p_{m}(0)_{\perp}$ is the absolute value of the projection of the vector $\vec{p}_{m}(0)$ onto the plane orthogonal to $\vec{p}_{q}(0)$ and $z_{m}$ is the projection of $\vec{p}_{m}(0)$ onto $\vec{p}_{q}(0)$. It is clear that $r_{m}=\left(p_{m}^{2}(0)-z_{m}^{2}\right)^{1 / 2}$. Taking into account that $z_{m}^{2}+r_{m}^{2}=p_{m}^{2}(0)=O\left(k^{2 \delta}\right)$ we get

$$
\rho_{m q}\left(2 k-\rho_{m q}\right)=2 k r_{m}+O\left(k^{2 \delta}\right) .
$$

From the relation

$$
r_{m}=\left|\left[\vec{p}_{m}(0), \vec{p}_{q}(0)\right]\right| p_{q}^{-1}(0),
$$

considering that the vectors $\vec{p}_{m}(0)$ and $\vec{p}_{q}(0)$ are linearly independent we obtain $r_{m}>c p_{q}^{-1}(0)>c_{1} k^{-\delta}$. Estimate (6.34) follows from the last inequality and (6.36). Using formula (6.36) and taking into account that $r_{m} \equiv p_{m}(0)_{\perp}$ and $\rho_{m q}>k^{-2 \delta}$, we obtain from (6.28) that

$$
s\left(\hat{S}_{q}(k,-\delta) \cap \hat{\Pi}_{m}(k,-1+10 \delta)\right)<c k^{1-8 \delta} p_{q}(0)^{-1} .
$$

Noting that

$$
s\left(\hat{\mu}_{q}(k, \delta)\right) \approx k^{1+\delta} p_{q}(0)^{-1},
$$

we get

$$
s\left(\hat{S}_{q}(k,-\delta) \cap \Pi_{m}(k,-1+10 \delta)\right) \leq k^{-9 \delta} s\left(\hat{\mu}_{q}(k, \delta)\right) .
$$

Using estimate (6.40) in inequality (6.33) yields (6.31). The lemma is proved.

Lemma 6.4. For $0<\delta<10^{-3}$ and sufficiently large $k, k>k_{0}(V, \delta)$, the following estimate holds:

$$
\frac{\left.s\left(\hat{\mu}_{q}(k, \delta) \cap \hat{\Upsilon}_{q}(k, V, \delta)_{3}\right)\right)}{s\left(\hat{\mu}_{q}(k, \delta)\right)}<k^{-3 \delta} .
$$

Proof. By the definition of $\hat{\Upsilon}_{q}(k, V, \delta)_{3}$ we have

$$
\left.s\left(\hat{\mu}_{q}(k, \delta) \cap \hat{\Upsilon}_{q}(k, V, \delta)_{3}\right) \leq s\left(\hat{\Upsilon}_{q}(k, V, \delta)_{3}\right)\right) \leq \sum_{m} s\left(\hat{S}_{q}(k,-\delta) \cap \hat{\Pi}_{m}(k, 1+\delta)\right) .
$$

Arguing further as in Lemma 6 in [8] and using inequalities (6.28)-(6.30) we verify relation (6.41). The lemma is proved. 
Lemma 6.5. For $0<\delta<10^{-3}$ and sufficiently large $k, k>k_{0}(V, \delta)$ the following estimate holds:

$$
\frac{\left.s\left(\hat{\mu}_{q}(k, \delta) \cap \hat{\Upsilon}_{q}(k, V, \delta)_{2}\right)\right)}{s\left(\hat{\mu}_{q}(k, \delta)\right)}<k^{-4 \delta} .
$$

The proof of this lemma is very technical, so in this consideration we describe the principal steps. Technically complicated parts we send to Appendixes.

Using the definition of $\hat{\Upsilon}_{q}(k, V, \delta)_{2}$ (see (6.5)- (6.8)) we obtain

$$
\begin{gathered}
s\left(\hat{\mu}_{q}(k, \delta) \cap \hat{\Upsilon}_{q}(k, V, \delta)_{2}\right) \leq \\
N \max _{n=1, \ldots, N} \sum_{|i|<k^{\delta}} \sum_{m \in Q_{n i}} s\left(\hat{S}_{q}(k,-\delta) \cap \hat{\Pi}_{m}(k, 1-\delta n)\right),
\end{gathered}
$$

where $Q_{n i}$ is the set of the indeces $m$, such that $m \neq n_{0} q, n_{0} \in Z$, and

$$
\hat{S}_{q}(k,-\delta) \cap \hat{\Pi}_{m}(k, 1-\delta n) \cap \hat{\Pi}_{m+i}(k,-1+\delta n+10 \delta) \neq \emptyset .
$$

Let us estimate the terms of the sum (6.44).

Using estimates (6.28)-(6.30), we obtain that

$$
s\left(\hat{S}_{q}(k,-\delta) \cap \hat{\Pi}_{m}(k, 1-\delta n)\right) \leq k^{-1+\delta(n+1)} p_{q}^{-1}(0) \rho_{m q}^{-1 / 2} p_{m}(0)_{\perp}^{-1 / 2},
$$

when $\rho_{m q}>k^{-2 \delta}$;

$$
k^{1 / 2+\delta(n+1 / 2)} p_{q}^{-1 / 2}(0) p_{m}(0)^{-3 / 2}\left(4 k^{2}-p_{m}^{2}(0)\right)^{-1 / 4},
$$

when $\rho_{m q}<k^{2 \delta} ; k^{3 \delta}<p_{m}(0)<2 k-k^{-1+3 \delta}$ and

$$
s\left(\hat{S}_{q}(k,-\delta) \cap \hat{\Pi}_{m}(k, 1-\delta n)\right) \leq k^{-1 / 2+\delta(n / 2+1)} p_{q}^{-1}(0),
$$

when $\rho_{m q}<k^{\delta} ; p_{m}(0)>2 k-k^{\delta}$.

We represent $Q_{n i}$ in the form $Q_{n i}=\cup_{k=1}^{7} Q_{n i}^{k}$,

$$
Q_{n i}^{1}=\left\{m: m \in Q_{n i}, \rho_{m q}>k^{2 \delta}\right\},
$$


(6.50)

$$
Q_{n i}^{2}=\left\{m: m \in Q_{n i}, \rho_{m q}<k^{2 \delta}, r_{m}>k^{1-6 \delta}, 2 k-r_{m}>k^{1-3 \delta}\right\},
$$

$$
Q_{n i}^{3}=\left\{m: m \in Q_{n i}, \rho_{m q}<k^{2 \delta}, r_{m} \leq k^{1-6 \delta}, p_{m}(0)>k^{2 \delta}\right\},
$$

$$
Q_{n i}^{4}=\left\{m: m \in Q_{n i}, \rho_{m q}<k^{2 \delta}, 2 k-r_{m}<k^{1-3 \delta}, 2 k-p_{m}(0)>k^{3 \delta}\right\},
$$

$$
Q_{n i}^{5}=\left\{m: m \in Q_{n i}, \rho_{m q}<k^{2 \delta}, 2 k-p_{m}(0)<k^{3 \delta},\left|z_{m}\right| \geq k^{2 \delta}\right\},
$$

$$
\begin{gathered}
Q_{n i}^{6}=\left\{m: m \in Q_{n i}, \rho_{m q}<k^{2 \delta}, 2 k-p_{m}(0)<k^{2 \delta},\left|z_{m}\right|<k^{2 \delta}\right\}, \\
Q_{n i}^{7}=\left\{m: m \in Q_{n i}, \rho_{m q}<k^{2 \delta}, p_{m}(0)<k^{2 \delta}\right\}
\end{gathered}
$$

where $r_{m}$ as before is the projection of $\vec{p}_{m}(0)$ onto the plane orthogonal to $\vec{p}_{q}(0)$ and $z_{m}$ is the projection of $\vec{p}_{m}(0)$ onto $\left.\vec{p}_{q} 0\right)$. We break the sum over $Q_{n i}$ in (6.44) into six sums corresponding to $Q_{n i}^{k}, k=1, \ldots, 6$. Taking into account that the summation with respect to $i$ contains less than $c k^{3 \delta}$ terms we get:

$$
s\left(\hat{\mu}_{q}(k, \delta) \cap \hat{\Upsilon}_{q}(k, V, \delta)_{2}\right) \leq c k^{3 \delta} \max _{n=1, \ldots, N,|i|<k^{\delta}}\left(\sum_{k=1}^{7} \Sigma_{i n}^{k}\right)
$$

where

$$
\Sigma_{i n}^{k}=\sum_{m \in Q_{n i}^{k}} s\left(\hat{S}_{q}(k,-\delta) \cap \hat{\Pi}_{m}(k, 1-\delta n)\right)
$$

We estimate each sum $\Sigma_{i n}^{k}$ separately.

1) The estimate of $\Sigma_{i n}^{1}$.

Using inequality (6.46), we obtain

$$
\Sigma_{i n}^{1}<c k^{-1+\delta(n+1)} p_{q}^{-1}(0) \sum_{m \in Q_{n i}^{1}}\left(\rho_{m q} r_{m}\right)^{-1 / 2}
$$


Let us estimate the sum by an integral. Let us define $\rho(y), r(y)$ and $z(y)$ for $y$ just as $\rho_{m q}, r_{m}$ and $z_{m}$ for $\vec{p}_{m}(0)$. Suppose a point $y$ belongs to a cell of the dual lattice, including the point $\vec{p}_{m}(0)$. It is clear that $\left|y-\vec{p}_{m}(0)\right|<A$, $A=2 \pi\left(a_{1}^{-2}+a_{2}^{-2}+a_{3}^{-2}\right)^{1 / 2}$ and $\rho(y)>\rho_{m q}-A$. From the relation $\rho_{m q}>2 k^{\delta}$ it follows that

$$
2 \rho(y)>\rho_{m q}>k^{2 \delta}
$$

Similarly, since

$$
r_{m}>\rho_{m q},
$$

we have $4 r(y)>r_{m}$. Relations (6.59) and (6.60) enable us to estimate the sum $\Sigma_{\text {in }}^{1}$ by the integral $I_{1}$ :

$$
\Sigma_{\text {in }}^{1} \leq c \frac{a_{1} a_{2} a_{3}}{(2 \pi)^{3}} k^{-1+\delta(n+1)} p_{q}^{-1}(0) I_{1},
$$

$$
I_{1}=\int_{\tilde{Q}_{n i}^{1}}(\rho(y) r(y))^{-1 / 2} d y
$$

where $\tilde{Q}_{n i}^{1}$ is the $A$-neighbourhood of $Q_{n i}^{1}$. In Appendix 2 we obtain the formula for $\tilde{Q}_{n i}^{1}$ :

$$
\begin{array}{r}
\tilde{Q}_{n i}^{1} \subset\left\{y: 2 \rho(y)>k^{2 \delta}, \exists x_{0} \in S_{0}:\left|y-x_{0}\right|=k,\right. \\
\left.\left|\left(y-x_{0}, \vec{p}_{i}(0)\right)\right|<4 k^{1-\delta n-10 \delta}+A k(r(y) \rho(y))^{-1 / 2}\right\},
\end{array}
$$

where $S_{0}$ the middle circle of the layer $\hat{S}_{q}(k,-\delta)$ :

$$
S_{0}=\left\{x, x \in \hat{S}_{q}(k,-\delta),|x|^{2}-\left|x+\vec{p}_{q}(0)\right|^{2}=0\right\} .
$$

In Appendix 3 we prove the estimate

$$
I_{1}<c k^{2-n \delta-9 \delta}
$$

Using estimates (6.61) and (6.65) we obtain

$$
\Sigma_{\text {in }}^{1}<c k^{1-4 \delta} p_{q}^{-1}(0) \text {. }
$$

2) The estimate of the sum $\Sigma_{i n}^{2}$. For $m \in Q_{n i}^{2}$ (see (6.50)), estimate (6.47) holds. Note that

$$
p_{m}(0)=\sqrt{r_{m}^{2}+z_{m}^{2}} \geq r_{m}
$$


We estimate $4 k^{2}-p_{m}^{2}(0)$. It follows from relation (6.35) that

$$
4 k^{2}-p_{m}^{2}(0)=2 k\left(2 k-r_{m} k^{\prime} / k\right)+2 \rho_{m q} k-\rho_{m q}^{2}+O\left(k^{1+\delta}\right)>k\left(2 k-r_{m}\right) .
$$

Since $m \in Q_{n i}^{2}$, we have $2 k-r_{m}>k^{1-6 \delta}, r_{m}>k^{1-6 \delta}$. T herefore,

$$
\begin{gathered}
p_{m}(0)>k^{1-6 \delta}, \\
4 k^{2}-p_{m}^{2}(0)>k^{2-6 \delta} .
\end{gathered}
$$

Using the last pair of the estimates in inequality (6.47), we obtain

$$
s\left(\hat{S}_{q}(k,-\delta) \cap \hat{\Pi}_{m}(k, V, 1-\delta n)\right) \leq c p_{q}^{-1}(0) k^{-3 / 2+\delta(n+12)} .
$$

Using this estimate in formula (6.57) for $\Sigma_{i n}^{2}$, we get:

$$
\Sigma_{\text {in }}^{2} \leq c p_{q}^{-1}(0) k^{-3 / 2+\delta(n+12)} \sum_{m \in Q_{n i}^{2}} 1 .
$$

The series on the right of estimate (6.72) can be estimated by the volume of the $A$-neighbourhood $\tilde{Q}_{n i}^{2}$ of the set $Q_{i n}^{2}$ :

$$
\Sigma_{i n}^{2} \leq c p_{q}^{-1}(0) k^{-3 / 2+\delta(n+12)} V\left(\tilde{Q}_{n i}^{2}\right) .
$$

In Appendix 4 we obtain the formula for $\tilde{Q}_{n i}^{k}, k \geq 2$ in a cylindrical coordinates. In Appendix 5 we show that

$$
V\left(\tilde{Q}_{n i}^{2}\right)<k^{1+3 \delta}\left(k^{1-n \delta-10 \delta}+k^{1 / 2+3 \delta}\right) .
$$

It follows from estimates (6.73) and (6.74) that

$$
\Sigma_{i n}^{2} \leq c k^{1-4 \delta} p_{q}^{-1}(0) .
$$

3) The estimate of the sum $\Sigma_{i n}^{3}$. that

For $m \in Q_{n i}^{3}$ the estimate (6.47) is valid. It follows from relation (6.35)

$$
r_{m}^{2}+z_{m}^{2}=2 r_{m} k^{\prime}-2 \rho_{m q} k+\rho_{m q}^{2}+O\left(k^{1+\delta}\right) .
$$

Taking into account that $\rho_{m q}<k^{2 \delta}$, we obtain

$$
p_{m}^{2}(0)=r_{m}^{2}+z_{m}^{2}=2 k^{\prime} r_{m}+O\left(k^{1+\delta}\right) .
$$


Using this inequality and considering that $r_{m}<k^{1-6 \delta}$, we arrive at the relation $4 k^{2}-p_{m}^{2}(0)>k^{2}$. From the last estimate and inequality (6.47), we get:

$$
s\left(\hat{S}_{q}(k,-\delta) \cap \hat{\Pi}_{m}(k, 1-\delta n)\right)<c k^{\delta(n+1 / 2)} p_{q}^{-1}(0) p_{m}^{-3 / 2}(0) .
$$

Substituting (6.78) in formula (6.57) for $\Sigma_{i n}^{3}$, we obtain:

$$
\Sigma_{i n}^{3} \leq c k^{\delta(n+1 / 2)} p_{q}^{-1}(0) \sum_{m \in Q_{n i}^{3}} p_{m}(0)^{-3 / 2} .
$$

Since $p_{m}(0)>k^{2 \delta}$, when $m \in Q_{n i}^{3}$, the series on the right of (6.79) can be estimated by the integral:

$$
\begin{gathered}
\Sigma_{i n}^{3} \leq c k^{\delta(n+1 / 2)} p_{q}^{-1}(0) I_{3}, \\
I_{3}=\int_{\tilde{Q}_{n i}^{3}}\left(r^{2}+z^{2}\right)^{-3 / 4} r d r d z d \vartheta .
\end{gathered}
$$

In Appendix 6 we show that

$$
I_{3} \leq 3 k^{1 / 2-n \delta-9 \delta}+4 k^{2 \delta} .
$$

Substituting the last estimate in the inequality (6.80) yields:

$$
\Sigma_{\text {in }}^{3}<c p_{q}^{-1}(0) k^{1-4 \delta} .
$$

Next, we consider $\Sigma_{i n}^{4}$. Since $m \in Q_{n i}^{4}$, then estimate (6.47) holds. Using relations $p_{m}(0)>r_{m}>k$ and $p_{q}(0)<k^{\delta}$, we rewrite (6.47) in the form:

$$
s\left(\hat{S}_{q}(k,-\delta) \cap \hat{\Pi}_{m}(k, 1-\delta n)<c p_{q}^{-1}(0) k^{-1+\delta(n+1)}\left(4 k^{2}-r_{m}^{2}-z_{m}^{2}\right)^{-1 / 4} .\right.
$$

Substituting estimate (6.84) in formula (6.57) for $\Sigma_{i n}^{4}$, we obtain:

$$
\Sigma_{i n}^{4} \leq c p_{q}^{-1}(0) k^{-1+\delta(n+1)} \sum_{m \in Q_{n i}^{4}}\left(4 k^{2}-r_{m}^{2}-z_{m}^{2}\right)^{-1 / 4} .
$$

It is clear that $\tilde{Q}_{n i}^{4}$ can be described by the formula similar to that for $Q_{n i}^{4}$. Since $4 k^{2}-r_{m}^{2}-z_{m}^{2}>k^{1+2 \delta}$, when $m \in Q_{n i}^{4}$, the series on the right of (6.85) can be estimated by the integral

$$
\Sigma_{i n}^{4} \leq c p_{q}^{-1}(0) k^{-1+\delta(n+1)} I_{4}
$$




$$
I_{4}=\int_{\tilde{Q}_{n i}^{4}} \frac{r d r d z d \vartheta}{\left(4 k^{2}-r^{2}-z^{2}\right)^{1 / 4}} .
$$

In the Appendix 7 we prove that

$$
I_{4} k^{7 / 4-\delta n-6 \delta}+k^{1+2 \delta} .
$$

Using this estimate we (6.86), we get

$$
\Sigma_{\text {in }}^{4} \leq c p_{q}^{-1}(0) k^{1-4 \delta}
$$

for any $\vec{p}_{i}(0), p_{i}(0)<k^{\delta}$.

5) Let us consider $\Sigma_{i n}^{5}$. We prove that $4 k^{2}-p_{m}^{2}(0)>k^{-1+3 \delta}$. Indeed, using (6.35), we obtain

$$
\left(2 k^{\prime}-r_{m}\right) r_{m}=z_{m}^{\prime 2}+2 \rho_{m q} k-\rho_{m q}^{2}+O\left(k^{2 \delta}\right) .
$$

Therefore,

$$
4 k^{2}-r_{m}^{2}-z_{m}^{2}=
$$

$$
\left(z_{m}^{\prime 2}+2 \rho_{m q} k-\rho_{m q}^{2}+O\left(k^{2 \delta}\right)\right)\left(2 k+r_{m}\right) r_{m}^{-1}-z_{m}^{2}>z_{m}^{2}>k^{4 \delta} .
$$

From this $2 k-p_{m}(0)>k^{-1+3 \delta}$. Thus, estimate (6.47) holds. Using relations $p_{m}(0)>r_{m}>k, p_{q}(0)<k^{\delta}$, we rewrite (6.47) in the form (6.84). Taking into account (6.91), we obtain

$$
\Sigma_{i n}^{5} \leq k^{-1+\delta(n+1)} p_{q}^{-1}(0) \sum_{Q_{n i}^{5}} 1 .
$$

Obviously,

$$
\Sigma_{i n}^{5} \leq k^{-1+\delta(n+1)} p_{q}^{-1}(0) V\left(\tilde{Q}_{n i}^{5}\right),
$$

where $\tilde{Q}_{n i}^{5}$ is the $A$-neighbourhood of $Q_{n i}^{5}$. In Appendix 8 we show that

$$
V\left(\tilde{Q}_{n i}^{5}<k^{7 / 4-\delta n-6 \delta}+k^{1+2 \delta} .\right.
$$

Using this estimate we get

$$
\Sigma_{i n}^{5} \leq c p_{q}^{-1}(0) k^{1-4 \delta}
$$

for any $\vec{p}_{i}(0), p_{i}(0)<k^{\delta}$. 
6) Next, we consider $\Sigma_{i n}^{6}$. Using estimate (6.48), we get:

$$
\Sigma_{i n}^{6} \leq k^{-1 / 2+n \delta / 2+\delta} p_{q}^{-1}(0) \sum_{Q_{n i}^{6}} 1 .
$$

It is clear that the sum on the right can be estimated by the volume of the $A$-neighbourhood of $Q_{n i}^{6}$ :

$$
\Sigma_{i n}^{6} \leq k^{-1 / 2+n \delta / 2+\delta} V\left(\tilde{Q}_{n i}^{6}\right) .
$$

It is not hard to show that $\tilde{Q}_{n i}^{6}$ can be described by the formula similar to the formula for $Q_{n i}^{6}$ :

$$
\tilde{Q}_{n i}^{6}=\left\{y:\left|4 k^{2}-r^{2}-z^{2}\right|<2 k^{1+2 \delta}, \rho<2 k^{\delta},|z|<k^{2 \delta}\right\} .
$$

It is not hard to show that $\tilde{Q}_{n i}^{6}$ belongs to the $\left(2 k^{2 \delta}\right)$-neighbourhood of the circle: $r=2 k, z=0$. Therefore, $V\left(\tilde{Q}_{n i}^{6}\right)<c k^{1+2 \delta}$. Using the last estimate in the inequality (6.96), we get:

$$
\Sigma_{\text {in }}^{6}<c p_{q}^{-1}(0) k^{1-4 \delta} .
$$

7) Finally, we consider $\Sigma_{i n}^{7}$. We proved in Lemma 5 that $\rho_{m q}>k^{-2 \delta}$ (see (6.34)), when $r_{m}^{2}+z_{m}^{2}<k^{2 \delta}$. Arguing as in the proof of Lemma 5 and using estimate (6.46), we obtain the inequality:

$$
\Sigma_{\text {in }}^{7}<c p_{q}^{-1}(0) k^{1-4 \delta} .
$$

It follows from (6.56) and estimates (6.66), (6.75), (6.83), (6.89), (6.95), (6.97) and (6.98) that

$$
s\left(\hat{\mu}_{q}(k, \delta) \cap \hat{\Upsilon}_{q}(k, V, \delta)_{2}\right)<c p_{q}^{-1}(0) k^{1-4 \delta} .
$$

Taking into account (6.39), we get (6.43). The lemma is proved.

Lemma 6.6. For $0<\delta<10^{-2}$ and sufficiently large $k, k>k_{0}(V, \delta)$, the following estimate holds:

$$
\frac{s\left(\hat{\mu}_{q}(k, \delta) \cap \hat{\Upsilon}(k, V, \delta)_{4}\right)}{s\left(\hat{\mu}_{q}(k, \delta)\right)}<c k^{-2 \delta}, c \neq c(k, \delta) .
$$

Proof. Using formula (6.10) for $\hat{\Upsilon}(k, V, \delta)_{4}$ and the inequality $\left|q^{\prime}\right|<k^{\delta}$ we obtain

$$
s\left(\hat{\Upsilon}(k, V, \delta)_{4} \cap \hat{\mu}_{q}(k, \delta)\right) \leq c k^{3 \delta} \max _{q^{\prime} \in \Gamma\left(R_{0}\right)} s\left(\hat{\Upsilon}_{q}(k, V, \delta)_{4 q^{\prime}} \cap \hat{\mu}_{q}(k, \delta)\right) .
$$


We prove that

$$
\frac{s\left(\hat{\mu}_{q}(k, \delta) \cap \hat{\Upsilon}_{q}(k, V, \delta)_{4 q^{\prime}}\right)}{s\left(\hat{\mu}_{q}(k, \delta)\right)}<k^{-5 \delta}
$$

Then, estimate (6.99) easily follows from inequalities (6.100) and (6.101).

In the case of linearly independent $q$ and $q^{\prime}$ the proof of estimate (6.101) is similar to that of Lemma 3 in [1]. Indeed, the layer $\hat{S}_{q}(k,-\delta)$ consists of the pieces $S_{k j} \cap \hat{S}_{q}(k,-\delta)$, where

$$
S_{k j}=\left\{t, t \in K, p_{j}^{2}(t)+\hat{\Delta} \Lambda_{j j}(t)=k^{2}\right\}
$$

Using estimate (6.28), it is not hard to show that the sum area of the pieces $S_{k j}$, such that $j \in \Pi_{q^{\prime}}\left(k^{1-12 \delta}\right), q^{\prime} \neq q,\left|q^{\prime}\right|<k^{\delta}$, is infinitely small with respect to $s\left(\hat{S}_{q}(k,-\delta)\right)$, namely

$$
s\left(\cup_{j \in \Pi_{q^{\prime}}\left(k^{1-12 \delta}\right)} S_{k j}\right)<c k^{-6 \delta} s\left(\hat{S}_{q}(k,-\delta)\right) .
$$

Considering relation (6.103), we obtain:

$$
\begin{gathered}
s\left(\hat{\mu}_{q}(k, \delta) \cap \hat{\Upsilon}_{q}(k, V, \delta)_{4 q^{\prime}}\right)< \\
\sum_{j \notin \Pi_{q}\left(k^{1-12 \delta}\right)} s\left(S_{k j} \cap \hat{\Upsilon}_{q}(k, V, \delta)_{4 q^{\prime}}\right)+O\left(k^{-6 \delta}\right) s\left(\hat{S}_{q}(k,-\delta)\right)
\end{gathered}
$$

$$
<c s\left(\hat{S}_{q}(k,-\delta)\right)\left(\sup _{j \notin \Pi_{q}\left(k^{1-12 \delta}\right)} s\left(S_{k j} \cap \hat{\Upsilon}_{q}(k, V, \delta)_{4 q^{\prime}}\right)+O\left(k^{-6 \delta}\right)\right) .
$$

To prove (6.101) it suffices to show that

$$
s\left(S_{k j} \cap \hat{\Upsilon}_{q}(k, V, \delta)_{4 q^{\prime}}\right)<k^{-5 \delta}
$$

The proof of this relation is quite similar to that of estimate (4.4) in [1], where instead of the inequality $\left|\nabla \Lambda_{m m}(t)\right|<k^{\eta}$ we use the following one: $\left|\nabla \hat{\Lambda}_{m m}(t)-\nabla \hat{\Lambda}_{j j}(t)\right|<k^{\eta}, \quad \eta=1 / 5$. Thus,

$$
s\left(\hat{\mu}_{q}(k, \delta) \cap \hat{\Upsilon}_{q}(k, V, \delta)_{4 q^{\prime}}\right)<c k^{1-4 \delta}
$$

when $q^{\prime} \neq \alpha q, \alpha \in R$. Next, suppose $q^{\prime}=\alpha q, \alpha \in R$. It is clear that

$$
\hat{\Upsilon}_{q}(k, V, \delta)_{4 q^{\prime}}=\hat{\Upsilon}_{q}(k, V, \delta)_{4 q^{\prime}}^{\prime} \cap \hat{\Upsilon}_{q}(k, V, \delta)_{4 q^{\prime}}^{\prime \prime}
$$


where

$$
\hat{\Upsilon}_{q}(k, V, \delta)_{4 q^{\prime}}^{\prime}=\left\{t:\left|p_{m}^{2}(t)+\Delta \hat{\Lambda}_{m m}^{q}(t)-p_{j}^{2}(t)-\Delta \hat{\Lambda}_{j j}(t)\right|<k^{-1 / 5-8 \delta},\right.
$$

$$
\left.m \in \Pi_{q}\left(k^{1 / 5}\right), \vec{p}_{m}(0)-\vec{p}_{j}(0)=n \vec{p}_{q}(0), n \in Z\right\}
$$

Naturally,

$$
\hat{\Upsilon}_{q}(k, V, \delta)_{4 q^{\prime}}^{\prime \prime}=\left\{t:\left|p_{m}^{2}(t)+\Delta \hat{\Lambda}_{m m}^{q}(t)-p_{j}^{2}(t)-\Delta \hat{\Lambda}_{j j}(t)\right|<k^{-1 / 5-8 \delta},\right.
$$

$$
\left.m \in \Pi_{q}\left(k^{1 / 5}\right), \vec{p}_{m}(0)-\vec{p}_{j}(0) \neq n \vec{p}_{q}(0), n \in Z\right\} .
$$

We consider $\hat{\Upsilon}_{q}(k, V, \delta)_{4 q^{\prime}}^{\prime}$. Arguing as in [1], we obtain:

$$
p_{m}^{2}(t)+\Delta \hat{\Lambda}_{m m}^{q}(t)-p_{j}^{2}(t)-\Delta \hat{\Lambda}_{j j}(t)=\left(\lambda_{l(m)}-\lambda_{l(j)}\right)(\tau(t))+O\left(k^{-k^{2 / 5}}\right),
$$

where $\lambda_{l}(\tau)$ are eigenvalues of some one-dimensional periodic Schrödinger equation; $l(m), l(j), \tau(t)$ are determined by the formulae:

$$
\begin{gathered}
l(m)=\left[\left(\vec{p}_{m}(t), \vec{p}_{q}(0)\right) p_{q}^{-2}(0)\right], \quad l(j)=\left[\left(\vec{p}_{j}(t), \vec{p}_{q}(0)\right) p_{q}^{-2}(0)\right], \\
\tau(t)=2 \pi\left(\vec{p}_{m}(t), \vec{p}_{q}(0)\right) p_{q}^{-2}(0)-2 \pi l(m) . .
\end{gathered}
$$

It is well-known that for eigenvalues of a one-dimensional Schrödinger equation with a smooth potential the relation

$$
\left|\left(\lambda_{l(m)}-\lambda_{l(j)}\right)(\tau)\right|<k^{-1 / 5-8 \delta}
$$

can be satisfied only when $\min \left\{\tau^{2},(\tau-\pi)^{2},(\tau-2 \pi)^{2}\right\}<c k^{-1 / 5-8 \delta}$. Therefore

$$
\hat{\Upsilon}_{q}(k, V, \delta)_{4 q^{\prime}}^{\prime} \subset \tilde{M}
$$

$$
\tilde{M}=\left\{x, x \in \hat{S}_{q}(k,-\delta), 2 \pi\left|\left(x, \vec{p}_{q}(0)\right) p_{q}(0)^{-2}-l / 2\right|<k^{-1 / 5-8 \delta}, l \in Z\right\} .
$$

It is not hard to show that $\tilde{M}$ is the union of the layers with the width $\pi^{-1} p_{q}(0) k^{-1 / 5-8 \delta}$ that that all of them belong to $\hat{S}_{q}(k,-\delta)$ and, so there are no more than $k^{\delta}$ of them, we obtain the estimate

$$
s\left(\hat{S}_{q}(k,-\delta) \cap \tilde{M}\right)<c k^{4 / 5-7 \delta} .
$$


Using relations (6.111), (6.112), we obtain:

$$
s\left(\hat{\Upsilon}_{q}(k, V, \delta)_{4 q^{\prime}}^{\prime}\right)<c p_{q}^{-1}(0) k^{1-4 \delta},
$$

It remains to consider $\hat{\Upsilon}_{q}(k, V, \delta)_{4 q^{\prime}}^{\prime \prime}$. It is clear that

$$
\hat{\Upsilon}_{q}(k, V, \delta)_{4 q^{\prime}}^{\prime \prime} \subset \cup_{m \in Q}\left(\hat{S}_{q}(k,-\delta) \cap \hat{\Pi}_{m}^{\prime}(k, 1 / 5+8 \delta)\right)
$$

where

$$
\begin{gathered}
\hat{\Pi}_{m}^{\prime}(k, 1 / 5+8 \delta)= \\
\left\{x:\left.|| x\right|^{2}+\varphi(x)-\left|x+\vec{p}_{m}(0)\right|^{2}-\varphi\left(x+\vec{p}_{m}(0)\right) \mid<k^{-1 / 5-8 \delta}\right\} . \\
Q=\left\{m:\left|\left(\vec{p}_{m}(0), \vec{p}_{q}(0)\right)\right|<k^{1 / 5}\right\} .
\end{gathered}
$$

It is not hard to show that the set $\hat{S}_{q}(k,-\delta) \cap \hat{\Pi}_{m}^{\prime}(k, 1 / 5+8 \delta)$ satisfies the estimates similar to (6.28), (6.30) and inequality $(6.29)$ when $p_{m}(0)>$ $k^{1 / 5+\delta}$. Further considerations will be quite similar to those in Lemma 11. Indeed, we estimate $s\left(\hat{\Upsilon}_{q}(k, V, \delta)_{4 q^{\prime}}^{\prime \prime}\right)$ as follows:

$$
s\left(\hat{\Upsilon}_{q}(k, V, \delta)_{4 q^{\prime}}^{\prime \prime}\right) \leq \sum_{k=1}^{6} \Sigma^{k},
$$

where

$$
\Sigma^{k}=\sum_{m \in Q^{k}} s\left(\hat{S}_{q}(k,-\delta) \cap \hat{\Pi}_{m}^{\prime}(k, 1 / 5+8 \delta)\right)
$$

the sets $Q^{k}$ being given by the formula

$$
Q^{1}=\left\{m: m \in Q, \rho_{m q}>k^{2 \delta}\right\},
$$

$$
Q^{2}=\left\{m: m \in Q, \rho_{m q}<k^{2 \delta}, r_{m}>k^{1-\delta}, 2 k-r_{m}>k^{1-6 \delta}\right\},
$$

$$
Q^{3}=\left\{m: m \in Q, \rho_{m q}<k^{2 \delta}, r_{m} \leq k^{1-\delta}, p_{m}(0)>k^{2 \delta}\right\}
$$


(6.122)

$$
Q^{4}=\left\{m: m \in Q, \rho_{m q}<k^{2 \delta}, 2 k-r_{m}<k^{1-6 \delta}, 2 k-p_{m}(0)>k^{2 \delta}\right\},
$$

$$
Q^{5}=\left\{m: m \in Q, \rho_{m q}<k^{2 \delta}, 2 k-p_{m}(0)<k^{2 \delta},|z| \geq k^{2 \delta}\right\},
$$

$$
Q^{6}=\left\{m: m \in Q, \rho_{m q}<k^{2 \delta}, 2 k-p_{m}(0)<k^{2 \delta},|z|<k^{2 \delta}\right\}
$$

$$
Q^{7}=\left\{m: m \in Q, \rho_{m q}<k^{2 \delta}, p_{m}(0)<k^{2 \delta}\right\}
$$

We estimate the sums $\Sigma^{k}$ in Appendix 9. Adding the inequalities for $\Sigma^{k}$ we get

$$
s\left(\hat{\Upsilon}_{q}(k, V, \delta)_{4 q^{\prime}}^{\prime \prime}\right)<c p_{q}^{-1}(0) k^{1-4 \delta}
$$

Adding (6.113) and (6.126), we get

$$
s\left(\hat{\Upsilon}_{q}(k, V, \delta)_{4 q^{\prime}}\right)<c p_{q}^{-1}(0) k^{1-4 \delta} .
$$

Taking into account (6.39) we get (6.99). The lemma is proved.

Suppose $\mu^{\prime}(k, \delta) \subset \hat{\mu}_{q}(k, \delta)$. Let $\Gamma\left(\mu^{\prime}(k, \delta), k^{-r}\right)$ be the $\left(k^{-r}\right)$ neighbourhood in $K$ of the surface $\mu^{\prime}(k, \delta)$.

Lemma 6.7. Suppose $0<\delta<10^{-2}$ and $\mu^{\prime}(k, \delta) \subset \hat{\mu}_{q}(k, \delta)$, and $s\left(\mu^{\prime}(k, \delta)\right)>k^{1-2 \delta}$. Then for sufficiently large $k, k>k_{0}(V, \delta)$ the volume of the $\left(k^{-1-3 \delta}\right)$ - neighbourhood of $\mu^{\prime}(k, \delta)$ is greater than $k^{-5 \delta}$ :

$$
V\left(\Gamma\left(\mu^{\prime}(k, \delta), k^{-1-3 \delta}\right)\right)>k^{-5 \delta} .
$$

Proof. We consider the set

$$
\begin{gathered}
\chi=\mathcal{K}\left(\chi_{0} \cap \hat{S}_{q}(k,-\delta)\right), \\
\chi_{0}=\cup_{m \in \Pi_{q}\left(k^{\delta}\right)} \hat{\Pi}_{m}^{\prime}(k, 2 \delta) .
\end{gathered}
$$

It is obvious that

$$
\Gamma\left(\mu^{\prime}, k^{-1-3 \delta}\right) \supset \Gamma\left(\mu^{\prime} \backslash \chi, k^{-1-3 \delta}\right)
$$


The surface $\hat{\mu}_{q}(k, \delta)$ consists of the pieces $S_{k j}\left(t \in S_{k j}\right.$, if $x \in \hat{S}_{q}(k,-\delta)$, $\left.x=\vec{p}_{j}(t)\right)$. We denote the intersection of the $j$-th piece with $\mu^{\prime}$ by $\mu_{j}^{\prime}$. Thus,

$$
\Gamma\left(\mu^{\prime}, k^{-1-3 \delta}\right)=\cup_{j} \Gamma\left(\mu_{j}^{\prime} \backslash \chi, k^{-1-3 \delta}\right) .
$$

Using the definition of $\chi$ we readily show that

$$
\Gamma\left(\mu_{j_{1}}^{\prime} \backslash \chi, k^{-1-3 \delta}\right) \cap \Gamma\left(\mu_{j_{2}}^{\prime} \backslash \chi, k^{-1-3 \delta}\right)=\emptyset, \quad \text { if } j_{1} \neq j_{2}
$$

From this we obtain:

$$
V\left(\Gamma\left(\mu^{\prime}, k^{1-3 \delta}\right)\right)=\sum_{j} V\left(\Gamma\left(\mu_{j}^{\prime} \backslash \chi, k^{1-3 \delta}\right)\right) .
$$

It is not hard to show that

$$
V\left(\Gamma\left(\mu_{j}^{\prime} \backslash \chi, k^{1-3 \delta}\right)\right)=s\left(\mu_{j}^{\prime} \backslash \chi\right) k^{-1-3 \delta}(1+o(1)) .
$$

Therefore,

$$
V\left(\Gamma\left(\mu^{\prime}, k^{1-3 \delta}\right)\right)>\operatorname{cs}\left(\mu^{\prime} \backslash \chi\right) k^{-1-3 \delta} .
$$

Set $\chi$ coincides with $\hat{\Upsilon}_{q}(k, V, \delta)_{4}$ up to replacement of $\Pi_{q}\left(k^{1 / 5}\right)$ by $\Pi_{q}\left(k^{\delta}\right)$ and $\left(1+\left|\left(\vec{p}_{m}(0), \vec{p}_{q^{\prime}}(0)\right)\right|\right)^{-1}$ by $k^{-2 \delta}$. Arguing as in Lemma 12 , we obtain that

$$
s(\chi)<c p_{q}^{-1}(0) k^{1-4 \delta} .
$$

Taking into account the hypothesis of the lemma on $\mu^{\prime}(k, \delta)$, we get

$$
2 s\left(\mu^{\prime} \backslash \chi\right)>k^{1-2 \delta} .
$$

Using the last inequality in relation (6.135) we obtain estimate (6.128).

The lemma is proved.

Lemma 6.8. For $0<\delta<10^{-2}$ and sufficiently large $k, k>k_{0}(V, \delta)$ the following estimate holds:

$$
\frac{s\left(\hat{\mu}_{q}(k, \delta) \cap \hat{\Upsilon}_{q}(k, \delta)_{5}\right)}{s\left(\hat{\mu}_{q}(k, \delta)\right)}<k^{-3 \delta} .
$$


Proof. Suppose inequality (6.138) does not hold. Then,

$$
s\left(\hat{\mu}_{q}(k, \delta) \cap \hat{\Upsilon}_{q}(k, \delta)_{5}\right)>k^{1-2 \delta} .
$$

By the previous lemma

$$
V\left(\Gamma\left(\hat{\mu}_{q}(k, \delta) \cap \hat{\Upsilon}_{q}(k, \delta)_{5}, k^{-1-3 \delta}\right)\right)>k^{-5 \delta} . .
$$

On the other hand

$$
\hat{\mu}_{q}(k, \delta) \cap \hat{\Upsilon}_{q}(k, \delta)_{5} \subset \hat{\Upsilon}_{q}(k, \delta)_{5}=\mathcal{K} \hat{\pi}
$$

(see (6.13). In [1] we proved that

$$
V\left(\Gamma\left(\mathcal{K} \hat{\pi}, k^{-1-3 \delta}\right)\right)<k^{-6 \delta} .
$$

Thus, inequalities (6.139) and (6.140) are in contradiction. This means that estimate (6.138) holds. The lemma is proved.

Lemma 6.9. For $0<\delta<10^{-2}$ and sufficiently large $k, k>k_{0}(V, \delta)$, the following estimate holds:

$$
\frac{s\left(\hat{\mu}_{q}(k, \delta) \backslash \chi_{q}^{0}(k, V, \delta)\right)}{s\left(\hat{\mu}_{q}(k, \delta)\right)}<k^{-4 \delta} .
$$

Proof. From (6.1) it follows that

$$
s\left(\hat{\mu}_{q}(k, \delta) \cap \chi_{q}^{0}(k, V, \delta)\right) \leq \sum_{r=1}^{5} s\left(\hat{\mu}_{q}(k, \delta) \cap \hat{\Upsilon}_{q}(k, V, \delta)_{i}\right) .
$$

Adding estimates (6.31), (6.41), (6.43), (6.99) and (6.138) for $s\left(\hat{\mu}_{q}(k, \delta) \cap\right.$ $\left.\hat{\Upsilon}_{q}(k, V, \delta)_{i}\right), i=1,2,3,4,5$, we obtain inequality (6.141). The lemma is proved.

Geometric Lemma 1 is the union of Lemma 7 and Lemma 15.

Let us consider the set

$$
\hat{\chi}_{q}^{\prime}(k, V, \delta)=\mu_{q}(k,-\delta) \backslash \cup_{i=1}^{6} \Upsilon_{q}(k, V, \delta)_{i},
$$

where $\Upsilon_{q}(k, V, \delta)_{i}, i=1,2,3,4,5$ coincide with $\hat{\Upsilon}_{q}(k, V, \delta)_{i}$ given by formulae (6.4)-(6.13) up to replacement of $\hat{S}_{q}(k,-\delta)$ for $S_{q}(k,-\delta)$ and

$$
\Upsilon_{6}(k, \delta)=\mathcal{K}\left(\cup_{m \in Z^{3}} \Pi_{m}(k, 1+\delta) \cap S_{q}(k,-\delta)\right) .
$$


Lemma 6.10. If $t$ belongs to $\hat{\chi}_{q}^{\prime}(k, V, \delta)$, then there exists $j$, such that $p_{j}^{2}(t)=k^{2}$ and conditions $1^{\prime}-5^{\prime}$ hold. For $t$ in the $\left(k^{-2-2 \delta}\right)$-neighbourhood of $\hat{\chi}_{q}^{\prime}(k, V, \delta)$ there exists $j$ such that $\left|p_{j}^{2}(t)-k^{2}\right|<k^{-1-2 \delta}$, and conditions $1^{\prime}-5^{\prime}$ hold. The set $\hat{\chi}_{q}^{\prime}(k, V, \delta)$ has an asymptotically full measure on $S_{q}(k,-\delta)$ and the following estimates holds:

$$
\frac{s\left(\hat{\chi}_{q}^{\prime}(k, V, \delta)\right)}{s\left(S_{q}(k,-\delta)\right)}<c k^{-\delta}
$$

Proof. It is easy to see that $j$ is uniquely determined from the relation $\left|p_{j}^{2}(t)-k^{2}\right|<k^{-1-6 \delta}$ for any $t$ in $k^{-2-\delta}$-neighbourhood of $\hat{\chi}_{q}^{\prime}(k, V, \delta)$. Indeed, suppose it is not so. Then, there exists $m \neq j$ such that $\left|p_{m}^{2}(t)-k^{2}\right|<$ $k^{-1-2 \delta}$, i.e., $\left|p_{m}^{2}(t)-p_{j}^{2}(t)\right|<2 k^{-1-2 \delta}$. From the last relation it follows that $t$ is in the $\left(k^{-2-2 \delta}\right)$ - neighbourhood of $\Upsilon_{6}(k, \delta)$. But this contradicts the initial assumption that $t$ is in the neighbourhood of $\hat{\chi}_{q}^{\prime}(k, V, \delta)$. Considering just as in Lemma 3 , we check that conditions $1^{\prime}-5^{\prime}$ are satisfied. To obtain estimate (6.144) one has to repeat the considerations of Lemma 6 in [8] which asserts that $\Upsilon_{6}(k, \delta)$ has an asymptotically full measure on $\mu_{q}(k, \delta)$. The lemma is proved.

\section{Appendixes.}

\subsection{Appendix 1 (The Proof of Lemma 8).}

Firstly we prove estimate (6.28). We split the layer $\hat{S}_{q}(k,-\delta)$ into parallel layers $M_{n}(k, \xi+\delta),|n|<k^{\xi+2 \delta}$ of the width $k^{-\xi-\delta}$. It is clear that

$$
s\left(\hat{S}_{q}(k, \delta) \cap \hat{\Pi}_{m}(k, \xi)\right) \leq k^{\xi+2 \delta} \max _{n} s\left(M_{n}(k, \xi+\delta) \cap \hat{\Pi}_{m}(k, \xi)\right) .
$$

Inequality (6.28) immediately follows from the estimate:

$$
s\left(M_{n}(k, \xi+\delta) \cap \hat{\Pi}_{m}(k, \xi)\right) \leq c k^{-2 \xi-\delta} p_{q}^{-1}(0) p_{m}^{-1 / 2}(0)_{\perp} \rho_{m q}^{-1 / 2} .
$$

We verify the last inequality. For each layer $M_{n}(k, \xi+\delta)$ the relation

$$
\varphi_{0}(x)=\varphi_{n}+O\left(k^{-\xi}\right), \quad \varphi_{n} \neq \varphi_{n}(x), \quad\left|\varphi_{n}\right|<k^{\delta} .
$$

holds. Therefore, from the inequality

$$
\left.|| x\right|^{2}+\varphi_{0}(x)-\left|x+\vec{p}_{m}(0)\right|^{2} \mid<k^{-\xi-\delta}
$$


it follows that

$$
\left.|| x\right|^{2}+\varphi_{n}-\left|x+\vec{p}_{m}(0)\right|^{2} \mid<2 k^{-\xi}, \quad m \in Z^{n} .
$$

This means that

$$
M_{n}(k, \xi+\delta) \cap \hat{\Pi}_{m}(k, \xi) \subset
$$

$$
\left\{x: x \in M_{n}(k, \xi+\delta),\left.|| x\right|^{2}+\varphi_{n}-\left|x+\vec{p}_{m}(0)\right|^{2} \mid<2 k^{-\xi}\right\} .
$$

Now we have the intersection of a plane layer and a "quasi"-spherical shell. Arguing as in the proof of Lemma 5 in [8] and taking into account that $\left|\varphi_{n}\right|<k^{\delta}$, we obtain estimate (7.2), and it gives (6.28). Estimate (6.30) is proved similarly.

To prove inequality (6.29) we direct axis $\tilde{x}_{1}$ along $\vec{p}_{m}(0)$. We consider axis $\tilde{x}_{2}$ to be situated in the plane of the vectors $\vec{p}_{q}(0)$ and $\vec{p}_{m}(0)$. We introduce the spherical coordinates $\tilde{R}, \tilde{\vartheta}, \tilde{\varphi}$ such that $\tilde{x}_{1}=\tilde{R} \cos \tilde{\vartheta}, \tilde{x_{2}}=$ $\tilde{R} \sin \tilde{\vartheta} \cos \tilde{\varphi}, \tilde{x_{3}}=\tilde{R} \sin \tilde{\vartheta} \sin \tilde{\varphi}$. Arguing again as in Lemma 5 in [8] and taking into account that $\left|\varphi_{0}(x)\right|<k^{\delta}$, we get:

$$
\begin{gathered}
\hat{S}_{q}(k,-\delta) \cap \hat{\Pi}_{m}(k, 1+\xi) \subset \\
\left\{x:\left|\cos \tilde{\vartheta}-\cos \tilde{\vartheta}_{1}+\tilde{\Phi}(\tilde{R}, \tilde{\vartheta}, \tilde{\varphi})\right|<\tilde{\varepsilon}_{1},\right. \\
\left|\sin \tilde{\vartheta}\left(\cos \tilde{\varphi}-\cos \tilde{\varphi}_{1}\right)\right| \leq \tilde{\varepsilon}_{2}, \\
\left.\tilde{R}=k+O\left(k^{-1}\right)\right\}
\end{gathered}
$$

where $\cos \tilde{\vartheta}_{1}=p_{m}(0) / 2 \tilde{R}, \cos \tilde{\varphi}_{1}=\left(p_{q}^{2}(0)-2 p_{q}(0)_{\|} k \cos \tilde{\vartheta}\right)\left(2 k p_{q}(0)_{\perp} \sin \tilde{\vartheta}\right)^{-1}$ $\Phi(\tilde{R}, \tilde{\vartheta}, \tilde{\varphi})=(2 \tilde{R})^{-1} p_{m}^{-1}(0) \varphi_{0}(x), \quad \tilde{\varepsilon}_{1}=k^{-\xi-1} p_{m}^{-1}(0) / 2$, and $\tilde{\varepsilon}_{2}=$ $k^{-\delta-1} p_{q}(0)_{\perp}^{-1}$. We will prove that from the inequality

$$
\left|\cos \tilde{\vartheta}-\cos \tilde{\vartheta}_{1}+\tilde{\Phi}(\tilde{R}, \tilde{\vartheta}, \tilde{\varphi})\right|<\tilde{\varepsilon}_{1}
$$

it follows that

$$
\left|\cos \tilde{\vartheta}-\cos \tilde{\vartheta}_{1}\right|<\tilde{\varepsilon}_{1} .
$$

Indeed, it is easy to see that

$$
\left|\frac{\partial \Phi}{\partial \cos \tilde{\vartheta}}\right| \leq\left|p_{m}(0) \sin \tilde{\vartheta}\right|^{-1} k^{\delta}
$$


Suppose that for all $\tilde{\vartheta}$ satisfying inequality (7.8) the following estimate holds:

$$
\tilde{\vartheta}:\left|p_{m}(0) \sin \tilde{\vartheta}\right|>2 k^{\delta} \text {. }
$$

Then (7.9) follows from (7.8). If for some $\tilde{\vartheta}$ we have the opposite inequality $\left|p_{m}(0) \sin \tilde{\vartheta}\right| \leq 2 k^{\delta}$, then $(7.8)$ gives

$$
\cos \tilde{\vartheta}_{1}=1+O\left(p_{m}^{-2}(0) k^{2 \delta}\right) .
$$

On the other hand

$$
\cos \tilde{\vartheta}_{1}=p_{m}(0) / 2 k
$$

by the definition of $\tilde{\vartheta}_{1}$. By hypothesis $p_{m}(0)>k^{3 \delta}, 2 k-p_{m}(0)>k^{-1+3 \delta}$. It is not hard to show, using the last two inequalities that the inequalities (7.12) and (7.13) are in contradiction. Thus, (7.11) is proved, and therefore estimate (7.9) holds. Using inequality (7.8) instead of (7.9) in relation (7.7), we get:

$$
M_{n}(k, \xi+\delta) \cap \hat{\Pi}_{m}(k, \xi) \subset
$$

$\left\{x:\left|\cos \tilde{\vartheta}-\cos \tilde{\vartheta}_{1}\right|<\tilde{\varepsilon}_{1},\left|\sin \tilde{\vartheta}\left(\cos \tilde{\varphi}-\cos \tilde{\varphi}_{1}\right)\right| \tilde{\vartheta}_{1} \leq \tilde{\varepsilon}_{2}, \tilde{R}=k+O\left(k^{-1}\right)\right\}$.

Thus, we arrive to the case of a plane layer. This was considered before in the proof of Lemma 5 in [8]. Thus, we obtain estimate (6.29). The lemma is proved.

\subsection{Appendix 2.}

Considering formulae (6.45) and (6.49) we get: $Q_{n i}^{1} \subset\left\{m: \rho_{m q}>k^{2 \delta}, \exists x \in \hat{S}_{q}(k,-\delta):\left.|| x\right|^{2}-\left|x+\vec{p}_{m}(0)\right|^{2}+\varphi_{0}(x) \mid<k^{-1+\delta n}\right.$,

$$
\left.\left|\left(x+\vec{p}_{m}(0), \vec{p}_{i}(0)\right)\right|<2 k^{1-\delta n-10 \delta}\right\}
$$

Let us prove that

$$
\begin{array}{r}
\tilde{Q}_{n i}^{1} \subset\left\{y: 2 \rho(y)>k^{2 \delta}, \exists x_{0} \in S_{0}:\left|y-x_{0}\right|=k,\right. \\
\left.\left|\left(y-x_{0}, \vec{p}_{i}(0)\right)\right|<4 k^{1-\delta n-10 \delta}+A k(r(y) \rho(y))^{-1 / 2}\right\},
\end{array}
$$

where $S_{0}$ the middle circle of the layer $\hat{S}_{q}(k,-\delta)$ :

$$
S_{0}=\left\{x, x \in \hat{S}_{q}(k,-\delta),|x|^{2}-\left|x+\vec{p}_{q}(0)\right|^{2}=0\right\} .
$$


Indeed, let $y \in \tilde{Q}_{n i}^{1}$. Hence, there exists $\vec{p}_{m}(0):\left|\vec{p}_{m}(0)-y\right|<A, \vec{p}_{m}(0) \in$ $Q_{n i}^{1}$. Estimate (6.59) means that the distance from the point $y$ to the circle $S_{0}$ is less than $k-k^{2 \delta} / 2$. Therefore the sphere of the radius $k$ centered at the point $y$ intersects the circle $S_{0}$ at two points $x_{ \pm}$:

$$
\left|y-x_{ \pm}\right|=k, \quad x_{ \pm} \in S_{0}
$$

It remains to prove that

$$
\left|\left(y-x_{ \pm}, \vec{p}_{i}(0)\right)\right|<4 k^{1-\delta n-10 \delta}+k^{1+2 \delta}(r(y) \rho(y))^{-1 / 2} .
$$

Since $m \in Q_{n i}^{1}$, by the definition of $Q_{n i}^{1}$ there exists $x \in \hat{S}_{q}(k, \delta)$ such that

$$
\left|k^{2}+\varphi_{0}(x)-\right| x+\left.\vec{p}_{m}(0)\right|^{2} \mid<k^{-1+\delta n}
$$

and

$$
\left|\left(x+\vec{p}_{m}(0), \vec{p}_{i}(0)\right)\right|<k^{1-\delta n-10 \delta} .
$$

From estimate (7.20), using the obvious inequalities $\left|\vec{p}_{m}(0)-y\right|<A$ and $\left|\varphi(x)-\varphi\left(x_{0}\right)\right| \leq\|V\|$, we get that the point $x$ is in the $(2 A+\|V\|)$ neighbourhood of the sphere centered at $y$ with radius $k$, i.e., in the spherical shell $\{x:|| y-x|-k|<2 A+2\|V\|\}$. We show (see Appendix 2A) that the intersection of this spherical shell and the quasi-spherical layer $\hat{S}_{q}(k,-\delta)$ lies in the $\left(k^{1+\delta}(r(y) \rho(y))^{-1 / 2}\right)$-neighbourhood of the points $x_{ \pm}$. Thus, $x$ lies in this neighbourhood, i.e.,

$$
\left|x-x_{0}\right|<k^{1+\delta}(r(y) \rho(y))^{-1 / 2},
$$

where $x_{0}$ one of the points $x_{ \pm}$. Considering (7.21), (7.22), we arrive at (7.19) and, therefore (7.17).

\subsection{Appendix 2A.}

- Suppose $x$ is in the intersection of the $2(A+\|V\|)$-neighbourhood of the sphere of radius $k$ centered at the point $y$ and the layer $\hat{S}_{q}(k,-\delta)$. We prove that $x$ is in the $\left(k^{1+\delta}(\rho(y) r(y))^{-1 / 2}\right)$-neighbourhood of the points $x_{ \pm}$ defined by (7.17), (7.18); i.e.,

$$
\left|x-x_{0}\right| \leq k^{1+\delta}(\rho(y) r(y))^{-1 / 2},
$$

where $x_{0}$ is one of the points $x_{ \pm}$. 
We direct axis $x_{1}$ along $\vec{p}_{q}(0)$; axis $x_{2}$ is situated in the plane of the vectors $\vec{p}_{q}(0), y$ and directed so that the projection of $y$ on this axis is positive (the vectors $\vec{p}_{q}(0)$ and $y$ are linearly independent because $\rho(y)>k^{2 \delta}$ ). The axis $x_{3}$ is orthogonal to the axes $x_{1}, x_{2}$. In this coordinate system vector $y$ has the form: $(z, r, 0)$. It is clear that vector $x$ satisfies the relations

$$
\begin{gathered}
x_{1}=a \\
x_{2}^{2}+x_{3}^{2}=k^{2}+\varphi_{0}(a)-a^{2} \\
(z-a)^{2}+\left(r-x_{2}\right)^{2}+x_{3}^{2}=(k-l)^{2}
\end{gathered}
$$

for some $a, l:|a|<k^{\delta},|l|<2(\|V\|+A)$. Note that vector $x_{0}$ satisfies the same relations with $a=p_{q}(0) / 2$ and $l=0$ :

$$
\begin{gathered}
x_{01}=p_{q}(0) / 2, \\
x_{02}^{2}+x_{03}^{2}=k^{\prime 2}, \quad k^{\prime 2}=k^{2}+\varphi_{0}\left(p_{q}(0)\right)-p_{q}(0)^{2} / 4 \\
\left(z-p_{q}(0) / 2\right)^{2}+\left(r-x_{02}\right)^{2}+x_{03}^{2}=k^{2} .
\end{gathered}
$$

We prove that

$$
\left|\frac{\partial x}{\partial a}\right|+\left|\frac{\partial x}{\partial l}\right|<\frac{12 k}{\sqrt{\rho(y) r(y)}} .
$$

Then, inequality (7.23) easily follows from relations (7.24)-(7.26). Let us now prove (7.26). Thus, we calculate $\partial x / \partial l$. By differentiation of equations (7.24) we obtain

$$
\begin{array}{r}
\frac{\partial x_{1}}{\partial l}=0 \\
x_{2} \frac{\partial x_{2}}{\partial l}+x_{3} \frac{\partial x_{3}}{\partial l}=0 \\
\left(x_{2}-r\right) \frac{\partial x_{2}}{\partial l}+x_{3} \frac{\partial x_{3}}{\partial l}=k-l .
\end{array}
$$

We consider this as a linear system with respect to $\partial x_{2} / \partial l, \partial x_{3} / \partial l$. Its determinant is:

$$
\Delta=\left|\left(\begin{array}{ll}
x_{2} & x_{3} \\
x_{2}-r & x_{3}
\end{array}\right)\right|=r x_{3} .
$$

Therefore,

$$
\frac{\partial x_{2}}{\partial l}=-(k-l) r^{-1}
$$




$$
\frac{\partial x_{3}}{\partial l}=x_{2}(k-l) r^{-1} x_{3}^{-1} .
$$

From the relations (7.30) and (7.31), taking into account that $\partial x_{1} / \partial l=0$, we get

$$
\sqrt{\left(\frac{\partial x_{2}}{\partial l}\right)^{2}+\left(\frac{\partial x_{3}}{\partial l}\right)^{2}}=(k-l) r^{-1} x_{3}^{-1} \sqrt{k^{2}-\varphi_{0}(a)-a^{2}} \leq 2 k^{2} r^{-1} x_{3}^{-1} .
$$

Similarly,

$$
\sqrt{\left(\frac{\partial x_{1}}{\partial a}\right)^{2}+\left(\frac{\partial x_{2}}{\partial a}\right)^{2}+\left(\frac{\partial x_{3}}{\partial a}\right)^{2}}<2 k^{2} r^{-1} x_{3}^{-1}
$$

Next, we prove that $r x_{3}>k \rho^{1 / 2} r^{1 / 2}$. From (7.24) we easily obtain

$$
x_{3}^{2}+x_{2}^{2}=R_{1}^{2}
$$

$$
x_{3}^{2}+\left(r-x_{2}\right)^{2}=R_{2}^{2}
$$

where

$$
\begin{gathered}
R_{1}^{2}=k^{2}-\varphi_{0}(a)-a^{2}, \\
R_{2}^{2}=(k-l)^{2}-(z-a)^{2} .
\end{gathered}
$$

Hence,

$$
\left(r-2 x_{2}\right) r=R_{2}^{2}-R_{1}^{2}
$$

i.e.,

$$
-2 x_{2}=\left(\left(R_{2}^{2}-R_{1}^{2}\right)-r^{2}\right) r^{-1} .
$$

From relations (7.34) and (7.37) we get

$$
x_{3}^{2}=R_{1}^{2}-\left(R_{2}^{2}-R_{1}^{2}-r^{2}\right)^{2} / 4 r^{2} .
$$

Thus,

$$
4 x_{3}^{2} r^{2}=4 R_{1}^{2} r^{2}-\left(R_{2}^{2}-R_{1}^{2}-r^{2}\right)^{2}=-r^{4}+2\left(R_{2}^{2}+R_{1}^{2}\right) r^{2}-\left(R_{2}^{2}-R_{1}^{2}\right)^{2} .
$$


Considering the right as a polynomial with respect to $r$, we obtain

$$
4 x_{3}^{2} r^{2}=-\left(r-y_{1+}\right)\left(r-y_{1-}\right)\left(r-y_{2+}\right)\left(r-y_{2-}\right),
$$

where $y_{1 \pm}, y_{2 \pm}$ are the roots of the polynomial

$$
y_{1+}=R_{1}+R_{2}, \quad y_{1-}=-\left(R_{1}+R_{2}\right), \quad y_{2+}=R_{1}-R_{2}, y_{2-}=R_{2}-R_{1} .
$$

We define $\rho_{a}$ so that $k-l-\rho_{a}$ is the distance between the point $y$ and the circle $\left\{x: x_{1}=a, x_{2}^{2}+x_{3}^{2}=R_{1}^{2}\right\}$. Then $\rho_{a}$ satisfies the equation

$$
\left(k-l-\rho_{a}\right)^{2}=\left(R_{1}-r\right)^{2}+(z-a)^{2} .
$$

It is easily follows from the last relation and the formula for $R_{2}$ that $2(k-l) \rho_{a}-\rho_{a}^{2}=(k-l)^{2}-\left(R_{1}-r\right)^{2}-(z-a)^{2}=\left(R_{2}-R_{1}+r\right)\left(R_{2}+R_{1}-r\right)=$

$$
=-\left(r-y_{2+}\right)\left(r-y_{1+}\right) \text {. }
$$

Note that $\left|k-l-\rho_{a}-k+\rho\right|<a+O\left(a k^{-1}\right)$, since the circles $S_{0}$ and $S_{a}$ are separated the distance $a+O\left(a k^{-1}\right)$. Hence, $\left|\rho-\rho_{a}\right|<2 a+l$. From the relation $\rho>k^{\delta}$, it follows that $2 \rho_{a}>\rho$. Considering the inequality $\rho_{a}<k$, we obtain

$$
2(k-l) \rho_{a}-\rho_{a}^{2}>\rho k / 2 .
$$

Relations (7.43) and (7.44) together give:

$$
\rho k<-2\left(r-y_{2+}\right)\left(r-y_{1+}\right) .
$$

Note that

$$
r-y_{1-}=r+R_{1}+R_{2}>R_{1}>k / 2 .
$$

Suppose $z>k^{2 \delta}$. Then, $R_{2}<R_{1}$, because $a<k^{\delta}$. Therefore, $y_{2-}<0$ and, naturally,

$$
r-y_{2-}>r .
$$

If $z \leq k^{2 \delta}$, then $R_{2}-R_{1}=O\left(k^{4 \delta-1}\right)$ and using that $2 r>\rho>k^{2 \delta}$, we obtain $r-y_{2-}>r / 2$. Therefore,

$$
r-y_{2-}>r / 2 \text {. }
$$


Substituting inequalities (7.45), (7.46) and (7.48) in the relation (7.40), we get

$$
4 x_{3}^{2} r^{2}>\rho k^{2} r / 8
$$

Hence,

$$
6 x_{3} r>k \sqrt{\rho r} .
$$

Using the last relation in inequalities (7.32) and (7.33) we obtain (7.26). Thus, estimate (7.23) is proved.

\subsection{Appendix 3.}

. We prove the estimate

$$
I_{1}<c k^{2-n \delta-9 \delta}
$$

Firstly, suppose $\vec{p}_{i}(0)$ and $\vec{p}_{q}(0)$ are linearly independent. We introduce the cylindrical coordinates $z, r, \vartheta$, where $z$ is directed alone $\vec{p}_{q}(0)$, and angle $\vartheta$ is assumed to be zero on the projection of the vector $\vec{p}_{i}(0)$ on the plane $z=0$. In these coordinates

$$
\begin{gathered}
\vec{p}_{i}(0)=\left(\gamma_{2}, \gamma_{1}, 0\right), \\
x_{0}=\left(p_{q}(0) / 2, k^{\prime}, \vartheta_{0}\right), x_{0} \in S_{0},
\end{gathered}
$$

where

$$
k^{\prime}=\sqrt{k^{2}-\varphi_{0}\left(x_{0}\right)-p_{q}^{2}(0) / 4}
$$

$\gamma_{2}$ is the projection of the vector $\vec{p}_{i}(0)$ onto $\vec{p}_{q}(0)$ and $\gamma_{1}=\sqrt{p_{i}^{2}(0)-\gamma_{2}^{2}}$. Note that in the cylindrical coordinates $\tilde{Q}_{n i}^{1}$ is described as follows:

$$
\begin{gathered}
\tilde{Q}_{n i}^{1}=\left\{y=(r, z, \vartheta): \rho(y)>k^{2 \delta}, \exists \vartheta_{0}: r^{2}-2 r k^{\prime} \cos \left(\vartheta-\vartheta_{0}\right)+z^{\prime 2}=b,\right. \\
\left.(7.52) \quad\left|\gamma_{1}\left(r \cos \vartheta-k^{\prime} \cos \vartheta_{0}\right)+\gamma_{2} z^{\prime}\right|<4 k^{1-\delta n}+k^{1+\delta}(r \rho)^{-1 / 2}\right\}, \\
b \equiv k^{2}-k^{\prime 2}=\varphi_{0}\left(x_{0}\right)+p_{q}^{2}(0) / 4 .
\end{gathered}
$$

Taking into account that

$$
(\rho-k)^{2}=\left(r-k^{\prime}\right)^{2}+{z^{\prime}}^{2}
$$

we get that the relation $r^{2}-2 r k^{\prime} \cos \left(\vartheta-\vartheta_{0}\right)+{z^{\prime}}^{2}=b$ can be rewritten in the form:

$$
r\left(1-\cos \left(\vartheta-\vartheta_{0}\right)\right)=\rho\left(\frac{k-\rho / 2}{k^{\prime}}\right) .
$$


Thus,

$$
\tilde{Q}_{n i}^{1}=\left\{y=(r, z, \vartheta): \rho>k^{\delta}, \exists \vartheta_{0}: r\left(1-\cos \left(\vartheta-\vartheta_{0}\right)\right)=\frac{\rho(k-\rho / 2)}{k^{\prime}},\right.
$$

$$
\left.\left|\gamma_{1}\left(r \cos \vartheta-k^{\prime} \cos \vartheta_{0}\right)+\gamma_{2} z^{\prime}\right|<4 k^{1-\delta n-2 \delta}+k^{1+\delta}(r \rho)^{-1 / 2}\right\} .
$$

To calculate $I_{1}$ we change the variables: $r, z, \vartheta \rightarrow \vartheta_{0}, l, \vartheta$

$$
\cos \left(\vartheta-\vartheta_{0}\right)=\frac{r^{2}+{z^{\prime}}^{2}-b}{2 r k^{\prime}}, \quad-\pi / 2 \leq \vartheta-\vartheta_{0}<\pi / 2 .
$$

We describe the set $\tilde{Q}_{n i}^{1}$ in the new coordinates. It follows from formula (7.54) and relations $k^{2 \delta}<\rho(y)<k$ and $r(y)<2 k$ that

$$
2\left(1-\cos \left(\vartheta-\vartheta_{0}\right)\right)>k^{-1+2 \delta} .
$$

Thus,

$$
\tilde{Q}_{n i}^{1} \subset\left\{y=\left(\vartheta_{0}, l, \vartheta\right): 0 \leq \vartheta<2 \pi,\right.
$$

$\left.\left|l-\gamma_{1} k^{\prime} \cos \vartheta_{0}\right|<4 k^{1-\delta n-10 \delta}+k^{1+2 \delta}(r(y) \rho(y))^{-1 / 2}, 2\left(1-\cos \left(\vartheta-\vartheta_{0}\right)\right)>k^{-1+2 \delta}\right\}$.

Thedeterminant corresponding to this change of variables is given by the following calculation:

$$
\begin{gathered}
D(y)=\left|\left(\begin{array}{cc}
\frac{\partial \vartheta_{0}}{\partial r} & \frac{\partial \vartheta_{0}}{\partial z} \\
\frac{\partial l}{\partial r} & \frac{\partial l}{\partial z}
\end{array}\right)\right|=\frac{1}{2 k^{\prime} \sin \left(\vartheta-\vartheta_{0}\right)}\left|\left(\begin{array}{cc}
1-\frac{z^{\prime 2}-b}{r^{2}} & \frac{2 z^{\prime}}{r} \\
\gamma_{1} \cos \vartheta & \gamma_{2}
\end{array}\right)\right|= \\
\frac{1}{k^{\prime} r \sin \left(\vartheta-\vartheta_{0}\right)}\left|z^{\prime} \gamma_{1} \cos \vartheta-\gamma_{2} r+\frac{\gamma_{2}\left(r^{2}+z^{\prime 2}-b\right)}{2 r}\right|= \\
\frac{\left|z^{\prime} \gamma_{1} \cos \vartheta-\gamma_{2} r+\gamma_{2} k^{\prime} \cos \left(\vartheta-\vartheta_{0}\right)\right|}{k^{\prime} r \sin \left(\vartheta-\vartheta_{0}\right)} .
\end{gathered}
$$

Introducing the new coordinate $\eta$ orthogonal to $l$ in the plane of variables $z^{\prime}$ and $r$ :

$$
\eta=z^{\prime} \gamma_{1} \cos \vartheta-\gamma_{2} r
$$


and defining $\hat{a}, \alpha_{1}$ and $\alpha_{2}$ by the formulae: $\hat{a}=\sqrt{\gamma_{1}^{2} \cos ^{2} \vartheta+\gamma_{2}^{2}}, \alpha_{1}=$ $\hat{a}^{-1} \gamma_{1} \cos \vartheta$, and $\alpha_{2}=\hat{a}^{-1} \gamma_{2}$, we obtain

$$
D(y)=\frac{\left|\hat{a}\left(\eta \hat{a}^{-1}+\alpha_{2} k^{\prime} \cos \left(\vartheta-\vartheta_{0}\right)\right)\right|}{k^{\prime} r\left|\sin \left(\vartheta-\vartheta_{0}\right)\right|} .
$$

To estimate $D(y)$ we rewrite (7.57) in the form

$$
\left(r-k^{\prime} \cos \left(\vartheta-\vartheta_{0}\right)\right)^{2}+{z^{\prime 2}}^{2}={k^{\prime}}^{2} \cos ^{2}\left(\vartheta-\vartheta_{0}\right)+b .
$$

Using formulae (7.56) and (7.61) for $l$ and $\eta$, we obtain that

$$
\begin{gathered}
\left(\hat{a}^{-1} l-\alpha_{1} k^{\prime} \cos \left(\vartheta-\vartheta_{0}\right)\right)^{2}+\left(\hat{a}^{-1} \eta+\alpha_{2} k^{\prime} \cos \left(\vartheta-\vartheta_{0}\right)\right)^{2}= \\
k^{\prime 2} \cos ^{2}\left(\vartheta-\vartheta_{0}\right)+b .
\end{gathered}
$$

From this, using the relation (7.59) and taking into account that

$$
\begin{gathered}
l=\gamma_{1} k^{\prime} \cos \vartheta_{0}+\tilde{l}, \\
|\tilde{l}|<2 k^{1-\delta n-10 \delta}+k^{1+\delta}(r \rho)^{-1 / 2},
\end{gathered}
$$

we obtain the estimate for the determinant:

$$
D \geq\left(r \sin \left(\vartheta-\vartheta_{0}\right)\right)^{-1} \sqrt{f\left(\vartheta, \vartheta_{0}, l\right)},
$$

where

$$
f\left(\vartheta, \vartheta_{0}, l\right)=
$$

$$
\hat{a}^{2} \cos ^{2}\left(\vartheta-\vartheta_{0}\right)-\left(\gamma_{1} \cos \vartheta_{0}-\gamma_{1} \cos \vartheta \cos \left(\vartheta-\vartheta_{0}\right)+\tilde{l} / k^{\prime}\right)^{2}+\hat{a}^{2} b k^{\prime-2} .
$$

Using relation (7.66), we get the estimate for the integral:

$$
I_{1} \leq \int_{\tilde{Q}_{r m}^{1}} \frac{r^{2} \sin \left(\vartheta-\vartheta_{0}\right)}{\sqrt{f\left(\vartheta, \vartheta_{0}, l\right) r \rho}} d \vartheta d \vartheta_{0} d l
$$

It follows from relations $(7.54),(6.60)$ and the estimate $\rho>k^{2 \delta}$ that

$$
k^{-1+2 \delta}<r / \rho<2\left(1-\cos \left(\vartheta-\vartheta_{0}\right)\right)^{-1} .
$$


Using this relation and producing the change of the variable $\vartheta_{0} \rightarrow \tau, \tau=$ $\cos \left(\vartheta-\vartheta_{0}\right)$, we get

$$
I_{1} \leq \int_{\tilde{Q}_{r m}^{1}} \frac{r}{\sqrt{f(\vartheta, \tau, l)(1-\tau)}} d \tau d \vartheta d l
$$

Taking into account formula (7.59) for $\tilde{Q}_{r m}^{1}$, it is not hard to show that $I_{1} \leq I_{2}+I_{3}$, where $I_{2}$ and $I_{3}$ are the integrals over $\omega_{2}$ and $\omega_{3}$ given by:

$$
\begin{gathered}
\omega_{2}=\left\{y: 0<\vartheta \leq 2 \pi,\left|l-\gamma_{1} k^{\prime} \cos \vartheta_{0}\right|<8 k^{1-\delta n-10 \delta}, 2(1-\tau)>k^{-1+2 \delta}\right\}, \\
\omega_{3}=\left\{y: 0<\vartheta \leq 2 \pi,\left|l-\gamma_{1} k^{\prime} \cos \vartheta_{0}\right|<k^{1+\delta}(r \rho)^{-1 / 2}, 2(1-\tau)>k^{-1+2 \delta}\right\} .
\end{gathered}
$$

Integrating with respect to $l$ and taking into account (7.68), we obtain

$$
I_{2} \leq k^{2-\delta n-10 \delta} \max _{l} \int_{0}^{1-\tau_{0}} \frac{d \tau}{(1-\tau)^{1 / 2}} \int_{0}^{2 \pi} \frac{d \vartheta}{\sqrt{f(\vartheta, \tau, l)}}
$$

$$
I_{3} \leq k^{1+\delta} \max _{l} \int_{0}^{1-\tau_{0}} \frac{d \tau}{1-\tau} \int_{0}^{2 \pi} \frac{d \vartheta}{\sqrt{f(\vartheta, \tau, l)}}
$$

where $\tau_{0}=k^{-1+2 \delta} / 2$. It is not hard to show that

$$
\begin{gathered}
f(\vartheta, \tau, l)=\gamma_{2}^{2} \tau^{2}+\gamma_{1}^{2} \tau^{2} \cos ^{2} \vartheta-\gamma_{1}^{2}\left(1-\tau^{2}\right) \sin ^{2} \vartheta \\
+2 \gamma_{1} k^{\prime-1} \tilde{l} \sqrt{1-\tau^{2}} \sin \vartheta+\tilde{l}^{2} k^{\prime-2}+k^{\prime-2} b\left(\gamma_{2}^{2}+\gamma_{1}^{2} \cos ^{2} \vartheta\right)= \\
-\left(\mu_{1} \sin ^{2} \vartheta+2 \alpha \sin \vartheta+\beta\right)
\end{gathered}
$$

where

$$
\begin{gathered}
\alpha=-\gamma_{1} k^{\prime-1} \tilde{l} \sqrt{1-\tau^{2}}, \\
\beta=-\left(\gamma_{1}^{2}+\gamma_{2}^{2}\right) \tau^{2}-k^{\prime-2} \tilde{l}^{2}-\left(\gamma_{1}^{2}+\gamma_{2}^{2}\right) k^{\prime-2} b \\
\mu_{1}=\gamma_{1}^{2}\left(1+k^{\prime-2} b\right) .
\end{gathered}
$$

We show in Appendix $3 \mathrm{~A}$ that integral with respect to $\vartheta$ has only logarithmic singularities in some points $\tau_{n}=\tau_{n}(l), l=1,2,3,4$. Therefore,

$$
\max _{l} \int_{0}^{1-\tau_{0}} \frac{d \tau}{\sqrt{1-\tau}} \int_{0}^{2 \pi} \frac{d \vartheta}{\sqrt{f}}<k^{2 \delta}
$$


Hence, $I_{2} \leq k^{2-\delta n-8 \delta}$. Similarly, we prove that $I_{3} \leq k^{1+2 \delta}$. Thus,

$$
I_{1}<2 k^{2-\delta n-8 \delta} \text {, when } \gamma_{1} \neq 0 .
$$

If $\gamma_{1}=0$, then we direct the semiaxis $\vartheta=0$ arbitrary. It is not hard to show $f\left(\vartheta, \vartheta_{0}, l\right)=|\gamma|^{2} \tau^{2}+k^{\prime-2} \tilde{l}^{2}+k^{\prime-2}|\gamma|^{2} b^{2}$. Therefore,

$$
I_{2} \leq k \int_{|l|<k^{1-\delta n-10 \delta}} d l \int_{0}^{1-\tau_{0}} \frac{d \tau}{(1-\tau)^{1 / 2}\left(|\gamma|^{2} \tau^{2}+k^{\prime-2} l^{2}+k^{\prime-2}|\gamma|^{2} b^{2}\right)^{1 / 2}}
$$

$$
\leq k \int_{|l|<k^{1-\delta n-10 \delta}} \ln \frac{l}{k} d l \leq k^{2-\delta n-9 \delta} .
$$

Similarly, $I_{3} \leq k^{1+2 \delta}$. Therefore, $I_{1} \leq k^{2-\delta n-9 \delta}$ for any $\vec{p}_{i}(0), p_{i}(0)<k^{\delta}$. The estimate (7.49) is proved.

\subsection{Appendix 3A.}

We prove that for $\gamma_{1} \neq 0$

$$
\tilde{I} \equiv \int_{0}^{2 \pi} f(\vartheta, \tau, l)^{-1 / 2} d \vartheta=\sum_{i=1}^{4} c_{i} \ln \left(\tau-\tau_{i}(l)\right)+c_{0}, \quad c_{i} \neq c_{i}(k), \quad i=0,1,2,3,4 .
$$

It is clear that

$$
\begin{gathered}
\tilde{I}=-\frac{1}{\sqrt{-\mu_{1}}} \int_{0}^{2 \pi} \frac{d \varphi}{\sqrt{\left(\sin \varphi-b_{1}\right)\left(\sin \varphi-b_{2}\right)}}= \\
-\frac{1}{\sqrt{-\mu_{1}}} \int_{-1}^{1} \frac{d \xi}{\sqrt{\left(1-\xi^{2}\right)\left(\xi-b_{1}\right)\left(\xi-b_{2}\right)}}, \\
b_{i}=b_{i}(l, \tau), i=1,2 .
\end{gathered}
$$

It is easy to see from the linear independence of $\vec{p}_{i}(0)$ and $\vec{p}_{q}(0)$ that $\left|\gamma_{1}\right|>k^{-2 \delta}$. Suppose $|\gamma||\tau|\left|\gamma_{1}\right|^{-1}>1 / 2$. Then, in formulae (7.73)-(7.75)) $\alpha \mu_{1}^{-1}=o(1), \beta \mu_{1}^{-1}=\gamma_{1}^{-2}|\gamma|^{2} \tau^{2}+o(1)$; therefore $b_{1} \approx \gamma_{1}^{-1}|\gamma| \tau>1 / 2$, $b_{2} \approx-\gamma_{1}^{-1}|\gamma| \tau<-1 / 2$. Considering the last two equations, it is not hard to show that

$$
\tilde{I} \leq c \ln \left|1-b_{1}(l, \tau)\right|\left|1+b_{2}(l, \tau)\right| .
$$


We see that $\tilde{I}$ has singularities on the curves $b_{1}(l, \tau)=1$ and $b_{2}(l, \tau)=-1$. It is not hard to show that these equation are satisfied by roots $\tau_{1+}, \tau_{1-}, \tau_{2+}$ and $\tau_{2-}$ of a polynomial of the fourth power and $\tau_{1,2 \pm}^{2}=\gamma_{1}^{2}|\gamma|^{-2}+o(1)$, The function $\left(1-b_{1}(l, \tau)\right)\left(1+b_{2}(l, \tau)\right)$ is infinitely differentiable in these points, because

$$
\begin{gathered}
\left(1-b_{1}(l, \tau)\right)\left(1+b_{2}(l, \tau)\right)=\left(1-\sqrt{\tilde{\alpha}^{2}-\tilde{\beta}}\right)^{2}-\tilde{\alpha}^{2}, \\
\tilde{\alpha}^{2}=-\mu_{1}^{-1} \alpha^{2}=\tilde{l}^{2} k^{\prime-2}\left(1-\tau^{2}\right)=o(1), \\
-\tilde{\beta}=\mu_{1}^{-1} \beta=\gamma_{1}^{-2}|\gamma|^{2} \tau^{2}+o(1) \approx 1 .
\end{gathered}
$$

Taking into account that $\left(1-b_{1}(l, \tau)\right)\left(1+b_{2}(l, \tau)\right)$ is differentiable, we obtain estimate (7.79).

If $\gamma\left|\gamma_{1}\right|^{-1} \tau \leq 1 / 2$, then $\left|1-b_{1}\right|>1 / 4,\left|1+b_{1}\right|>1 / 4$. It follows from relation (7.80) that

$$
\tilde{I} \leq\left|\gamma_{1}\right|^{-2} \ln \left|b_{1}-b_{2}\right|=\ln \left|\alpha^{2}-\beta\right| .
$$

Taking into account that $\alpha^{2}-\beta$ is a quadratic polynomial with respect to $\tau$, it is not hard to prove estimate (7.79).

7.6. Appendix 4 (formulae in the cylindrical coordinates for $Q_{n i}^{k}$ and $\tilde{Q}_{n i}^{k}, k \geq 2$ ).

Suppose $\vec{p}_{i}(0)$ and $\vec{p}_{q}(0)$ are linearly independent. We introduce the cylindrical coordinates $z, r, \vartheta$, where $z$ is directed alone $\vec{p}_{q}(0)$, and angle $\vartheta$ is assumed to be zero on the projection of the vector $\vec{p}_{i}(0)$ on the plane $z=0$. In these coordinates

$$
\begin{gathered}
\vec{p}_{i}(0)=\left(\gamma_{2}, \gamma_{1}, 0\right), \\
x_{0}=\left(p_{q}(0) / 2, k^{\prime}, \vartheta_{0}\right), x_{0} \in S_{0},
\end{gathered}
$$

where $k^{\prime}$ is given by (6.35), $\gamma_{2}$ is the projection of the vector $\vec{p}_{i}(0)$ onto $\vec{p}_{q}(0)$ and $\gamma_{1}=\sqrt{p_{i}^{2}(0)-\gamma_{2}^{2}}$.

We prove that $Q_{n i}^{k}$ and $\tilde{Q}_{n i}^{k}, k=2,3,4,5,6,7$ are described by similar formulae:

$$
Q_{n i}^{k} \subset\left\{m:\left|\sqrt{\left(r_{m}-k^{\prime}\right)^{2}+z_{m}^{\prime 2}}-k\right|<k^{\delta},\right.
$$

$$
\left.\left|\gamma_{1}\left(r_{m}-k\right) \cos \vartheta_{m}+\gamma_{2} \hat{z}_{m}\right|<2 k^{1-\delta n-10 \delta}+k^{1+2 \delta} r^{-1 / 2}\right\}
$$




$$
\tilde{Q}_{n i}^{k} \subset\left\{y=(z, r, \vartheta):\left|\sqrt{\left(r-k^{\prime}\right)^{2}+{z^{\prime}}^{2}}-k\right|<2 k^{2 \delta},\right.
$$

$$
\left.\left|\gamma_{1}(r-k) \cos \vartheta+\gamma_{2} z\right|<2 k^{1-\delta n-10 \delta}+k^{1+\delta} r^{-1 / 2}\right\} .
$$

Indeed, the polar coordinates of a point $x$ in $\hat{S}_{q}(k,-\delta)$ are

$$
\begin{gathered}
x=\left(a, \hat{k}, \vartheta_{0}\right), \hat{k} \equiv \sqrt{k^{2}-a^{2}-\varphi_{0}(a)}, \\
|a|<k^{\delta}, 0 \leq \vartheta_{0}<2 \pi .
\end{gathered}
$$

If $m \in Q_{n i}$, then relation (6.45) holds. This means that for some $x$ in $\hat{S}_{q}(k,-\delta)$

$$
\begin{gathered}
\left(r_{m} \cos \vartheta_{m}-\hat{k} \cos \vartheta_{0}\right)^{2}+\left(r_{m} \sin \vartheta_{m}-\hat{k} \sin \vartheta_{0}\right)^{2}+\left(z_{m}-a\right)^{2} \\
=k^{2}-\varphi_{0}(a)+O\left(k^{-1+\delta n}\right)
\end{gathered}
$$

$$
\left|\gamma_{1}\left(r_{m} \cos \vartheta_{m}-\hat{k} \cos \vartheta_{0}\right)+\gamma_{2}\left(z_{m}-a\right)\right|<k^{1-\delta n-10 \delta},
$$

$r_{m}, z_{m}, \varphi_{m}$ being the polar coordinates of the vector $-\vec{p}_{m}(0)$. If $m \in$ $\cup_{k=2}^{7} Q_{n i}^{k}$, then $\rho_{m q}<k^{2 \delta}$, i.e., (see (7.53))

$$
\left|\sqrt{\left(r_{m}-k^{\prime}\right)^{2}+z_{m}^{\prime 2}}-k\right|<k^{2 \delta} .
$$

Using relations (7.85)-(7.87) and the inequalities $|a|<k^{\delta}, 1-\delta n-10 \delta>\delta$, we easily show that for $k=2,3,4,5,6,7$

$$
\begin{gathered}
Q_{n i}^{k} \subset\left\{m: \exists \vartheta_{0}:\left|\sqrt{\left(r_{m}-k^{\prime}\right)^{2}+z_{m}^{\prime 2}}-k\right|<k^{2 \delta},\right. \\
\left|\gamma_{1}\left(r_{m} \cos \vartheta_{m}-\hat{k} \cos \vartheta_{0}\right)+\gamma_{2} \hat{z}_{m}\right|<2 k^{1-\delta n-10 \delta}, \\
\left.\left|r_{m}^{2}-2 r_{m} \hat{k} \cos \left(\vartheta_{m}-\vartheta_{0}\right)+\hat{z}_{m}^{2}-a^{2}\right|<4 k^{-1+\delta n}\right\}, \\
\hat{z}_{m}=z_{m}-a .
\end{gathered}
$$

From the last equality in the right part of (7.88) and (7.53) we obtain

$$
2 r_{m}\left(k^{\prime}-\hat{k} \cos \left(\vartheta_{m}-\vartheta_{0}\right)\right)=\rho_{m q}\left(2 k-\rho_{m q}\right)+O\left(k^{2 \delta}\right) .
$$


From this relation we easily get:

$$
r_{m}\left(1-\cos \left(\vartheta_{m}-\vartheta_{0}\right)\right)<k^{3 \delta} .
$$

Thus, for $k=2,3,4,5,6,7$

$Q_{n i}^{k} \subset\left\{m:\left|\sqrt{\left(r_{m}-k^{\prime}\right)^{2}+z_{m}^{\prime}{ }^{2}}-k\right|<k^{2 \delta}, \exists \vartheta_{0}: r_{m}\left(1-\cos \left(\vartheta_{m}-\vartheta_{0}\right)\right)<k^{3 \delta}\right.$,

$$
\left.\left|\gamma_{1}\left(r_{m} \cos \vartheta_{m}-\hat{k} \cos \vartheta_{0}\right)+\gamma_{2} \hat{z}_{m}\right|<2 k^{1-\delta n-10 \delta}\right\}
$$

From inequality (7.90) we obtain that $\left|\cos \vartheta_{m}-\cos \vartheta_{0}\right|<2 k^{2 \delta} r^{-1 / 2}$. Thus, we arrive at (7.82). If $y=(z, r, \vartheta)$ is in the $A$-neighborhood of $\vec{p}_{m}(0)$, $m \in Q_{n i}^{k}, k=2,3,4,5,6,7$, then

$$
\begin{gathered}
\left|r_{m}-r\right| \leq A, \\
\left|z_{m}-z\right| \leq A, \\
\left|\cos \vartheta_{m}-\cos \vartheta\right|<2 A / r .
\end{gathered}
$$

It is clear now that formula for $\tilde{Q}_{n i}^{k}$ (A-neighborhood of $Q_{n i}^{k}$ ) coincides with formula (7.82) with respect to constant factors, namely, (7.83) holds.

\subsection{Appendix 5.}

We prove that

$$
V\left(\tilde{Q}_{n i}^{2}\right)<k^{1+3 \delta}\left(k^{1-n \delta-10 \delta}+k^{1 / 2+3 \delta}\right) .
$$

Indeed, from (7.83), taking into account that $2 r>k^{1-6 \delta}$ when $y \in \tilde{Q}_{n i}^{2}$, we obtain

$$
\tilde{Q}_{n i}^{2} \subset\left\{y: 0 \leq \vartheta \leq 2 \pi,(r, z) \in M_{\vartheta}\right\},
$$

where $M_{\vartheta}$ is the intersection of a plane layer and a ring in the plane of the variables $r$ and $z$ :

$$
\begin{gathered}
M_{\vartheta}=\left\{r, z:\left|\sqrt{\left(k^{\prime}-r\right)^{2}+{z^{\prime}}^{2}}-k\right|<2 k^{2 \delta},\left|\gamma_{1}(r-k) \cos \vartheta+\gamma_{2} z\right|<h\right\}, \\
h \equiv 2 k^{1-n \delta-10 \delta}+2 k^{1 / 2+4 \delta}
\end{gathered}
$$


It is clear that

$$
V\left(\tilde{Q}_{n i}^{2}\right) \leq \int_{0}^{2 \pi} d \vartheta \int_{M_{\vartheta}} r d r d z \leq k \int_{0}^{2 \pi} s\left(M_{\vartheta}\right) d \vartheta
$$

where $s\left(M_{\vartheta}\right)<c k^{2 \delta} \min \left\{h \hat{a}^{-1}, 3 k\right\}$. Taking into account the obvious inequality

$$
\min \left\{h \hat{a}^{-1}, 3 k\right\}<2 h\left(\hat{a}+k^{-1}\right)^{-1}
$$

we obtain

$$
s\left(M_{\vartheta}\right)<\frac{c k^{2 \delta}\left(k^{1-n \delta-10 \delta}+k^{1 / 2+4 \delta}\right)}{\sqrt{\gamma_{1}^{2} \cos ^{2} \vartheta+\gamma_{2}^{2}}+k^{-1}} .
$$

Using estimate (7.97) in the inequality (7.96) and integrating with respect to $\vartheta$ yields formula (7.93).

\subsection{Appendix 6 .}

We prove the estimate:

$$
I_{3} \leq 3 k^{1 / 2-n \delta-9 \delta}+4 k^{2 \delta}
$$

Suppose $\vec{p}_{i}(0)$ and $\vec{p}_{q}(0)$ are linearly independent. As in Appendix 4 we use the cylindrical coordinates $z, r, \vartheta$, where $z$ is directed alone $\vec{p}_{q}(0)$, and angle $\vartheta$ is assumed to be zero on the projection of the vector $\vec{p}_{i}(0)$ on the plane $z=0$. In these coordinates

$$
\begin{gathered}
\vec{p}_{i}(0)=\left(\gamma_{2}, \gamma_{1}, 0\right), \\
x_{0}=\left(p_{q}(0) / 2, k^{\prime}, \vartheta_{0}\right), x_{0} \in S_{0}
\end{gathered}
$$

where $k^{\prime}$ is given by $(7.51), \gamma_{2}$ is the projection of the vector $\vec{p}_{i}(0)$ onto $\vec{p}_{q}(0)$ and $\gamma_{1}=\sqrt{p_{i}^{2}(0)-\gamma_{2}^{2}}$. Using formula (6.37) for the linearly independent vectors $\vec{p}_{i}(0), \vec{p}_{q}(0)$, it is easy to show that $\left|\gamma_{1}\right|>k^{-\delta}$. We represent $I_{3}$ in the form $I_{3}=I_{3}^{\prime}+I_{3}^{\prime \prime}, I_{3}^{\prime}$ being the integral over the region $r>k^{3 \delta}$. Let us consider $I_{3}^{\prime}$. Taking into account that $|r|<2 k^{1-6 \delta}$ and $\rho<2 k^{2 \delta}$, from the relation (6.76) we obtain the estimates:

$$
z=\sqrt{\left(2 k^{\prime}-r\right) r}+O\left(k^{1 / 2+\delta} r^{-1 / 2}\right)
$$

$$
2 z>\sqrt{\left(2 k^{\prime}-r\right) r}>k^{1 / 2} r^{1 / 2} .
$$


Considering (7.100) and the last estimate in (7.83) and taking into account that $\left|\gamma_{2} / \gamma_{1}^{-1}\right|<k^{\delta}$, we verify that

$$
\begin{gathered}
\left|\cos \vartheta-\xi_{0}(r)\right|<\xi_{1}(r), \\
\xi_{0}(r)=\gamma_{2} \gamma_{1}^{-1} \sqrt{(2 k-r) r}(k-r)^{-1}=O\left(k^{-2 \delta}\right), \\
\gamma_{1} \xi_{1}(r)=2 k^{-n \delta-10 \delta}+4 k^{\delta} r^{-1 / 2}
\end{gathered}
$$

Producing in the integral $I_{3}$ the change of the variables $z \rightarrow \rho$ (see (7.53)), we get

$$
I_{3}^{\prime} \leq k \int_{\tilde{Q}_{n i}^{3}} \frac{r d r d \rho d \vartheta}{z(r, \rho, \varphi)\left(r^{2}+z^{2}\right)^{3 / 4}}
$$

Taking into account estimate (7.101) we show that

$$
I_{3}^{\prime} \leq k^{-1 / 4} \int_{\tilde{Q}_{n i}^{3}} r^{-1 / 4} d r d \rho d \vartheta
$$

Integrating with respect to $\rho$ and $\vartheta$ (see (7.102)), we obtain:

$$
I_{3}^{\prime} \leq k^{-1 / 4+\delta} \int_{0}^{k} r^{-1 / 4} \xi_{1}(r) d r .
$$

Evaluating the integral we get:

$$
I_{3}^{\prime} \leq 2 k^{1 / 2-n \delta-9 \delta}+4 k^{2 \delta} .
$$

Next, we consider $I_{3}^{\prime \prime}$. It is not hard to show that $z^{2}<2 k^{1+3 \delta}$ (see (7.53)), when $r<k^{3 \delta}$. Taking into account that $2 \sqrt{r^{2}+z^{2}}>k^{2 \delta}$, when $(r, z, \vartheta) \in$ $\tilde{Q}_{n i}^{3}$, we obtain $I_{3}^{\prime \prime}<k^{1 / 2+7 \delta}$. Therefore, $(7.98)$ holds when $\vec{p}_{i}(0), \vec{p}_{q}(0)$ are linearly independent, i.e., $\gamma_{1} \neq 0$.

If $\gamma_{1}=0$, then it follows from formula (7.88) that

$$
Q_{n i}^{3} \subset\left\{m:\left|\sqrt{\left(r_{m}-k^{\prime}\right)^{2}+z_{m}^{\prime 2}}-k\right|<k^{2 \delta},\left|z_{m}\right|<k^{1-\delta n-10 \delta}\right\} .
$$

From this we obtain:

$$
\tilde{Q}_{n i}^{3} \subset\left\{y:\left|\sqrt{\left(r-k^{\prime}\right)^{2}+z^{2}}-k\right|<2 k^{2 \delta},|z|<2 k^{1-\delta n-10 \delta}\right\} .
$$


It is not hard to show that

$$
\tilde{Q}_{n i}^{3} \subset\left\{y:|z|<2 k^{1-\delta n-10 \delta},\left|r-r_{0}(z)\right|<k^{2 \delta}\right\},
$$

$r_{0}(z)$ being uniquely determined from the relations $z^{2}=r_{0}\left(2 k-r_{0}\right), r_{0}<$ $k^{1-3 \delta}$. We use again formula (6.81). To estimate $I_{3}$ we again represent it as the sum $I_{3}^{\prime}+I_{3}^{\prime \prime}$. Using (7.101), we get

$$
I_{3}^{\prime} \leq k^{-3 / 4} \int r^{1 / 4} d r d z
$$

Taking into account (7.108), it is not hard to check estimate (7.106). The integral $I_{3}^{\prime \prime}$ we estimate as before. Thus, inequality (7.98) holds for linearly dependent $\vec{p}_{i}(0), \vec{p}_{q}(0)$ too.

\subsection{Appendix 7.}

We prove that

$$
I_{4}<k^{7 / 4-\delta n-6 \delta}+k^{1+2 \delta} .
$$

From the relations $4 k^{2}-r_{m}^{2}-z_{m}^{2}>k^{1+3 \delta}, \rho<k^{2 \delta}$ and (7.53) it easily follows that $2 k-r>(1 / 4) k^{3 \delta}$. Taking into account that $2 k-r<k^{1-3 \delta}$ we obtain from (6.76)

$$
\begin{gathered}
|z|=\sqrt{(2 k-r) r}+O\left(k^{1 / 2+2 \delta}(2 k-r)^{-1 / 2}\right), \\
2|z|>k^{1 / 2}(2 k-r)^{1 / 2}, \\
4 k^{2}-r^{2}-z^{2}>k(2 k-r) .
\end{gathered}
$$

Suppose $\vec{p}_{i}(0)$ and $\vec{p}_{q}(0)$ are linearly independent. As in Appendix 4 we used the cylindrical coordinates $z, r, \vartheta$, where $z$ is directed alone $\vec{p}_{q}(0)$, and angle $\vartheta$ is assumed to be zero on the projection of the vector $\vec{p}_{i}(0)$ on the plane $z=0$. In these coordinates

$$
\begin{gathered}
\vec{p}_{i}(0)=\left(\gamma_{2}, \gamma_{1}, 0\right), \\
x_{0}=\left(p_{q}(0) / 2, k^{\prime}, \vartheta_{0}\right), x_{0} \in S_{0},
\end{gathered}
$$

where $k^{\prime}$ is given by (7.51), $\gamma_{2}$ is the projection of the vector $\vec{p}_{i}(0)$ onto $\vec{p}_{q}(0)$ and $\gamma_{1}=\sqrt{p_{i}^{2}(0)-\gamma_{2}^{2}}$. Using formula (6.37) for the linearly independent 
vectors $\vec{p}_{i}(0), \vec{p}_{q}(0)$, it is easy to show that $\left|\gamma_{1}\right|>k^{-\delta}$ Considering estimate (7.111), the second inequality on the right of (7.83) and the relation $2 k-r>k^{2 \delta}$, we verify that

$$
\begin{gathered}
\left|\cos \vartheta-\xi_{0}(r)\right|<\xi_{2}, \\
\gamma_{1} \xi_{2}=2 k^{-n \delta-2 \delta}+2 k^{-1 / 2+\delta},
\end{gathered}
$$

$\xi_{0}$ being given by (7.102). Producing in the integral $I_{4}$ the change of the variables $z \rightarrow \rho$, and using estimate (7.113) we obtain:

$$
I_{4} \leq k^{5 / 4+\delta} \int_{\tilde{Q}_{n i}^{4}}(2 k-r)^{-3 / 4} d r d \rho d \vartheta .
$$

Integrating with respect to $\rho\left(0<\rho<k^{\delta}\right), \vartheta$ (see (7.115)) and $r, 0 \leq r \leq 2 k$, we arrive at the inequality: $I_{4}<k^{3 / 2+\delta} \xi_{2}$. Using (7.116), we get

$$
I_{4}<k^{3 / 2-n \delta-\delta}+k^{1+2 \delta}, \quad, \gamma_{1} \neq 0 .
$$

Suppose $\gamma_{1}=0$. Using (7.111), from relation (7.108) we obtain

$$
\tilde{Q}_{n i}^{k} \subset\left\{y:|z|<2 k^{1-\delta n-10 \delta},\left|r-r_{0}(z)\right|<k^{\delta}\right\},
$$

where $r_{0}$ is uniquely determined from the relations $k^{3 \delta}<2 k-r_{0}<k^{1-3 \delta}$, $r_{0}\left(2 k-r_{0}\right)=z^{2}$. Now, from relations (6.87) and (7.113), we get:

$$
I_{4}<k^{3 / 4} \int_{\tilde{Q}_{n i}^{4}}(2 k-r)^{-1 / 4} d r d z d \vartheta<k^{7 / 4-\delta n-6 \delta}, \gamma_{1}=0 .
$$

Estimates (7.118), (7.119) together give (7.110).

\subsection{Appendix 8.}

We prove that

$$
V\left(\tilde{Q}_{n i}^{5}\right)<k^{7 / 4-\delta n-6 \delta}+k^{1+2 \delta} .
$$

It is not hard to show that the formula for $\tilde{Q}_{n i}^{5}$ is similar to that of $\tilde{Q}_{n i}^{5}$. It is clear that

$$
V\left(\tilde{Q}_{n i}^{5}\right)=\int_{\tilde{Q}_{n i}^{5}} r d r d z d \vartheta
$$


We use formula (7.83) for $\tilde{Q}_{n i}^{5}$. Considering as above (see (7.115)), we show that $\left|\cos \vartheta-\xi_{0}\right|<\xi_{2}$. From the relation $4 k^{2}-r^{2}-z^{2}<k^{1+3 \delta}$ and (6.91) it follows that $z^{2}<k^{1+3 \delta}$. Using (6.90), we obtain $2 k-r_{m}=O\left(k^{3 \delta}\right)$. Considering this estimate in the formula (7.102) for $\xi_{0}$ we show that , $\xi_{0}=$ $o(1)$. Therefore, $V\left(\tilde{Q}_{n i}^{5}\right)<c k^{3 / 2+5 \delta} \xi_{2}$ and (7.120) holds.

\subsection{Appendix 9.}

We estimate each term of the sum (6.117) and obtain the estimate

$$
s\left(\hat{\Upsilon}_{q}(k, V, \delta)_{4 q^{\prime}}\right)<c p_{q}^{-1}(0) k^{1-4 \delta},
$$

Using the estimate similar to (6.28) and considering as in the proof of Lemma 11 , we verify that

$$
\begin{gathered}
\Sigma^{1}<c k^{-1 / 5-8 \delta} p_{q}^{-1}(0) I_{1}, \\
I_{1}=\int_{\tilde{Q}}(\rho(y) r(y))^{-1 / 2} r d r d z d \vartheta,
\end{gathered}
$$

$\tilde{Q}$ being $A$-neighbourhood of $Q$. Note that

$$
2 k \rho-\rho^{2}=2 k^{\prime} r-r^{2}-z^{2}+O\left(k^{4 \delta}\right) .
$$

Taking into account that $r \geq \rho>k^{2 \delta},|z|<2 k^{1 / 5}$ (see (6.116)), we obtain $\rho>r / 2$ for $y \in \tilde{Q}$. Therefore,

$$
I_{1}<\int_{\tilde{Q}} d r d z d \vartheta<c k^{6 / 5}
$$

Using this estimate in (7.122), we obtain

$$
\Sigma^{1}<c p_{q}^{-1}(0) k^{1-4 \delta} \text {. }
$$

It is not hard to show, using (6.116) and (7.123) that $Q^{2}=\emptyset$. Therefore, $\Sigma^{2}=0$.

We consider $\Sigma^{3}$. Since $m \in Q^{3}$, then estimate (6.47) is valid. From relation (7.123), taking into account that $\rho_{m q}<k^{2 \delta}$, we obtain

$$
p_{m}^{2}(0)=r_{m}^{2}+z_{m}^{2}=2 r_{m} k+O\left(k^{1+2 \delta}\right) .
$$

Taking into account this inequality, and considering that $r_{m}<k^{1-\delta}$, $|z|<2 k^{1 / 5}$, we obtain $4 k^{2}-p_{m}^{2}(0)>k^{2}$. Using the last estimate in inequality (6.47), we get

$$
s\left(\hat{S}_{q}(k,-\delta) \cap \Pi_{m}^{\prime}(k, 1 / 5+8 \delta)\right)<c k^{4 / 5-7 \delta} p_{q}^{-1}(0) p_{m}^{-3 / 2}(0) .
$$


Substituting estimate (7.126) in formula for $\Sigma^{3}$, we obtain:

$$
\Sigma^{3} \leq c k^{4 / 5-7 \delta} p_{q}^{-1}(0) \sum_{m \in Q^{3}} p_{m}(0)^{-3 / 2} .
$$

Since $p_{m}(0)>k^{2 \delta}$, when $m \in Q^{3}$, the series on the right of estimate (7.127) can be estimated by the integral

$$
\begin{gathered}
\Sigma^{3} \leq c k^{4 / 5-7 \delta} p_{q}^{-1}(0) I_{3} . \\
I_{3}=\int_{\tilde{Q}_{n i}^{3}} r\left(r^{2}+z^{2}\right)^{-3 / 4} d r d z d \vartheta .
\end{gathered}
$$

From the relations $\rho<k^{2 \delta},|z|<k^{1 / 5}$ and (7.123) it follows that $r<c k^{2 \delta}$. Using that $p_{m}(0)>k^{2 \delta}$, we obtain $I_{3}<k^{1 / 5+2 \delta}$. Therefore,

$$
\Sigma^{3}<c p_{q}^{-1}(0) k^{1-4 \delta}
$$

We estimate $\Sigma^{4}$. Since $m \in Q^{4}$, estimate (6.29) is valid. Using relations $p_{m}(0)>r_{m}>k, p_{q}(0)<k^{\delta}$, we rewrite estimate (6.29) in the form:

$$
s\left(\hat{S}_{q}(k,-\delta) \cap \hat{\Pi}_{m}(k, 1 / 5+8 \delta)\right)<c p_{q}^{-1}(0) k^{-1 / 5-7 \delta}\left(4 k^{2}-r_{m}^{2}-z_{m}^{2}\right)^{-1 / 4} .
$$

Using estimate (7.131) in formula for $\Sigma^{4}$, we obtain

$$
\Sigma^{4} \leq c p_{q}^{-1}(0) k^{-1 / 5-7 \delta} \sum_{m \in Q^{4}}\left(4 k^{2}-r_{m}^{2}-z_{m}^{2}\right)^{-1 / 4} .
$$

It is clear that $\tilde{Q}^{4}$ can be described by a formula similar to the formula for $Q^{4}$. Since $4 k^{2}-r_{m}^{2}-z_{m}^{2}>k^{1+2 \delta}$, when $m \in Q^{4}$, the series on the right of (7.132) can be estimated by the integral

$$
\Sigma^{4} \leq c p_{q}^{-1}(0) k^{-1 / 5-7 \delta} I_{4}
$$

$$
I_{4}=\int_{\tilde{Q}^{4}} \frac{r d r d z d \vartheta}{\left(4 k^{2}-r^{2}-z^{2}\right)^{1 / 4}} .
$$

From the relations $|z|<k^{1 / 5}, \rho<k^{2 \delta}$ and (7.123) it is easily follows that $2 k-r<k^{3 \delta}$. Therefore,

$$
I_{4}<c k
$$


and

$$
\Sigma^{4} \leq c p_{q}^{-1}(0) k^{1-4 \delta} .
$$

Let us consider $\Sigma^{5}$. We prove that $4 k^{2}-p_{m}^{2}(0)>k^{-1+3 \delta}$. Indeed, using (7.53), we obtain

$$
\left(2 k^{\prime}-r_{m}\right) r_{m}=z_{m}^{\prime 2}+2 \rho_{m q} k-\rho_{m q}^{2}+O\left(k^{2 \delta}\right) .
$$

Therefore,

$4 k^{2}-r_{m}^{2}-z_{m}^{2}=\left(z_{m}^{\prime 2}+2 \rho_{m q} k-\rho_{m q}^{2}+O\left(k^{2 \delta}\right)\right)\left(2 k+r_{m}\right) r_{m}^{-1}-z_{m}^{2}>z_{m}^{2}>k^{4 \delta}$.

From this $2 k-p_{m}(0)>k^{-1+3 \delta}$. Thus, estimate (6.29) is valid. Using relations $p_{m}(0)>r_{m}>k, p_{q}(0)<k^{\delta},(6.91)$ and (7.135) we obtain

$$
\Sigma^{5} \leq k^{-1 / 5-7 \delta} p_{q}^{-1}(0) \sum_{Q^{5}} 1 .
$$

It is clear that

$$
\Sigma^{5} \leq k^{-1 / 5-7 \delta} p_{q}^{-1}(0) V\left(\tilde{Q}^{5}\right),
$$

$\tilde{Q}^{5}$ being the $A$-neighbourhood of $Q^{5}$. Obviously, the formula for $\tilde{Q}^{5}$ is similar to that for $\tilde{Q}^{5}$ and

$$
V\left(\tilde{Q}^{5}\right)=\int_{\tilde{Q}^{5}} r d r d z d \vartheta
$$

From the relation $4 k^{2}-r^{2}-z^{2}<k^{1+3 \delta}$ (see (6.123)) and (7.53) it follows that

$$
2 k-r=-\rho+\rho^{2} / 2 k+O\left(k^{3 \delta}\right)=O\left(k^{3 \delta}\right) .
$$

Taking into account that $|z|<k^{1 / 5}$, we obtain

$$
V\left(\tilde{Q}^{5}\right)<c k^{6 / 5+3 \delta} .
$$

Using this estimate in (7.138), we get

$$
\Sigma^{5}<c p_{q}^{-1}(0) k^{1-4 \delta} .
$$

The sums $\Sigma_{6}$ and $\Sigma_{7}$ can be estimated just as $\Sigma_{i n}^{6}, \Sigma_{i n}^{7}$ in Lemma 11. Adding the inequalities for $\Sigma^{k}$ we get (7.121). 
The author expresses deep gratitude to B.S. Pavlov for useful discussions of this work.

\section{References.}

[1] Yu. E. Karpeshina Perturbation theory for the Schrödinger operator with a periodic potential Trudy Mat. Inst. Steklov. 188 (1990); English transl., Proc. Steklov Inst. Math. 1991, no. 3(188), 109-145.

[2] Yu. E. Karpeshina Perturbation theory formulas for the Scrödinger equation with a nonsmooth periodic potential. Matem. Sbornik, 181, 9; English transl. Math. USSR Sbornik, 71(1992),1, 101-122. 66 (1991) 557-579.

[3] I.M. Gel'fand, Expansion in eigenfunctions of an equation with periodic coefficients, Dokl. Akad. Nauk SSSR, 73 (1950),1117-1120. (Russian)

[4] O. Madelung Festkörpertheorie, Vols. I, II, Springer-Verlag, 1972: English transl., 1978.

[5] J. Feldman, H. Knörrer, E. Trubowitz, Perturbatively unstable eigenvalues of a periodic Schrödinger operator. Comment. Math. Helvetici

[6] O.A. Veliev Review by the author of the doctoral thesis. Tbilisi-1989 (in Russian).

[7] Yu. E. Karpeshina, Analytic perturbation theory for a periodic potential, Izv. Akad. Nauk SSSR Ser. Mat. 53 (1989), 45-65; English transl. in Math. USSR Izv. 34 (1990)

[8] Yu.E. Karpeshina Perturbation series for polyharmonic operator with a periodic potential near planes of diffraction. Preprint, ETH, Zürich, 1992.

[9] Yu.E. Karpeshina Perturbation formulae for the Schrödinger operator with a periodic potential near planes of diffraction, Mathematical Research Letters, 2, $59-74$ (1995).

ReCEIVEd JANuARY 16, 1996 AND REvised June 7, 1996.

UNIVERSiTy of ALABAMA

Campbell Hall

1330 UNIV. BLVD.

BiRMINGHAM, AL 35294-1170

E-MAIL:KARPESHI@VORTEB.MATH.UAB.EDU 